\title{
Analysis of multiclass Markovian polling systems with feedback and composite scheduling algorithms
}

\author{
Tetsuji Hirayama
}

\begin{abstract}
We consider multiclass Markovian polling systems with feedback and analyze their average performance measures. Scheduling in polling systems has many applications in computer and communication systems. We utilize the framework that has been effectively used to analyze various composite scheduling algorithms in many types of multiclass queues systematically in conjunction with the functional computation method (Hirayama, 2003, 2005, 2009a, 2010).

We define the conditional expected values of the performance measures such as the sojourn times as functions of the system state and find their expressions by solving some equations. Then from these expressions, we derive the average numbers of customers and the average sojourn times for all service stages of customers circulating the system. We consider their application to a packet scheduling problem where multiple categories of packets share a resource.
\end{abstract}

Keywords Multiclass queues · Feedback · Markovian polling · Packet scheduling

\section{Introduction}

Multiclass queueing systems have been extensively studied to analyze packet scheduling problems in computer communication networks (Kleinrock, 1976). These systems have multiple classes of customers with various features in quantities such as arrival rates, service times, feedback probabilities and service paths, and have many kinds of scheduling algorithms such as priorities and server allocation policies. They are powerful tools for performance evaluation because they can analyze detailed structures of communication networks with many types of application packets and scheduling algorithms. Our aim is to develop the method of analyzing full-scale multiclass queueing models that can investigate real packet scheduling problems in detail.

Faculty of Engineering, Information and Systems, University of Tsukuba Tsukuba-shi, Ibaraki 305-8573, JAPAN

Tel.: +81-29-853-5325

Fax: +81-29-853-5206

E-mail: hirayama@cs.tsukuba.ac.jp

The formal version of this paper was published in Annals of Operations Research, 198, 83-123, (2012). 
We consider a two level classification of customers where there are multiple groups (stations) each of which comprises multiple classes of customers. A single server visits a group at a time, and then some customers in the group are admitted into the service facility and are served according to some service order that utilizes their classes. Hence the scheduling algorithms for the multiclass queues are effectively prescribed by the following items.

1. Selection (or visiting) orders of groups by the server.

2. Customer selection rules used when the server admits customers in each group into the service facility.

3. Service orders of customers in the service facility.

Typical selection orders of groups are a priority order and a (cyclic) polling order. There are many types of customer selection rules, e.g., gated, exhaustive and 1-limited. There are also many service orders of customers, e.g., FCFS, priority, PS (processor sharing), LAS (least attained service first), SJF (shortest job first), etc. The DRR (discriminatory round robin) considered in (Hirayama, 2010) is another example. (Note that not all of these combinations can be analyzed by our method.)

This framework for scheduling algorithms has been effectively used to analyze various composite scheduling algorithms in many types of multiclass queues systematically in conjunction with the functional computation method ((Hirayama, 2003, 2010) for the multiclass priority systems with feedback, (Hirayama, 2005) for the multiclass cyclic polling systems with feedback). We also use this framework to analyze multiclass Markovian polling systems with feedback in this paper. A similar framework was considered later in (Wierman et al., 2007; Boxma et al., 2009) to analyze scheduling in polling systems. Because in our models we treat only the average values of the performance measures, e.g., the average numbers of customers and the average sojourn times, we often do not explicitly mention whether or not distributions of performance measures were considered in the existing models taken up in this section.

Priority order is a major one of the selection orders of groups. Many researchers have investigated multiclass queueing systems with priority. Kleinrock (1976) extensively investigated the systems and their applications to packet scheduling problems. These multiclass systems have been extended to the systems with feedback where customers can receive multiple services by returning to the systems several times. The Bernoulli feedback systems with multiple groups considered in (Choi et al., 2000; Doshi and Kaufman, 1988) are these systems where service times, feedback probabilities, and the other statistical quantities of customers in each group, respectively, are independent and identically distributed at all of their service stages. In the systems (Jewkes and Buzacott, 1991; Van den Berg et al., 1989), statistical quantities and priorities at all service stages of customers can be different, but all customers essentially belong to a single group.

The feedback systems in (Paterok et al., 1989; Simon, 1984) have multiple groups with the 1-limited customer selection rule where statistical quantities and priorities at all service stages of customers in every group can be different. These systems were extended to the Markovian feedback system (Hirayama, 2003) that has multiple classes in each group with the gated rule as a customer selection rule and with FCFS order and priority order as service orders of customers in the service facility. In (Hirayama, 2010) the system was further extended to the system with mixtures of the 1-limited, the gated, and the exhaustive customer selection rules. 
The other major selection order of groups is (cyclic) polling order. The cyclic polling systems with a single class in each group were considered in many papers ((Cooper, 1970; Cooper and Murray, 1969) for the systems with zero switchover periods, (Eisenberg, 1972) for the system with nonzero switchover periods). Although these papers utilized the buffer occupancy method (BOM), which has been the major method for analyzing the polling systems, many other methods have also been investigated. The station time method (STM) (Ferguson and Aminetzah, 1985), the functional computation method (FCM) (Hirayama et al., 2004), and the mean value analysis (MVA) (Winands et al., 2006) have been developed. The descendant set method which is a variation of BOM was considered in (Konheim et al., 1994), and was used to analyze polling systems with simultaneous batch arrivals in (Van der Mei, 2002). Another method was also considered in (Sarkar and Zangwill, 1989). These variations and the methods of analysis were surveyed in (Levy and Sidi, 1990; Takagi, 1986).

The cyclic polling systems have also been extended to the systems with feedback. The symmetric polling system with Bernoulli feedback was analyzed by the stochastic decomposition property in (Takine et al., 1991). The two-stage tandem system with multiple customer groups discussed in (Katayama, 1992), which was analyzed by the standard embedded Markov chain approach, is a variation of the cyclic polling system with feedback. Sidi et al. (1992) analyzed by BOM the cyclic polling system with Markovian feedback that has a single class in each group. Hirayama (2005) extended it to the system that has multiple classes in each group with the gated or the exhaustive rule as customer selection rules and with FCFS or priority order as service orders of customers in the service facility, and analyzed the system by FCM.

Although the cyclic polling order is a typical selection order of groups by the server, we can consider the other polling orders. A system with a general deterministic order was investigated by STM in (Baker and Rubin, 1987). A system with a random order (Kleinrock and Levy, 1988), and a system with a general probabilistic order (Srinivasan, 1991) were analyzed by BOM. Hirayama (2009a) investigated a system with a Markovian order by FCM, and calculated its computational complexity. In this paper we further extend it to the system with Markovian feedback that has multiple classes in each group with the gated or the exhaustive rule as a customer selection rule and with FCFS or priority order as a service order in the service facility. Such general polling orders can give preferential treatments to some groups of customers by appropriately arranging the frequencies that the server visits them. In Section 7, we give such examples in a packet scheduling problem. To the best of our knowledge, this Markovian polling system with feedback and with the two level classification of customers has not been investigated.

Concerning customer selection rules, the gated rule and the exhaustive rule have commonly been used in many of the papers. One of the other rules, the 1-limited rule, is fairly difficult to analyze except for some special cases (Levy and Sidi, 1990). The symmetric polling system with the 1-limited rule and Bernoulli feedback was analyzed in (Takine et al., 1991). The other (analyzable) variations, the binomial gated rule and the globally gated rule, were investigated by BOM in (Levy, 1991) and by the cycle time analysis in (Boxma et al., 1992), respectively. Strictly speaking, because the globally gated rule is outside the above framework, we somewhat need to modify it in order to treat the rule.

The only service order of customers (in the service facility) in the polling systems has been FCFS for a long time, because there has been a single class in each group. Recently several service orders in each group with multiple classes of customers have 
been considered. The mean sojourn times in the cyclic polling system with the gated and the exhaustive groups that have several service orders including priority, PS and SJF were analyzed by MVA in (Wierman et al., 2007). The LSTs of the sojourn time distributions in the cyclic polling system with either the gated or the globally gated rule and with several service orders including PS and SJF were obtained in (Boxma et al., 2009) by combining the method developed by Resing (1993) for branching type selection rules and the cycle time analysis. Further the LSTs of the sojourn time distributions in the cyclic polling system with either the gated, the exhaustive or the globally gated rule in which customers in each group are served in a priority order were obtained in (Boon et al., 2010) by combining the above Resing's method, the cycle time analysis and the decomposition property. The average sojourn times in the cyclic polling system with feedback that has the gated and the exhaustive rules and has FCFS and priority service orders were obtained in (Hirayama, 2005) by FCM. In this paper we extend it to Markovian polling systems.

PS and LAS are "infinitesimal quanta algorithms" where all relevant customers are served simultaneously by dividing their service times into infinite numbers of infinitesimal service quanta. In contrast to these algorithms, we can consider "positive quanta algorithms" where a service time of each customer is divided into a finite number of positive service quanta and where all relevant customers receive their quanta one after another until they complete their services. We can model these positive quanta algorithms by the feedback systems. DRR considered in (Hirayama, 2010) is a positive quanta version of DPS (discriminatory processor sharing) (Altman et al., 2006) which is a multiclass extension of PS. DPS is used to approximate the actual packet scheduling algorithms like WFQ (weighted fair queue) (Aalto et al., 2007). In (Hirayama, 2010) DRR was combined with the priority selection order to approximate the actual scheduling algorithms like LLQ (low latency queue) and IP-RTP priority that combine WFQ with PQ (priority queue). We can also combine DRR with the polling selection orders.

From the perspective of the mathematical analysis, we use the functional computation method (FCM) which has been developed in order to analyze performance of many M/G/1 type multiclass queues and various scheduling algorithms. This method was used to analyze the polling system (Hirayama et al., 2004) and its extension to the multiclass polling system with feedback (Hirayama, 2005). Further it was used to analyze the multiclass feedback queue with priority order and the gated selection rule (Hirayama, 2003) and its extension to the system with mixtures of the 1-limited, the gated and the exhaustive selection rules (Hirayama, 2010).

The main differences between the classical methods (e.g. BOM and STM) and FCM are as follows. These classical methods analyze evolution of the system states along time and derive the equations satisfied by their moments (or transforms of their distributions). The system states of BOM are numbers of customers at polling instants, and those of STM are terminal service times each of which consists of a service period and a switchover period. Then the average waiting times can be derived by relating them to these moments of the system states. On the other hand, the analysis of FCM is accomplished as follows. By observing any one of customers, its expected sojourn times conditioned on its arrival epochs (or its related polling instants) are derived stepby-step (often by solving equations). Then the average sojourn times are derived by averaging those conditional expected sojourn times of all customers, and the average number of customers are derived by the Little's formula and the PASTA property. The relationship between BOM and FCM was given in (Hirayama et al., 2004; Hirayama, 
2005). The other difference is their ranges of analysis. These classical methods can only analyze polling systems while FCM can analyze not only polling systems but also priority systems and feedback systems (as described above).

The whole analysis of a queueing system by FCM is carried out by adequately combining analysis common to various systems and analysis intrinsic to the individual system. First the states of the system are defined, and then its performance measures such as the conditional expected sojourn times are defined as functions of the states. The performance measures and their components are related by some equations (e.g. the feedback equations and the polling equations). Expressions for these components are derived by analyses intrinsic to the system, whereas expressions for the performance measures are derived from these equations whose solution methods are common to various systems. Further steady state values of the performance measures are obtained from these expressions by applying some common limiting procedures, the Little's theorem and the PASTA property.

These common parts of the analysis give the excellent characteristics that our method can analyze various systems. But when we investigate any individual system, we should use its individual structures in order to give its concrete model description such as the system states and the performance measures, and in order to obtain their expressions and their steady state values in detail. For example, the analysis of busy periods and delay cycles is used to analyze the priority systems (Hirayama, 2003, 2010), whereas the analysis of numbers of customers at polling instants is used to analyze the polling systems (Hirayama, 2005; Hirayama et al., 2004). It is the linear functional expressions that closely link the common parts with the individual parts.

To see these excellent characteristics, we can compare the expressions in equations (5.12) and (5.13) in (Hirayama, 2005) with the expressions in equations (60) and (61) in (Hirayama, 2010). These expressions are essentially the same except for those related to the switchover periods $\left(k \in \Pi^{s}\right.$ in (5.12)) despite the considerable structural differences between the former polling system and the latter priority system. However the derivation procedures of the expressions for their coefficients $\left(\boldsymbol{\varphi}_{i, \alpha}(\cdot), \mathbf{w}_{i, \alpha}(\cdot), w_{i, \alpha}(\cdot)\right.$, etc.) in these two systems are of course fairly different. Another advantage of the method is that similar analysis can be applied to both of the systems with switchover times and without switchover times.

The paper is organized as follows. In Section 2 we describe our model of the multiclass Markovian polling systems with feedback. We define the system structures and the scheduling algorithms in detail along the framework given above. Then we define the system states and the conditional expectations of the performance measures. In Section 3 we analyze service periods of every station for all scheduling algorithms. In Section 4 the components of the expected performance measures and their related quantities at every service stage of a tagged customer are analyzed. In Section 5 we obtain the linear functional expressions for the conditional expectations of the performance measures. Then we consider their steady state average values in Section 6 . We consider an application to a packet scheduling problem where three categories of packets share a resource in Section 7 . We construct the four scheduling algorithms and compare their performance. The algorithm for calculating the performance measures and its computational complexity are given in the appendices. 


\section{Model Description}

In this section, we describe our model of the multiclass queues.

\subsection{The system structures and the parameters}

There are $J$ groups of customers and $L_{i}$ classes of customers in group $i$. $(i, \alpha)$-customers (belonging to class $\alpha$ in group $i$ ) arrive at station $i$ from outside the system according to a Poisson process with rate $\lambda_{i, \alpha}$. Let $\mathcal{S}=\left\{(i, \alpha): i=1, \ldots, J\right.$ and $\left.\alpha=1, \ldots, L_{i}\right\}$, $J_{c}=\sum_{i=1}^{J} L_{i}$, and $\lambda=\sum_{i=1}^{J} \sum_{\alpha=1}^{L_{i}} \lambda_{i, \alpha}$. All customers, whose arrival rate is $\lambda$, are numbered in order of arrival where $\mathbf{c}^{e}$ and $\tau_{0,0}^{e}$ denote the $e^{\text {th }}$ arriving customer itself and its arrival epoch, respectively $(e=1,2, \ldots)$. (We use $\tau_{l, \ell}^{e}(l, \ell=0,1,2, \ldots)$ to denote the typical time epochs related to $\mathbf{c}^{e}$, whose definitions are given below. Also see Fig.1.)

Each arriving customer receives service many times while changing its group and class. A service stage is a time period from when a customer arrives at a station until it completes a service at the station. Let $S_{i, \alpha}$ be a service time an $(i, \alpha)$-customer receives during a service stage whose mean and second moment are $E\left[S_{i, \alpha}\right]>0$ and $\overline{s_{i, \alpha}^{2}}$, respectively. After completing a service, an $(i, \alpha)$-customer either returns to the system as a $(j, \beta)$-customer with probability $p_{i, \alpha, j, \beta}$ or departs from the system. The feedback probability matrix is given by $\mathbf{P}=\left(p_{i, \alpha, j, \beta}:(i, \alpha),(j, \beta) \in \mathcal{S}\right)$. We assume that $\mathbf{P}^{n} \rightarrow \mathbf{O}$ as $n \rightarrow \infty$. Then let $T_{i, \alpha}$ be a total amount of service times received by a customer from when it becomes an $(i, \alpha)$-customer until it departs from the system. Its expected value $\bar{T}_{i, \alpha}$ satisfies the following equation.

$$
\bar{T}_{i, \alpha}=E\left[S_{i, \alpha}\right]+\sum_{k=1}^{J} \sum_{\gamma=1}^{L_{k}} p_{i, \alpha, k, \gamma} \bar{T}_{k, \gamma}, \quad(i, \alpha) \in \mathcal{S} .
$$

Then, let $\rho=\sum_{i=1}^{J} \sum_{\alpha=1}^{L_{i}} \lambda_{i, \alpha} \bar{T}_{i, \alpha}<1$ be the resource utilization of the system.

A single server visits these stations according to a Markovian polling order. That is, for any $i=1, \ldots, J$, when the server completes necessary services of customers at station $i$ (defined below as a period $i$ ), one of the following two cases occurs:

- If the system is not empty, the server selects station $j$ with probability $\hat{p}_{i j}$ and then visits it immediately $(j=1, \ldots, J)$. If the visiting station $j$ is not empty, the server begins services of its customers; while if it is empty, the server again selects station $k$ immediately according to the probability $\hat{p}_{j k}$ and then visits it $(k=1, \ldots, J)$, and so on. This process continues until the server reaches a non-empty station.

- If the system is empty, the server becomes idle. At the next time a customer arrives, the server immediately starts its service.

All switchover times spent when the server moves between stations are assumed to be 0 . The server cannot be idle whenever the system is not empty. Let $\hat{\mathbf{P}}=\left(\hat{p}_{i j}\right.$ : $i, j=1, \ldots, J)$ be the switching probability matrix. We assume that the Markov chain generated by the transition probability matrix $\hat{\mathbf{P}}$ is irreducible.

The system is separated into two parts, which are called the "service facility" and the "waiting room." There is a gate at each station between the set of its queues in the waiting room and the set of its queues in the service facility, which intercepts the 
migration of customers between them. Each customer arriving at each station from outside the system or by feedback enters its queue in the service facility when its gate is opened; otherwise, it enters its queue in the waiting room.

The server visits one of the stations at a time, and then opens its gate in order to admit some customers at the station to its queues in the service facility. Then, the server serves the customers in the service facility until the server empties it, and then visits another station and opens its gate. Since the gates of the stations that are not visited by the server are closed, all customers at such stations must wait for service in the waiting room. Once a customer begins a service, it is not interrupted by other customers (that is, each service in each service stage is non-preemptive).

Each time interval from when the server visits a station until the first time the server empties the service facility is called a "service period." Each time interval when the server is idle is called an "idle period." We use an abbreviated term "period $k$ " to denote a service period during which the server visits station $k$ if $k \neq 0$ or an idle period if $k=0$. Let $\Pi=\{1, \ldots, J\}$ be the set of indices of the service periods.

\subsection{The scheduling algorithms}

Customers in the system are served according to a predetermined scheduling algorithm. As we explained in Introduction, it is prescribed by (1) selection orders of the groups (stations) by the server, which is the Markovian polling as described before; (2) customer selection rules in each group, which is either the gated or the exhaustive; (3) service orders of customers in the service facility, which is either the FCFS, or the fixed priority $(F P) . \mathcal{H}_{e}, \mathcal{H}_{e F}$ and $\mathcal{H}_{e P}$ denotes the set of the groups with the exhaustive rule (the exhaustive groups), the set of this groups with the FCFS order (the exhaustive FCFS groups) and the set of this groups with the FP order (the exhaustive priority groups), respectively. $\mathcal{H}_{g}, \mathcal{H}_{g F}, \mathcal{H}_{g P}$ are similarly defined for the groups with the gated rule.

For any gated group, the gate is opened just when the server polls the group, and all customers staying in the group at this polling instant are admitted into the service facility, and then the gate is closed immediately. For any exhaustive group, the gate is opened just when the server polls the group, and it remains open and all customers staying in the group are admitted into the service facility until it is cleared of customers. For the FCFS service order, both of exogenous arrival epochs and feedback arrival epochs are considered to be the "coming" epochs which are used to decide its service order. That is, every customer arriving (exogenously or by feedback) at any group with the FCFS order joins the tail of its queue. For the FP order, customers in each group have the local nonpreemptive priority order where class $\alpha$ customers have priority over class $\beta$ customers if $\alpha<\beta$ and the order is effective only within the group.

\subsection{The system states}

Let us consider the system operating under a specified scheduling algorithm. For a while, we give attention to $\mathbf{c}^{e}$ arriving at $\tau_{0,0}^{e}(e=1,2, \ldots)$. Then let $\tau_{l, 0}^{e}$ be the time just when, after completing its $l^{\text {th }}$ service stage, it arrives (by a feedback) at one of the stations or departs from the system $(l=1,2, \ldots)$. Further let $\left\{\tau_{l, \ell}^{e}: \ell=1,2, \ldots\right\}$ be a sequence of all polling instants (i.e., service period beginning epochs) of all stations 


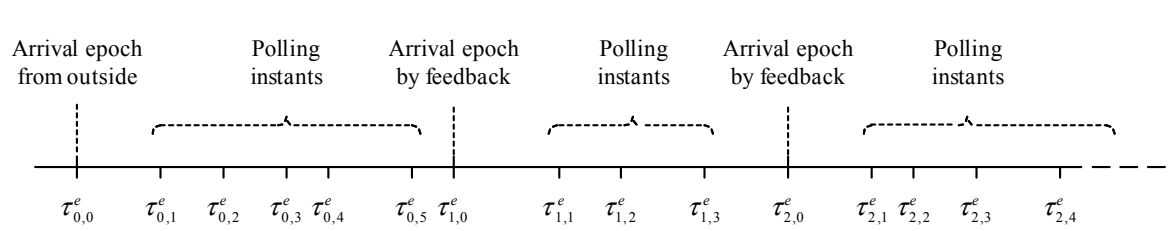

Fig. 1 Arrival epochs and polling instants related to $\mathbf{c}^{e}$

which occur after the $\mathbf{c}^{e}$ 's arrival epoch $\tau_{l, 0}^{e}$ (see Fig.1). We assume that $\tau_{l, 0}^{e} \leq \tau_{l, 1}^{e} \leq$ $\tau_{l, 2}^{e} \leq \cdots$. At $\mathbf{c}^{e}$ 's arrival epoch $\tau_{l, 0}^{e}$, if the system is empty (when $l=0$ ) or a service period ends (when $l>0$ ), then a polling instant occurs immediately and $\tau_{l, 0}^{e}=\tau_{l, 1}^{e}$.

The stochastic process with state space $\mathcal{E}$ is defined by

$$
\mathcal{Q}=\{\mathbf{Y}(t)=(\kappa(t), a(t), r(t), \mathbf{g}(t), \mathbf{n}(t), L(t)): t \geq 0\}
$$

where

- $(\kappa(t), a(t)) \in \mathcal{S} \cup\{(0,0)\}$ is the status of the server at time $t$, that is, a $(\kappa(t), a(t))$ customer is being served if $\kappa(t) \in \Pi$, or the server is idle if $\kappa(t)=0$,

$-r(t)$ is the remaining service time at time $t$ of a customer being served if $\kappa(t) \in \Pi$, or is equal to 0 if $\kappa(t)=0$,

- $\mathbf{g}(t)=\left(g_{i, \alpha}(t):(i, \alpha) \in \mathcal{S}\right) \in \mathcal{R}^{1 \times J_{c}}$ where $g_{i, \alpha}(t)$ is the number of $(i, \alpha)$-customers in the service facility at time $t$ (who are not being served),

- $\mathbf{n}(t)=\left(n_{i, \alpha}(t):(i, \alpha) \in \mathcal{S}\right) \in \mathcal{R}^{1 \times J_{c}}$ where $n_{i, \alpha}(t)$ is the number of $(i, \alpha)$ customers in the waiting room at time $t$,

$-L(t)$ is the other information of the system.

Let $X_{S}^{e}(t)$ denote the two-dimensional value of (group, class) of $\mathbf{c}^{e}$ at time $t$, or $X_{S}^{e}(t)=$ $(0,0)$ if it does not stay in the system at time $t$. We use the term "the system state at time $\tau_{l, \ell}^{e}$ " to denote $\mathbf{Y}\left(\tau_{l, \ell}^{e}\right)$ if $\ell>0$ or denote $\mathbf{Y}\left(\tau_{l, 0}^{e}-\right)$ if $\ell=0$. For simplicity, we use the notation $(j, \beta, \mathbf{Y})_{l, \ell}^{e}$ to denote the status $\left(X_{S}^{e}\left(\tau_{l, \ell}^{e}\right)=(j, \beta), \mathbf{Y}\left(\tau_{l, \ell}^{e}\right)=\mathbf{Y}\right)$ if $\ell>0$, or the status $\left(X_{S}^{e}\left(\tau_{l, 0}^{e}\right)=(j, \beta), \mathbf{Y}\left(\tau_{l, 0}^{e}-\right)=\mathbf{Y}\right)$ if $\ell=0$.

We use the notation $(\mathbf{g}, \mathbf{n}) \in \mathcal{R}^{1 \times 2 J_{c}}$ to denote a generic value of the vector of the numbers of customers at any time epoch $\left(\mathbf{g}=\left(g_{i, \alpha}:(i, \alpha) \in \mathcal{S}\right), \mathbf{n}=\left(n_{i, \alpha}:(i, \alpha) \in\right.\right.$ $\mathcal{S})$ ). Further we often use the notations $\mathbf{1}(r)=\mathbf{1}\{r>0\}$ and $\mathbf{1}_{i, \alpha}(j, \beta)=\mathbf{1}\{(i, \alpha)=$ $(j, \beta)\}$ where $\mathbf{1}\{\cdot\} \in \mathcal{R}$ is an indicator function and $r$ denotes a generic value of the remaining service time of a customer being served currently.

Note. We assume that at any polling instant $\tau_{l, \ell}^{e}(\ell>0)$, all gates are closed and all customers are in the waiting room, and then immediately after the instant, the gate of the station visited by the server is opened and its customers are admitted into the service facility.

\subsection{The performance measures}

We define two types of the system performance measures of customer $\mathbf{c}^{e}(e=1,2, \ldots)$. First type of them are related to the waiting times of customer $\mathbf{c}^{e}$ in the waiting room. 
We define

$$
C_{W i, \alpha}^{e}(t)= \begin{cases}1, & \text { if } \mathbf{c}^{e} \text { stays in the waiting room as an }(i, \alpha) \text {-customer at time } t \\ 0, & \text { otherwise }\end{cases}
$$

for any $t \geq 0$ and $(i, \alpha) \in \mathcal{S}$. Then we define

$$
\begin{aligned}
H_{i, \alpha}^{e}(k) & =\int_{0}^{\infty} C_{W i, \alpha}^{e}(t) \mathbf{1}\{\kappa(t)=k\} d t, \quad(i, \alpha) \in \mathcal{S}, \quad k \in \Pi, \\
W_{i, \alpha}^{e} & =\sum_{k=1}^{J} H_{i, \alpha}^{e}(k)=\int_{0}^{\infty} C_{W i, \alpha}^{e}(t) d t, \quad(i, \alpha) \in \mathcal{S},
\end{aligned}
$$

where $\mathbf{1}\{\cdot\}$ is an indicator function. $H_{i, \alpha}^{e}(k)$ is the waiting time that $\mathbf{c}^{e}$ spends in the waiting room as an $(i, \alpha)$-customer while the system is in period $k . W_{i, \alpha}^{e}$ is the waiting time that $\mathbf{c}^{e}$ spends in the waiting room as an $(i, \alpha)$-customer.

We would like to obtain the following expected waiting times conditioned on the state of the system at time $\tau_{l, 0}^{e}$.

$$
H_{i, \alpha}(j, \beta, \mathbf{Y}, e, l, k)=E\left[\int_{\tau_{l, 0}^{e}}^{\infty} C_{W i, \alpha}^{e}(t) \mathbf{1}\{\kappa(t)=k\} d t \mid(j, \beta, \mathbf{Y})_{l, 0}^{e}\right]
$$

for $(i, \alpha),(j, \beta) \in \mathcal{S} ; \mathbf{Y} \in \mathcal{E} ; l=0,1,2, \ldots ; k \in \Pi$. (For convenience, we define $H_{i, \alpha}(0,0, \mathbf{Y}, e, l, k)=0$.) Further we define the following related conditional expected waiting times.

$$
\begin{aligned}
& H_{j, \beta}^{1}(\mathbf{Y}, e, l, \ell, k)=E\left[\begin{array}{l|l}
\int_{\tau_{l, \ell}^{e}}^{\tau_{l+1,0}^{e}} C_{W j, \beta}^{e}(t) \mathbf{1}\{\kappa(t)=k\} d t & \begin{array}{c}
(j, \beta, \mathbf{Y})_{l, \ell}^{e} \\
\tau_{l, \ell}^{e}<\tau_{l+1,0}^{e}
\end{array}
\end{array}\right],
\end{aligned}
$$

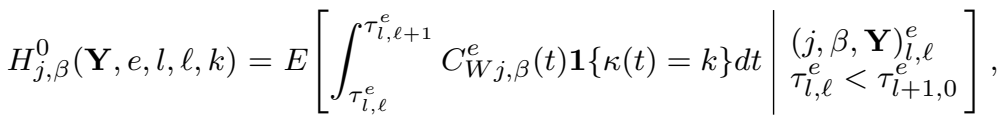

for $(j, \beta) \in \mathcal{S} ; \mathbf{Y} \in \mathcal{E} ; l, \ell=0,1,2, \ldots ; k \in \Pi$. The condition $\tau_{l, \ell}^{e}<\tau_{l+1,0}^{e}$ denotes that during $\left[\tau_{l, 0}^{e}, \tau_{l, \ell}^{e}\right], \mathbf{c}^{e}$ has not been served and its group and class have not been changed. $H_{j, \beta}^{1}(\mathbf{Y}, e, l, \ell, k)$ is the expected waiting time $\mathbf{c}^{e}$ spends during $\left[\tau_{l, \ell}^{e}, \tau_{l+1,0}^{e}\right)$ in a service stage and $H_{j, \beta}^{0}(\mathbf{Y}, e, l, \ell, k)$ is the expected waiting time $\mathbf{c}^{e}$ spends during a service period $\left[\tau_{l, \ell}^{e}, \tau_{l, \ell+1}^{e}\right.$ ) (while the system is in period $k$ ). Then the following equations hold.

\section{Feedback equation.}

$$
\begin{aligned}
& H_{i, \alpha}(j, \beta, \mathbf{Y}, e, l, k) \\
& =\left\{\begin{array}{c}
H_{j, \beta}^{1}(\mathbf{Y}, e, l, 0, k) \\
\quad+E\left[H_{i, \alpha}\left(X_{S}^{e}\left(\tau_{l+1,0}^{e}\right), \mathbf{Y}\left(\tau_{l+1,0}^{e}-\right), e, l+1, k\right) \mid(j, \beta, \mathbf{Y})_{l, 0}^{e}\right], \\
E\left[H_{i, \alpha}\left(X_{S}^{e}\left(\tau_{l+1,0}^{e}\right), \mathbf{Y}\left(\tau_{l+1,0}^{e}-\right), e, l+1, k\right) \mid(j, \beta, \mathbf{Y})_{l, 0}^{e}\right], \quad(i, \alpha) \neq(j, \beta),
\end{array}\right.
\end{aligned}
$$

for $\mathbf{Y}=\left(\kappa_{0}, a_{0}, r, \mathbf{g}, \mathbf{n}, L\right) \in \mathcal{E} ; \quad(i, \alpha),(j, \beta) \in \mathcal{S} ; \quad l=0,1,2, \ldots$ and $k \in \Pi$. 


\section{Polling equation.}

$$
\begin{aligned}
& H_{j, \beta}^{1}(\mathbf{Y}, e, l, \ell, k) \\
& =\left\{\begin{array}{r}
H_{j, \beta}^{0}(\mathbf{Y}, e, l, \ell, k) \\
+E\left[H_{j, \beta}^{1}\left(\mathbf{Y}\left(\tau_{l, \ell+1}^{e}\right), e, l, \ell+1, k\right) \mid(j, \beta, \mathbf{Y})_{l, \ell}^{e}, \tau_{l, \ell}^{e}<\tau_{l+1,0}^{e}\right] \\
\text { if }\left(\kappa_{0} \neq j, \kappa_{0} \in \Pi\right) \text { or }\left(\kappa_{0}=j \in \mathcal{H}_{g}, \ell=0\right), \\
0, \quad \text { if }\left(\kappa_{0}=j \in \mathcal{H}_{e}\right) \text { or }\left(\kappa_{0}=j \in \mathcal{H}_{g}, \ell>0\right) \text { or }\left(\kappa_{0}=0, l=0, \ell=0\right),
\end{array}\right.
\end{aligned}
$$

for $\mathbf{Y}=\left(\kappa_{0}, a_{0}, r, \mathbf{g}, \mathbf{n}, L\right) \in \mathcal{E} ; \quad(j, \beta) \in \mathcal{S} ; \quad l, \ell=0,1,2, \ldots$ and $k \in \Pi$.

The second type of the performance measures are related to the sojourn times of customer $\mathbf{c}^{e}$ in the service facility. We define for any $t \geq 0$ and $(i, \alpha) \in \mathcal{S}$,

$$
C_{F i, \alpha}^{e}(t)=\left\{\begin{aligned}
1, & \text { if } \mathbf{c}^{e} \text { stays in the service facility or receives } \\
\text { a service as an }(i, \alpha) \text {-customer at time } t, & \text { otherwise. }
\end{aligned}\right.
$$

The sojourn time (i.e., the waiting time plus the service time) that $\mathbf{c}^{e}$ spends in the service facility as an $(i, \alpha)$-customer is defined by

$$
F_{i, \alpha}^{e}=\int_{0}^{\infty} C_{F i, \alpha}^{e}(t) d t, \quad(i, \alpha) \in \mathcal{S} .
$$

The expected sojourn time in the service facility conditioned on the state of the system at time $\tau_{l, 0}^{e}$ is defined by

$$
\begin{aligned}
F_{i, \alpha}(j, \beta, \mathbf{Y}, e, l) & =E\left[\int_{\tau_{l, 0}^{e}}^{\infty} C_{F i, \alpha}^{e}(t) d t \mid(j, \beta, \mathbf{Y})_{l, 0}^{e}\right], \\
F_{j, \beta}^{1}(\mathbf{Y}, e, l) & =E\left[\int_{\tau_{l, 0}^{e}}^{\tau_{l+1,0}^{e}} C_{F j, \beta}^{e}(t) d t \mid(j, \beta, \mathbf{Y})_{l, 0}^{e}\right],
\end{aligned}
$$

for $\mathbf{Y} \in \mathcal{E},(i, \alpha),(j, \beta) \in \mathcal{S}$. (For convenience, we define $F_{i, \alpha}(0,0, \mathbf{Y}, e, l)=0$.) Then the feedback equation for $F_{i, \alpha}(\cdot)$ similar to equation (8) holds. (For this type of the performance measures, we do not explicitly set up a polling equation.)

\section{Analysis of Service Periods of Stations}

In this section we obtain the conditional expected waiting times $H_{j, \beta}^{0}(\cdot, e, l, \ell, \cdot)$ of the customer $\mathbf{c}^{e}$ assuming that it is a $(j, \beta)$-customer at epoch $\tau_{l, \ell}^{e}(e=1,2, \ldots ;(j, \beta) \in$ $\mathcal{S} ; l, \ell=0,1,2, \ldots)$. We also consider the expected numbers of customers at the next polling instant $\tau_{l, \ell+1}^{e}$.

Now let us consider an $(i, \alpha)$-customer staying at station $i((i, \alpha) \in \mathcal{S})$. Let $T_{i, \alpha}^{\delta}$ be the total amount of service times the customer receives until the first time it departs from the set of classes $(i, 1), \ldots,(i, \delta)$ at station $i$ after at least receiving its initial service time as an $(i, \alpha)$-customer $\left(\delta=0,1, \ldots, L_{i}\right)$. Let $\bar{T}_{i, \alpha}^{\delta}$ be its expected value and 
$\bar{T}_{i, \alpha}^{\delta}(r)$ be its expected value conditioned on its initial remaining service time $r$ as an $(i, \alpha)$-customer. They satisfy the following equations.

$$
\begin{aligned}
& \bar{T}_{i, \alpha}^{\delta}=E\left[S_{i, \alpha}\right]+\sum_{\beta=1}^{\delta} p_{i, \alpha, i, \beta} \bar{T}_{i, \beta}^{\delta} ; \\
& \bar{T}_{i, \alpha}^{\delta}(r)=r+\sum_{\beta=1}^{\delta} p_{i, \alpha, i, \beta} \bar{T}_{i, \beta}^{\delta} ;
\end{aligned}
$$

for $(i, \alpha) \in \mathcal{S}$ and $\delta=0,1, \ldots, L_{i}$. (The empty sum which arises when $\delta=0$ is equal to 0.) Then let $\varrho_{i, \delta}^{+}=\sum_{\alpha=1}^{\delta} \lambda_{i, \alpha} \bar{T}_{i, \alpha}^{\delta}$.

Let $N_{i, \alpha, k, \gamma}$ be the number of $(k, \gamma)$-customers who arrive during a service period of station $i$ starting with an $(i, \alpha)$-customer, and who still stay at station $k$ at the service period completion epoch. Then its expected value and its expected value conditioned on the remaining service time $r$ of the initial $(i, \alpha)$-customer respectively are denoted by $\bar{N}_{i, \alpha, k, \gamma}$ and $\bar{N}_{i, \alpha, k, \gamma}(r)$. They satisfy the following equations.

$$
\begin{gathered}
\bar{N}_{i, \alpha, k, \gamma}=\left\{\begin{array}{lr}
\lambda_{k, \gamma} E\left[S_{i, \alpha}\right]+p_{i, \alpha, k, \gamma}, & i \in \mathcal{H}_{g}, \\
\lambda_{k, \gamma} E\left[S_{i, \alpha}\right]+p_{i, \alpha, k, \gamma} & \\
\quad+\sum_{\beta=1}^{L_{i}}\left(\lambda_{i, \beta} E\left[S_{i, \alpha}\right]+p_{i, \alpha, i, \beta}\right) \bar{N}_{i, \beta, k, \gamma}, & k \neq i \in \mathcal{H}_{e}, \\
0, & k=i \in \mathcal{H}_{e},
\end{array}\right. \\
\bar{N}_{i, \alpha, k, \gamma}(r)=\left\{\begin{array}{cc}
\lambda_{k, \gamma} r+p_{i, \alpha, k, \gamma}, & i \in \mathcal{H}_{g}, \\
\lambda_{k, \gamma} r+p_{i, \alpha, k, \gamma} & \\
\quad+\sum_{\beta=1}^{L_{i}}\left(\lambda_{i, \beta} r+p_{i, \alpha, i, \beta}\right) \bar{N}_{i, \beta, k, \gamma}, & k \neq i \in \mathcal{H}_{e}, \\
0, & k=i \in \mathcal{H}_{e} .
\end{array}\right.
\end{gathered}
$$

Finally we define the notion related to busy periods, which will be used in Section 4. For $j \in \mathcal{H}_{e P}$ and $\delta=0,1, \ldots, L_{j}$, let a " $(j, \delta)$-busy period" denote a period during a service period of station $j$ completed at the first time when all customers belonging to classes $(j, 1), \ldots,(j, \delta)$ clear. We assume that a $(j, \alpha)$-customer can initiate a $(j, \delta)$-busy period even if $\alpha>\delta$. (Of course, all customers belonging to $(j, \delta+1), \ldots,\left(j, L_{j}\right)$ other than the initial customer do not receive any service during the $(j, \delta)$-busy period.) In particular, a $(j, 0)$-busy period is completed at the first time when a group $j$ customer being served currently completes its service, and a service period of station $j$ is a $\left(j, L_{j}\right)$-busy period. Then the expected length of a $(j, \delta)$-busy period initiated with a $(j, \alpha)$-customer is given by $\bar{T}_{j, \alpha}^{\delta} /\left(1-\varrho_{j, \delta}^{+}\right),\left(\alpha=1, \ldots, L_{j}\right.$ and $\left.\delta=0,1, \ldots, L_{j}\right)$.

3.1 Expressions for $H_{j, \beta}^{0}(\cdot)$

Let $l, \ell=0,1,2, \ldots$ and let $\mathbf{Y}=\left(\kappa_{0}, a_{0}, r, \mathbf{g}, \mathbf{n}, L\right) \in \mathcal{E}$ be the system state at time $\tau_{l, \ell}^{e}$ where $\mathbf{g}=\left(g_{i, \alpha}:(i, \alpha) \in \mathcal{S}\right)$ and $\mathbf{n}=\left(n_{i, \alpha}:(i, \alpha) \in \mathcal{S}\right)$. We assume that $\mathbf{c}^{e}$ is a $(j, \beta)$-customer at this time, i.e., $X_{S}^{e}\left(\tau_{l, \ell}^{e}\right)=(j, \beta)$. For $\kappa_{0} \in \mathcal{H}_{g}$, since we need the value only for $\left(\kappa_{0} \neq j, \ell>0\right)$ or $(\ell=0)$, the other case is not considered.

$$
H_{j, \beta}^{0}(\mathbf{Y}, e, l, \ell, k)= \begin{cases}\sum_{\alpha=1}^{L_{\kappa_{0}}} n_{\kappa_{0}, \alpha} E\left[S_{\kappa_{0}, \alpha}\right], & k=\kappa_{0}, \kappa_{0} \neq j, \ell>0, \\ r+\sum_{\alpha=1}^{L_{\kappa_{0}}} g_{\kappa_{0}, \alpha} E\left[S_{\kappa_{0}, \alpha}\right], & k=\kappa_{0}, \ell=0, \\ 0, & \text { otherwise. }\end{cases}
$$


For $\kappa_{0} \in \mathcal{H}_{e}$, since we need the value only for $\left(\kappa_{0} \neq j\right)$, the other case is not considered.

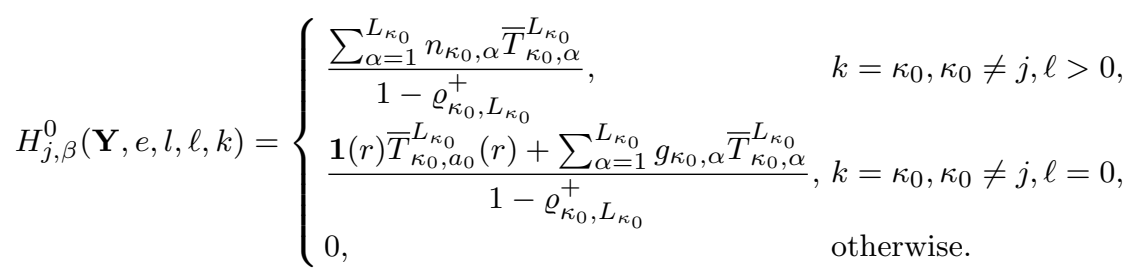

3.2 System State at the Next Polling Instant $\tau_{l, \ell+1}^{e}$

Let $l, \ell=0,1,2, \ldots$ and let $\mathbf{Y}=\left(\kappa_{0}, a_{0}, r, \mathbf{g}, \mathbf{n}, L\right) \in \mathcal{E}$ be the system state at time $\tau_{l, \ell}^{e}$ where $\mathbf{g}=\left(g_{i, \alpha}:(i, \alpha) \in \mathcal{S}\right)$ and $\mathbf{n}=\left(n_{i, \alpha}:(i, \alpha) \in \mathcal{S}\right)$. We consider the system state at the next polling instant $\tau_{l, \ell+1}^{e}$.

When we consider the system state (especially, the numbers of customers) at the next polling instant, we consider the following cases according to $\kappa_{0}$. For $\kappa_{0} \in \mathcal{H}_{g}$, we can show that

$$
\begin{gathered}
E\left[n_{m, \gamma}\left(\tau_{l, \ell+1}^{e}\right) \mid \kappa\left(\tau_{l, \ell+1}^{e}\right)=\kappa_{1},(j, \beta, \mathbf{Y})_{l, \ell}^{e}, \tau_{l, \ell}^{e}<\tau_{l+1,0}^{e}\right] \\
= \begin{cases}n_{m, \gamma}+\sum_{\alpha=1}^{L_{\kappa_{0}}} n_{\kappa_{0}, \alpha} \bar{N}_{\kappa_{0}, \alpha, m, \gamma}, & m \neq \kappa_{0}, \quad(\ell>0), \\
\sum_{\alpha=1}^{L_{\kappa_{0}}} n_{\kappa_{0}, \alpha} \bar{N}_{\kappa_{0}, \alpha, m, \gamma}, & m=\kappa_{0}, \quad(\ell>0), \\
n_{m, \gamma}+\mathbf{1}_{m, \gamma}(j, \beta)+\mathbf{1}(r) \bar{N}_{\kappa_{0}, a_{0}, m, \gamma}(r) & (\ell=0),\end{cases}
\end{gathered}
$$

for any $(j, \beta),(m, \gamma) \in \mathcal{S} ; \kappa_{1} \in \Pi$. For $\kappa_{0} \in \mathcal{H}_{e}$, we have

$$
\begin{aligned}
& E\left[n_{m, \gamma}\left(\tau_{l, \ell+1}^{e}\right) \mid \kappa\left(\tau_{l, \ell+1}^{e}\right)=\kappa_{1},(j, \beta, \mathbf{Y})_{l, \ell}^{e}, \tau_{l, \ell}^{e}<\tau_{l+1,0}^{e}\right]
\end{aligned}
$$

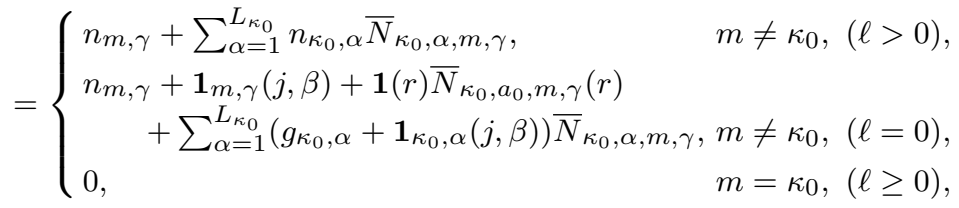

for any $(j, \beta),(m, \gamma) \in \mathcal{S} ; \kappa_{1} \in \Pi$. Further for any $(j, \beta),(m, \gamma) \in \mathcal{S} ; \kappa_{1} \in \Pi$, we have

$$
E\left[g_{m, \gamma}\left(\tau_{l, \ell+1}^{e}\right) \mid \kappa\left(\tau_{l, \ell+1}^{e}\right)=\kappa_{1},(j, \beta, \mathbf{Y})_{l, \ell}^{e}, \tau_{l, \ell}^{e}<\tau_{l+1,0}^{e}\right]=0 .
$$

3.3 The Linear Functional Expressions for the Quantities

From the analysis in this section, we have the following linear functional expressions for the above expectations. In order to obtain these expressions, we define the following constants.

$$
\begin{aligned}
& \mathbf{h}_{10}^{0}(\cdot) \in \mathcal{R}^{2 J_{c} \times 1} ; \varphi^{0}(\cdot) \in \mathcal{R}^{2 \times 1} ; \mathbf{h}_{00}^{0}(\cdot) \in \mathcal{R}^{2 J_{c} \times 1} ; \\
& \mathbf{U}_{1}(\cdot) \in \mathcal{R}^{2 J_{c} \times 2 J_{c}} ; \boldsymbol{v}_{0}(\cdot) \in \mathcal{R}^{2 \times 2 J_{c}} ; \mathbf{U}_{0}(\cdot) \in \mathcal{R}^{2 J_{c} \times 2 J_{c}} ; \mathbf{u}_{0}(\cdot) \in \mathcal{R}^{1 \times 2 J_{c}} .
\end{aligned}
$$

Their detailed definitions are given in Sub-section 9.1. 
Proposition 1 Let $\mathbf{Y}=\left(\kappa_{0}, a_{0}, r, \mathbf{g}, \mathbf{n}, L\right) \in \mathcal{E} ;(j, \beta) \in \mathcal{S} ; \quad e=1,2, \ldots ; l, \ell=$ $0,1,2, \ldots$ and $k, \kappa_{1} \in \Pi$. Then we have

$$
\begin{aligned}
& H_{j, \beta}^{0}(\mathbf{Y}, e, l, \ell, k)= \begin{cases}(\mathbf{g}, \mathbf{n}) \mathbf{h}_{10}^{0}\left(\kappa_{0}, j, k\right), & \ell>0, \\
(r, \mathbf{1}(r)) \boldsymbol{\varphi}^{0}\left(\kappa_{0}, a_{0}, j, k\right)+(\mathbf{g}, \mathbf{n}) \mathbf{h}_{00}^{0}\left(\kappa_{0}, j, k\right), \ell=0,\end{cases} \\
& E\left[\left(\mathbf{g}\left(\tau_{l, \ell+1}^{e}\right), \mathbf{n}\left(\tau_{l, \ell+1}^{e}\right)\right) \mid \kappa\left(\tau_{l, \ell+1}^{e}\right)=\kappa_{1},(j, \beta, \mathbf{Y})_{l, \ell}^{e}, \tau_{l, \ell}^{e}<\tau_{l+1,0}^{e}\right] \\
& =\left\{\begin{array}{lr}
(\mathbf{g}, \mathbf{n}) \mathbf{U}_{1}\left(\kappa_{0}\right), & \ell>0, \\
(r, \mathbf{1}(r)) \boldsymbol{v}_{0}\left(\kappa_{0}, a_{0}\right)+(\mathbf{g}, \mathbf{n}) \mathbf{U}_{0}\left(\kappa_{0}\right)+\mathbf{u}_{0}\left(\kappa_{0}, j, \beta\right), & \ell=0 .
\end{array}\right.
\end{aligned}
$$

\section{Analysis of Service Stages of a Customer}

In this section we obtain the quantities related to each service stage of customer $\mathbf{c}^{e}$. First we obtain the numbers of customers at the first polling instant of the station $\mathbf{c}^{e}$ stays in. Then we obtain the expressions for $H_{j, \beta}^{1}(\cdot, k)$ and $F_{j, \beta}^{1}(\cdot)$ by solving the polling equations. It can be shown that they have the linear functional forms. Finally we obtain the expected values of the system state at the completion epoch of the service stage.

4.1 Numbers of Customers at the First Polling Instant

For any $l=0,1,2, \ldots$ and $j \in \Pi$, let us consider the event that customer $\mathbf{c}^{e}$ arrives at station $j$ at time $\tau_{l, 0}^{e}$. Then let $\tau_{l, M_{l}^{e}}^{e}$ be the first time after $\tau_{l, 0}^{e}$ just when $\mathbf{c}^{e}$ is admitted into the service facility. Basically $\tau_{l, M_{l}^{e}}^{e}$ is the first polling instant of station $j$ after the arrival epoch, except for the following case.

- If $\kappa\left(\tau_{l, 0}^{e}-\right)=j \in \mathcal{H}_{e}$, then $\tau_{l, M_{l}^{e}}^{e}=\tau_{l, 0}^{e}$ and $M_{l}^{e}=0$.

(If $\kappa\left(\tau_{0,0^{-}}^{e}\right)=0$, that is, the system is empty just before $\tau_{0,0}^{e}$, then $\tau_{0, M_{0}^{e}}^{e}$ is equal to the first polling instant $\tau_{0,1}^{e}\left(=\tau_{0,0}^{e}\right)$ and $M_{0}^{e}=1$.) Then we define

$$
\begin{aligned}
& \bar{\nu}_{k, \gamma}^{j, \beta}(\mathbf{Y}, e, l, \ell)=E\left[n_{k, \gamma}\left(\tau_{l, M_{l}^{e}}^{e}\right) \mid(j, \beta, \mathbf{Y})_{l, \ell}^{e}, \tau_{l, \ell}^{e}<\tau_{l+1,0}^{e}\right] \\
& \bar{\nu}^{j, \beta}(\mathbf{Y}, e, l, \ell)=\left(\bar{\nu}_{k, \gamma}^{j, \beta}(\mathbf{Y}, e, l, \ell):(k, \gamma) \in \mathcal{S}\right) \in \mathcal{R}^{1 \times J_{c}},
\end{aligned}
$$

for $(k, \gamma),(j, \beta) \in \mathcal{S} ; \mathbf{Y} \in \mathcal{E}$ and $l, \ell \geq 0$.

In order to obtain the expressions for the above expectation, we define the following constants.

$$
\mathbf{B}_{1}^{j}(\cdot) \in \mathcal{R}^{2 J_{c} \times J_{c}} ; \mathbf{c}^{j}(\cdot) \in \mathcal{R}^{2 \times J_{c}} ; \mathbf{B}_{0}^{j}(\cdot) \in \mathcal{R}^{2 J_{c} \times J_{c}} ; \mathbf{b}_{0}^{j, \beta}(\cdot) \in \mathcal{R}^{1 \times J_{c}} .
$$

Their detailed definitions are given in Sub-section 9.2 .

Proposition 2 The conditional expected numbers of customers at the first polling instant (or arrival epoch) have the following expression.

$$
\overline{\boldsymbol{\nu}}^{j, \beta}(\mathbf{Y}, e, l, \ell)= \begin{cases}(\mathbf{g}, \mathbf{n}) \mathbf{B}_{1}^{j}\left(\kappa_{0}\right), & \ell>0 \\ (r, \mathbf{1}(r)) \mathbf{c}^{j}\left(\kappa_{0}, a_{0}\right)+(\mathbf{g}, \mathbf{n}) \mathbf{B}_{0}^{j}\left(\kappa_{0}\right)+\mathbf{b}_{0}^{j, \beta}\left(\kappa_{0}\right), \ell=0\end{cases}
$$

for $\mathbf{Y}=\left(\kappa_{0}, a_{0}, r, \mathbf{g}, \mathbf{n}, L\right) \in \mathcal{E} ;(j, \beta) \in \mathcal{S}$ and $l, \ell \geq 0$. 
Proof: It can be shown that $\bar{\nu}_{k, \gamma}^{j, \beta}(\cdot)$ satisfies the following polling equation.

$$
\bar{\nu}_{k, \gamma}^{j, \beta}(\mathbf{Y}, e, l, \ell)=\left\{\begin{aligned}
E\left[\bar{\nu}_{k, \gamma}^{j, \beta}\left(\mathbf{Y}\left(\tau_{l, \ell+1}^{e}\right), e, l, \ell+1\right) \mid(j, \beta, \mathbf{Y})_{l, \ell}^{e}, \tau_{l, \ell}^{e}<\tau_{l+1,0}^{e}\right], & \\
& \text { if }\left(\kappa_{0} \neq j, \kappa_{0} \in \Pi\right) \text { or }\left(\kappa_{0}=j \in \mathcal{H}_{g}, \ell=0\right), \\
n_{k, \gamma}, & \text { if }\left(\kappa_{0}=j \in \mathcal{H}_{e}\right) \text { or }\left(\kappa_{0}=j \in \mathcal{H}_{g}, \ell>0\right), \\
\mathbf{1}_{k, \gamma}(j, \beta), & \text { if } \kappa_{0}=0, l=0, \ell=0,
\end{aligned}\right.
$$

for $\mathbf{Y}=\left(\kappa_{0}, a_{0}, r, \mathbf{g}, \mathbf{n}, L\right) \in \mathcal{E} ;(k, \gamma),(j, \beta) \in \mathcal{S}$ and $l, \ell \geq 0$.

We can show that $\bar{\nu}_{k, \gamma}^{j, \beta}(\cdot)$ given by (25) satisfies the polling equation by direct substitution and the use of Proposition 1. The uniqueness of the solution of the polling equation is shown in Section 11.

4.2 The Linear Functional Expressions for $H_{j, \beta}^{1}(\cdot)$ and $F_{j, \beta}^{1}(\cdot)$

Now we give the linear functional expression for the performance measures $H_{j, \beta}^{1}(\cdot)$ and $F_{j, \beta}^{1}(\cdot)$ defined by equations (6) and (12). In order to obtain these expressions we define the following constants.

$$
\begin{aligned}
& \mathbf{h}_{10}^{j}(\cdot) \in \mathcal{R}^{2 J_{c} \times 1} ; \\
& \boldsymbol{\varphi}^{j}(\cdot), \boldsymbol{\eta}^{j, \beta}(\cdot) \in \mathcal{R}^{2 \times 1} ; \mathbf{h}_{00}^{j}(\cdot), \mathbf{f}^{j, \beta}(\cdot) \in \mathcal{R}^{2 J_{c} \times 1} ; h_{01}^{j, \beta}(\cdot), f^{j, \beta}(\cdot) \in \mathcal{R} .
\end{aligned}
$$

Their detailed definitions are given in Sub-section 9.3.

Proposition 3 The expressions for the performance measures $H_{j, \beta}^{1}(\cdot)$ and $F_{j, \beta}^{1}(\cdot)$ have the following linear functional forms.

$$
\begin{aligned}
& H_{j, \beta}^{1}(\mathbf{Y}, e, l, \ell, k) \\
& \quad=\left\{\begin{array}{l}
(\mathbf{g}, \mathbf{n}) \mathbf{h}_{10}^{j}\left(\kappa_{0}, k\right), \\
(r, \mathbf{1}(r)) \varphi^{j}\left(\kappa_{0}, a_{0}, k\right)+(\mathbf{g}, \mathbf{n}) \mathbf{h}_{00}^{j}\left(\kappa_{0}, k\right)+h_{01}^{j, \beta}\left(\kappa_{0}, k\right), \ell=0,
\end{array}\right. \\
& F_{j, \beta}^{1}(\mathbf{Y}, e, l)=(r, \mathbf{1}(r)) \boldsymbol{\eta}^{j, \beta}\left(\kappa_{0}, a_{0}\right)+(\mathbf{g}, \mathbf{n}) \mathbf{f}^{j, \beta}\left(\kappa_{0}\right)+f^{j, \beta}\left(\kappa_{0}\right),
\end{aligned}
$$

for any $(j, \beta) \in \mathcal{S} ; \mathbf{Y}=\left(\kappa_{0}, a_{0}, r, \mathbf{g}, \mathbf{n}, L\right) \in \mathcal{E} ; e=1,2, \ldots ; l, \ell=0,1,2, \ldots$ and $k \in \Pi$.

Proof: For $H_{j, \beta}^{1}(\cdot)$, by directly substituting the expression given by (27) and by using Proposition 1, we can show that it satisfies the polling equation (9). The uniqueness of the solution is shown in Section 11.

For $F_{j, \beta}^{1}(\cdot)$, let $\mathbf{Y}=\left(\kappa_{0}, a_{0}, r, \mathbf{g}, \mathbf{n}, L\right) \in \mathcal{E}\left(\mathbf{g}=\left(g_{i, \alpha}:(i, \alpha) \in \mathcal{S}\right), \mathbf{n}=\left(n_{i, \alpha}:\right.\right.$ $(i, \alpha) \in \mathcal{S}))$ be the system state at time $\tau_{l, 0}^{e}$, and we have

$$
F_{j, \beta}^{1}(\mathbf{Y}, e, l)-E\left[S_{j, \beta}\right]
$$




$$
= \begin{cases}\sum_{\alpha=1}^{L_{j}} n_{j, \alpha} E\left[S_{j, \alpha}\right], & j \in \mathcal{H}_{g F}, \\ \sum_{\alpha=1}^{L_{j}} n_{j, \alpha} E\left[S_{j, \alpha}\right], & j \in \mathcal{H}_{e F} \& \kappa_{0} \neq j, \\ r+\sum_{\alpha=1}^{L_{j}} g_{j, \alpha} E\left[S_{j, \alpha}\right], & j \in \mathcal{H}_{e F} \& \kappa_{0}=j, \\ \sum_{\alpha=1}^{\beta-1} \bar{\nu}_{j, \alpha}^{j, \beta}(\mathbf{Y}, e, l, 0) E\left[S_{j, \alpha}\right]+n_{j, \beta} E\left[S_{j, \beta}\right], & j \in \mathcal{H}_{g P}, \\ \frac{\sum_{\alpha=1}^{\beta-1} \bar{\nu}_{j, \alpha}^{j, \beta}(\mathbf{Y}, e, l, 0) \bar{T}_{j, \alpha}^{\beta-1}+n_{j, \beta} \bar{T}_{j, \beta}^{\beta-1}}{1-\varrho_{j, \beta-1}^{+},}, & j \in \mathcal{H}_{e P} \& \kappa_{0} \neq j, \\ \frac{\mathbf{1}(r) \bar{T}_{\kappa_{0}, a_{0}}^{\beta-1}(r)+\sum_{\alpha=1}^{\beta} g_{j, \alpha} \bar{T}_{j, \alpha}^{\beta-1}}{1-\varrho_{j, \beta-1}^{+}}, & j \in \mathcal{H}_{e P} \& \kappa_{0}=j,\end{cases}
$$

for $\kappa_{0} \in \Pi$, and

$$
F_{j, \beta}^{1}(\mathbf{Y}, e, l)-E\left[S_{j, \beta}\right]=0
$$

for $\kappa_{0}=0(l=0)$.

Note that the quantity $\bar{T}_{j, \alpha}^{\beta-1} /\left(1-\varrho_{j, \beta-1}^{+}\right)$in the above expressions is the expected length of the $(j, \beta-1)$-busy period starting with a $(j, \alpha)$-customer. By substituting the expression (25) into the above expression, the expression (28) is obtained.

4.3 Numbers of customers at the next feedback epoch

Let us consider the customer $\mathbf{c}^{e}$ and its arrival epoch $\tau_{l, 0}^{e}(e=1,2, \ldots ; l=0,1,2, \ldots)$. Now we obtain the conditional expected numbers of customers at its next feedback (or departure) epoch $\tau_{l+1,0}^{e}$ given that $\mathbf{Y}\left(\tau_{l, 0}^{e}-\right)=\mathbf{Y}=\left(\kappa_{0}, a_{0}, r, \mathbf{g}, \mathbf{n}, L\right) \in \mathcal{E}$ and $X_{S}^{e}\left(\tau_{l, 0}^{e}\right)=(j, \beta) \in \mathcal{S}\left(\mathbf{n}=\left(n_{k, \gamma}:(k, \gamma) \in \mathcal{S}\right)\right.$ and $\left.\mathbf{g}=\left(g_{k, \gamma}:(k, \gamma) \in \mathcal{S}\right)\right)$. These quantities are necessary for solving the feedback equations. Recall that the time $\tau_{l, M_{l}^{e}}^{e}\left(\geq \tau_{l, 0}^{e}\right)$ is the first polling instant (or arrival epoch) when the server visits (or already stays at) station $j$ for $\mathbf{c}^{e}$ s service.

\subsubsection{Gated groups}

We consider the case: $j \in \mathcal{H}_{g}$. The number $n_{k, \gamma}\left(\tau_{l+1,0^{-}}^{e}\right)$ is a sum of the following $(k, \gamma)$-customers:

(1) $(k, \gamma)$-customers staying in the system at $\tau_{l, M_{l}^{e}}^{e}($ if $k \neq j)$, and

(2) $(k, \gamma)$-customers arriving from outside or by feedback while $\mathbf{c}^{e}$ stays in the service facility.

Then we have

$$
\begin{aligned}
E & {\left[n_{k, \gamma}\left(\tau_{l+1,0}^{e}-\right) \mid(j, \beta, \mathbf{Y})_{l, 0}^{e}\right] } \\
= & \begin{cases}\bar{\nu}_{k, \gamma}^{j, \beta}(\mathbf{Y}, e, l, 0)+\lambda_{k, \gamma} F_{j, \beta}^{1}(\mathbf{Y}, e, l)+\sum_{\alpha=1}^{L_{j}} n_{j, \alpha} p_{j, \alpha, k, \gamma}, k \neq j, j \in \mathcal{H}_{g F}, \\
\bar{\nu}_{k, \gamma}^{j, \beta}(\mathbf{Y}, e, l, 0)+\lambda_{k, \gamma} F_{j, \beta}^{1}(\mathbf{Y}, e, l) \\
\quad+\sum_{\alpha=1}^{\beta-1} \bar{\nu}_{j, \alpha}^{j, \beta}(\mathbf{Y}, e, l, 0) p_{j, \alpha, k, \gamma}+n_{j, \beta} p_{j, \beta, k, \gamma}, & k \neq j, j \in \mathcal{H}_{g P}, \\
\lambda_{j, \gamma} F_{j, \beta}^{1}(\mathbf{Y}, e, l)+\sum_{\alpha=1}^{L_{j}} n_{j, \alpha} p_{j, \alpha, j, \gamma}, & k=j, j \in \mathcal{H}_{g F}, \\
\lambda_{j, \gamma} F_{j, \beta}^{1}(\mathbf{Y}, e, l) & k=j, j \in \mathcal{H}_{g P} .\end{cases}
\end{aligned}
$$


The number $g_{k, \gamma}\left(\tau_{l+1,0^{-}}^{e}\right)$ is equal to

(1) 0 (for the case $k \neq j$ ), that is, none of $(k, \gamma)$-customers is in the service facility at $\tau_{l+1,0^{e}}^{e}$; or

(2) the number of $(k, \gamma)$-customers staying in the system at $\tau_{l, M_{l}^{e}}^{e}$ and not served before $\mathbf{c}^{e}$ (for the case $k=j$ ).

Then we have

$$
\begin{aligned}
& E\left[g_{k, \gamma}\left(\tau_{l+1,0}^{e}-\right) \mid(j, \beta, \mathbf{Y})_{l, 0}^{e}\right] \\
& \quad= \begin{cases}0, & k \neq j, \\
\bar{\nu}_{j, \gamma}^{j, \beta}(\mathbf{Y}, e, l, 0)-\left(\mathbf{1}_{j, \gamma}(j, \beta)+n_{j, \gamma}\right), & k=j, j \in \mathcal{H}_{g F}, \\
0, & k=j, \gamma<\beta, j \in \mathcal{H}_{g P}, \\
\bar{\nu}_{j, \beta}^{j, \beta}(\mathbf{Y}, e, l, 0)-\left(1+n_{j, \beta}\right), & k=j, \gamma=\beta, j \in \mathcal{H}_{g P}, \\
\bar{\nu}_{j, \gamma}^{j, \beta}(\mathbf{Y}, e, l, 0), & k=j, \gamma>\beta, j \in \mathcal{H}_{g P} .\end{cases}
\end{aligned}
$$

\subsubsection{Exhaustive FCFS groups}

We consider the case: $j \in \mathcal{H}_{e F}$. In this case, $(k, \gamma)$-customers (for $k \neq j$ ) in the waiting room at $\tau_{l+1,0}^{e}$ are composed of

(1) $(k, \gamma)$-customers staying in the system at $\tau_{l, M_{l}^{e}}^{e}$, and

(2) $(k, \gamma)$-customers arriving from outside or by feedback while $\mathbf{c}^{e}$ stays in the service facility.

None of $j$-customers is in the waiting room at $\tau_{l+1,0}^{e}$. Then we have

$$
\begin{aligned}
& E\left[n_{k, \gamma}\left(\tau_{l+1,0}^{e}-\right) \mid(j, \beta, \mathbf{Y})_{l, 0}^{e}\right] \\
& = \begin{cases}n_{k, \gamma}+\lambda_{k, \gamma} F_{j, \beta}^{1}(\mathbf{Y}, e, l)+\mathbf{1}(r) p_{j, a_{0}, k, \gamma}+\sum_{\alpha=1}^{L_{j}} g_{j, \alpha} p_{j, \alpha, k, \gamma}, & k \neq j,(* 1), \\
\bar{\nu}_{k, \gamma}^{j, \beta}(\mathbf{Y}, e, l, 0)+\lambda_{k, \gamma} F_{j, \beta}^{1}(\mathbf{Y}, e, l)+\sum_{\alpha=1}^{L_{j}} n_{j, \alpha} p_{j, \alpha, k, \gamma}, & k \neq j,(* 2), \\
\lambda_{k, \gamma} E\left[S_{j, \beta}\right], & k \neq j,(* 3), \\
0, & k=j,\end{cases}
\end{aligned}
$$

where $(* 1) \kappa_{0}=j ;(* 2) \kappa_{0} \in \Pi \backslash\{j\} ;(* 3) \kappa_{0}=0$. Then $(j, \gamma)$-customers in the service facility at $\tau_{l+1,0}^{e}$ are composed of

(1) $(j, \gamma)$-customers staying in the system at $\tau_{l, M_{l}^{e}}^{e}$ and not served before $\mathbf{c}^{e}$, and

(2) $(j, \gamma)$-customers arriving from outside or by feedback while $\mathbf{c}^{e}$ stays in the service facility.

Obviously none of $k$-customers $(k \neq j)$ is in the service facility at $\tau_{l+1,0}^{e}$. Then we have

$$
\begin{aligned}
E & {\left[g_{k, \gamma}\left(\tau_{l+1,0}^{e}-\right) \mid(j, \beta, \mathbf{Y})_{l, 0}^{e}\right] } \\
= & \begin{cases}0, & k \neq j, \\
\lambda_{j, \gamma} F_{j, \beta}^{1}(\mathbf{Y}, e, l)+\mathbf{1}(r) p_{j, a_{0}, j, \gamma}+\sum_{\alpha=1}^{L_{j}} g_{j, \alpha} p_{j, \alpha, j, \gamma}, & k=j,(* 1), \\
\bar{\nu}_{j, \gamma}^{j, \beta}(\mathbf{Y}, e, l, 0)-n_{j, \gamma}-\mathbf{1}_{j, \gamma}(j, \beta) & \\
\quad+\lambda_{j, \gamma} F_{j, \beta}^{1}(\mathbf{Y}, e, l)+\sum_{\alpha=1}^{L_{j}} n_{j, \alpha} p_{j, \alpha, j, \gamma}, & k=j,(* 2), \\
\lambda_{j, \gamma} E\left[S_{j, \beta}\right], & k=j,(* 3),\end{cases}
\end{aligned}
$$

where $(* 1) \kappa_{0}=j ;(* 2) \kappa_{0} \in \Pi \backslash\{j\} ;(* 3) \kappa_{0}=0$. 


\subsubsection{Exhaustive priority groups}

We consider the case: $j \in \mathcal{H}_{e P}$. Let $N_{j, \alpha, k, \gamma}^{(j, \delta)}$ be the number of $(k, \gamma)$-customers who arrive (from outside or by feedback) during a $(j, \delta)$-busy period starting with a $(j, \alpha)$ customer, and who still stay at station $k$ at the end of the period $((k \neq j)$ or $(k=j$ and $\gamma>\delta)$ ). Then let $\bar{N}_{j, \alpha, k, \gamma}^{(j, \delta)}$ be its expected value and let $\bar{N}_{j, \alpha, k, \gamma}^{(j, \delta)}(r)$ be its expected value conditioned on the remaining service time $r$ of the initial $(j, \alpha)$-customer. Then

$$
\begin{aligned}
& \bar{N}_{j, \alpha, k, \gamma}^{(j, \delta)}=\lambda_{k, \gamma} E\left[S_{j, \alpha}\right]+p_{j, \alpha, k, \gamma}+\sum_{\delta^{\prime}=1}^{\delta}\left(\lambda_{j, \delta^{\prime}} E\left[S_{j, \alpha}\right]+p_{j, \alpha, j, \delta^{\prime}}\right) \bar{N}_{j, \delta^{\prime}, k, \gamma}^{(j, \delta)}, \\
& \bar{N}_{j, \alpha, k, \gamma}^{(j, \delta)}(r)=\lambda_{k, \gamma} r+p_{j, \alpha, k, \gamma}+\sum_{\delta^{\prime}=1}^{\delta}\left(\lambda_{j, \delta^{\prime}} r+p_{j, \alpha, j, \delta^{\prime}}\right) \bar{N}_{j, \delta^{\prime}, k, \gamma}^{(j, \delta)}
\end{aligned}
$$

for $(j, \alpha),(k, \gamma) \in \mathcal{S} ; \delta=0,1, \ldots, L_{j}(\gamma>\delta$ if $k=j)$. Then we have

$$
\begin{aligned}
& E {\left[n_{k, \gamma}\left(\tau_{l+1,0}^{e}-\right) \mid(j, \beta, \mathbf{Y})_{l, 0}^{e}\right] } \\
&= \begin{cases}n_{k, \gamma}+\mathbf{1}(r) \bar{N}_{j, a_{0}, k, \gamma}^{(j, \beta-1)}(r)+\sum_{\alpha=1}^{\beta} g_{j, \alpha} \bar{N}_{j, \alpha, k, \gamma}^{(j, \beta-1)}+\lambda_{k, \gamma} E\left[S_{j, \beta}\right], & k \neq j,(* 1), \\
\bar{\nu}_{k, \gamma}^{j, \beta}(\mathbf{Y}, e, l, 0)+\sum_{\alpha-1}^{\beta-1} \bar{\nu}_{j, \alpha}^{j, \beta}(\mathbf{Y}, e, l, 0) \bar{N}_{j, \alpha, k, \gamma}^{(j, \beta-1)} & k \neq j,(* 2), \\
\quad+n_{j, \beta} \bar{N}_{j, \beta, k, \gamma}^{(j, \beta-1)}+\lambda_{k, \gamma} E\left[S_{j, \beta}\right], & k \neq j,(* 3), \\
\lambda_{k, \gamma} E\left[S_{j, \beta}\right], & k=j, \\
0, & \end{cases}
\end{aligned}
$$

where $(* 1) \kappa_{0}=j ;(* 2) \kappa_{0} \in \Pi \backslash\{j\} ;(* 3) \kappa_{0}=0$. (Note that none of customers arrive by feedback during $\mathbf{c}^{e}$ 's service.) Further the explanation for $(j, \gamma)$-customers in the service facility at the completion epoch of the sojourn time (related to) $F_{j, \beta}^{1}(\mathbf{Y}, e, l$ ) is similar to that for $j \in \mathcal{H}_{e F}$ except that in this case $(j, \gamma)$-customers $(\gamma<\beta)$ are cleared from the system when $\mathbf{c}^{e}$ starts service. Then we have

$$
\begin{aligned}
& E\left[g_{k, \gamma}\left(\tau_{l+1,0}^{e}-\right) \mid(j, \beta, \mathbf{Y})_{l, 0}^{e}\right] \\
& = \begin{cases}0, & k \neq j, \\
\lambda_{j, \gamma} E\left[S_{j, \beta}\right], & k=j,(* 0), \\
\mathbf{1}(r) \bar{N}_{j, a_{0}, j, \beta}^{(j, \beta-1)}(r)+\sum_{\alpha=1}^{\beta} g_{j, \alpha} \bar{N}_{j, \alpha, j, \beta}^{(j, \beta-1)}+\lambda_{j, \beta} E\left[S_{j, \beta}\right], & k=j,(* 1 a), \\
g_{j, \gamma}+\mathbf{1}(r) \bar{N}_{j, a_{0}, j, \gamma}^{(j, \beta-1)}(r)+\sum_{\alpha=1}^{\beta} g_{j, \alpha} \bar{N}_{j, \alpha, j, \gamma}^{(j, \beta-1)}+\lambda_{j, \gamma} E\left[S_{j, \beta}\right], & k=j,(* 1 b), \\
\bar{\nu}_{j, \beta}^{j, \beta}(\mathbf{Y}, e, l, 0)-n_{j, \beta}-1+\sum_{\alpha=1}^{\beta-1} \bar{\nu}_{j, \alpha}^{j, \beta}(\mathbf{Y}, e, l, 0) \bar{N}_{j, \alpha, j, \beta}^{(j, \beta-1)} & \\
\quad+n_{j, \beta} \bar{N}_{j, \beta, j, \beta}^{(j, \beta-1)}+\lambda_{j, \beta} E\left[S_{j, \beta}\right], & k=j,(* 2 a), \\
\bar{\nu}_{j, \gamma}^{j, \beta}(\mathbf{Y}, e, l, 0)+\sum_{\alpha-1}^{\beta-1} \bar{\nu}_{j, \alpha}^{j, \beta}(\mathbf{Y}, e, l, 0) \bar{N}_{j, \alpha, j, \gamma}^{(j, \beta-1)} & k=j,(* 2 b), \\
\quad+n_{j, \beta} \bar{N}_{j, \beta, j, \gamma}^{(j, \beta-1)}+\lambda_{j, \gamma} E\left[S_{j, \beta}\right], & k=j,(* 3), \\
\lambda_{j, \gamma} E\left[S_{j, \beta}\right], & \end{cases}
\end{aligned}
$$

where $(* 0) \gamma<\beta, \kappa_{0} \in \Pi ;(* 1 a) \gamma=\beta, \kappa_{0}=j ;(* 1 b) \gamma>\beta, \kappa_{0}=j ;(* 2 a) \gamma=\beta, \kappa_{0} \in$ $\Pi \backslash\{j\} ;(* 2 b) \gamma>\beta, \kappa_{0} \in \Pi \backslash\{j\} ;(* 3) \kappa_{0}=0$.

\subsubsection{Linear functional expressions for the quantities}

Similar to the quantities given previously, we have the following linear functional expressions for the expected numbers of customers at the next feedback epoch. In order 
to obtain these expressions we define the following constants.

$$
\boldsymbol{v}^{j, \beta}(\cdot) \in \mathcal{R}^{2 \times 2 J_{c}} ; \mathbf{U}^{j, \beta}(\cdot) \in \mathcal{R}^{2 J_{c} \times 2 J_{c}} ; \mathbf{u}^{j, \beta}(\cdot) \in \mathcal{R}^{1 \times 2 J_{c}} .
$$

Their detailed definitions are given in Sub-section 9.4.

Proposition 4 Let $\mathbf{Y}=\left(\kappa_{0}, a_{0}, r, \mathbf{g}, \mathbf{n}, L\right) \in \mathcal{E} ;(j, \beta) \in \mathcal{S} ; \quad e=1,2, \ldots ;$ and $l=$ $0,1,2, \ldots$ Then

$$
\begin{aligned}
& E\left[\left(\mathbf{g}\left(\tau_{l+1,0}^{e}-\right), \mathbf{n}\left(\tau_{l+1,0}^{e}-\right)\right) \mid(j, \beta, \mathbf{Y})_{l, 0}^{e}\right] \\
& \quad=(r, \mathbf{1}(r)) \boldsymbol{v}^{j, \beta}\left(\kappa_{0}, a_{0}\right)+(\mathbf{g}, \mathbf{n}) \mathbf{U}^{j, \beta}\left(\kappa_{0}\right)+\mathbf{u}^{j, \beta}\left(\kappa_{0}\right) .
\end{aligned}
$$

Proof: This expression can be easily obtained by substituting the expressions (25) and (28) into the expressions obtained in this subsection.

\section{The Linear Functional Expressions}

In this section we obtain the expressions for the performance measures $H_{i, \alpha}(\cdot), F_{i, \alpha}(\cdot)$ by solving the feedback equations. As the expressions previously derived, it will be shown that these expressions have the linear functional forms. In order to obtain these expressions we define the following constants.

$$
\boldsymbol{\varphi}_{i, \alpha}(\cdot), \boldsymbol{\eta}_{i, \alpha}(\cdot) \in \mathcal{R}^{2 \times 1} ; \mathbf{w}_{i, \alpha}(\cdot), \mathbf{f}_{i, \alpha}(\cdot) \in \mathcal{R}^{2 J_{c} \times 1} ; w_{i, \alpha}(\cdot), f_{i, \alpha}(\cdot) \in \mathcal{R}
$$

Their detailed definitions are given in Sub-section 9.5.

Proposition 5 The expressions for the performance measures $H_{i, \alpha}(\cdot)$ and $F_{i, \alpha}(\cdot)$ have the following linear functional forms.

$$
\begin{aligned}
H_{i, \alpha}(j, \beta, \mathbf{Y}, e, l, k)=( & r, \mathbf{1}(r)) \boldsymbol{\varphi}_{i, \alpha}\left(j, \beta, \kappa_{0}, a_{0}, k\right) \\
& +(\mathbf{g}, \mathbf{n}) \mathbf{w}_{i, \alpha}\left(j, \beta, \kappa_{0}, k\right)+w_{i, \alpha}\left(j, \beta, \kappa_{0}, k\right), \\
F_{i, \alpha}(j, \beta, \mathbf{Y}, e, l)=(r, & \mathbf{1}(r)) \boldsymbol{\eta}_{i, \alpha}\left(j, \beta, \kappa_{0}, a_{0}\right) \\
& +(\mathbf{g}, \mathbf{n}) \mathbf{f}_{i, \alpha}\left(j, \beta, \kappa_{0}\right)+f_{i, \alpha}\left(j, \beta, \kappa_{0}\right),
\end{aligned}
$$

for $\mathbf{Y}=\left(\kappa_{0}, a_{0}, r, \mathbf{g}, \mathbf{n}, L\right) \in \mathcal{E} ; \quad e=1,2, \ldots ; l=0,1,2, \ldots ;(i, \alpha),(j, \beta) \in \mathcal{S}$ and $k \in \Pi$.

Proof: By directly substituting the expressions given in (38) and (39) and by using Propositions 3 and 4 , we can show that they respectively satisfy the feedback equations given in Section 2. The uniqueness of the solutions is shown by the similar method given in Hirayama (2003), by virtue of the linear functional expressions for the quantities. 


\section{Steady State Values}

Let us consider the system operating under any scheduling algorithm defined in Section 2. In this section, we would like to evaluate the following values for $(i, \alpha),(j, \beta) \in \mathcal{S}$ :

$$
\bar{w}_{i, \alpha}(j, \beta)=\lim _{N \rightarrow \infty} \frac{1}{N} \sum_{e=1}^{N} E\left[W_{i, \alpha}^{e}+F_{i, \alpha}^{e} \mid X_{S}^{e}\left(\tau_{0,0}^{e}\right)=(j, \beta)\right]
$$

$\bar{w}_{i, \alpha}(j, \beta)$ denotes the average sojourn time that any customer arriving from outside the system as a $(j, \beta)$-customer spend as an $(i, \alpha)$-customer during its stay in the system. The time average value of the state of the system is also defined by:

$$
\tilde{\mathbf{Y}}^{k}=\left(k \tilde{q}^{k}, \tilde{a}^{k}, \tilde{r}^{k}, \tilde{\mathbf{g}}^{k}, \tilde{\mathbf{n}}^{k}, \tilde{L}^{k}\right)=\lim _{t \rightarrow \infty} \frac{1}{t} \int_{0}^{t} E[\mathbf{Y}(s) \mathbf{1}\{\kappa(s)=k\}] d s
$$

for $k \in \Pi \cup\{0\}$, where $\tilde{\mathbf{g}}^{k}=\left(\tilde{g}_{i, \alpha}^{k}:(i, \alpha) \in \mathcal{S}\right)$ and $\tilde{\mathbf{n}}^{k}=\left(\tilde{n}_{i, \alpha}^{k}:(i, \alpha) \in \mathcal{S}\right)$. We assume that all of the time averages and the customer averages defined in this section exist.

Then in order to investigate these average values in detail, we further define the average sojourn times and the time average values as follows:

$$
\begin{aligned}
\bar{H}_{i, \alpha}(j, \beta, k) & =\lim _{N \rightarrow \infty} \frac{1}{N} \sum_{e=1}^{N} E\left[H_{i, \alpha}^{e}(k) \mid X_{S}^{e}\left(\tau_{0,0}^{e}\right)=(j, \beta)\right], \\
\bar{F}_{i, \alpha}(j, \beta) & =\lim _{N \rightarrow \infty} \frac{1}{N} \sum_{e=1}^{N} E\left[F_{i, \alpha}^{e} \mid X_{S}^{e}\left(\tau_{0,0}^{e}\right)=(j, \beta)\right], \\
\tilde{q}^{\kappa_{0}, a_{0}} & =\lim _{t \rightarrow \infty} \frac{1}{t} \int_{0}^{t} E\left[\mathbf{1}\left\{(\kappa(s), a(s))=\left(\kappa_{0}, a_{0}\right)\right\}\right] d s, \\
\tilde{r}^{\kappa_{0}, a_{0}} & =\lim _{t \rightarrow \infty} \frac{1}{t} \int_{0}^{t} E\left[r(s) \mathbf{1}\left\{(\kappa(s), a(s))=\left(\kappa_{0}, a_{0}\right)\right\}\right] d s,
\end{aligned}
$$

for $(i, \alpha),(j, \beta) \in \mathcal{S} ; k \in \Pi ;\left(\kappa_{0}, a_{0}\right) \in \mathcal{S} \cup\{(0,0)\}$. Then we have

Proposition 6 The average numbers of customers $\left(\tilde{\mathbf{g}}^{k}, \tilde{\mathbf{n}}^{k}\right)$ and the average sojourn times $\bar{H}_{i, \alpha}(j, \beta, k)$ and $\bar{F}_{i, \alpha}(j, \beta)$ have the following relations. For $(i, \alpha) \in \mathcal{S}$ and $k \in \Pi$,

$$
\begin{aligned}
& \tilde{n}_{i, \alpha}^{k}=\sum_{(j, \beta) \in \mathcal{S}} \lambda_{j, \beta} \bar{H}_{i, \alpha}(j, \beta, k) ; \\
& \tilde{g}_{i, \alpha}^{i}=\sum_{(j, \beta) \in \mathcal{S}} \lambda_{j, \beta} \bar{F}_{i, \alpha}(j, \beta)-\tilde{q}^{i, \alpha} \\
& \tilde{g}_{i, \alpha}^{k}=0, \quad(k \neq i) ; \quad \tilde{n}_{i, \alpha}^{0}=0 ; \quad \tilde{g}_{i, \alpha}^{0}=0 .
\end{aligned}
$$

These expressions simply come from the Little's formula. Then we can get the following proposition that relates the average numbers of customers and the average sojourn times. 
Proposition 7 Let us define the constants $\tilde{\varphi}_{i, \alpha}(j, \beta, k)$ and $\tilde{\eta}_{i, \alpha}(j, \beta),((i, \alpha),(j, \beta) \in$ $\mathcal{S} ; k \in \Pi$ ) whose precise definitions are given in Sub-section 9.6 (Equations (54) and (55)). Then we have

$$
\begin{aligned}
\bar{H}_{i, \alpha}(j, \beta, k) & =\tilde{\varphi}_{i, \alpha}(j, \beta, k)+\sum_{\kappa_{0} \in \Pi}\left(\tilde{\mathbf{g}}^{\kappa_{0}}, \tilde{\mathbf{n}}^{\kappa_{0}}\right) \mathbf{w}_{i, \alpha}\left(j, \beta, \kappa_{0}, k\right), \\
\bar{F}_{i, \alpha}(j, \beta) & =\tilde{\eta}_{i, \alpha}(j, \beta)+\sum_{\kappa_{0} \in \Pi}\left(\tilde{\mathbf{g}}^{\kappa_{0}}, \tilde{\mathbf{n}}^{\kappa_{0}}\right) \mathbf{f}_{i, \alpha}\left(j, \beta, \kappa_{0}\right),
\end{aligned}
$$

for $(i, \alpha),(j, \beta) \in \mathcal{S}$ and $k \in \Pi$.

Proof: These expressions can be obtained by applying the averaging procedures and the PASTA property to the expressions in Proposition 5.

Note. By combining the expressions given in Proposition 6 and the expressions given in Proposition 7 we can obtain the linear equations for the (unknown) numbers of customers $\left(\tilde{\mathbf{g}}^{k}, \tilde{\mathbf{n}}^{k}\right)$. The detailed equations are given in Section 10.

Proposition 8 The average sojourn times are given by

$$
\bar{w}_{i, \alpha}(j, \beta)=\sum_{k \in \Pi} \bar{H}_{i, \alpha}(j, \beta, k)+\bar{F}_{i, \alpha}(j, \beta), \quad((i, \alpha),(j, \beta) \in \mathcal{S}) .
$$

Note. In Section 10 we give an algorithm for calculating the average numbers of customers and the average sojourn times, and its computational complexity.

\section{A Packet scheduling Problem}

In this section we consider a packet scheduling problem in communication networks where five different types of packet-based traffic share a single network resource. Type 1-1 is a mission-critical traffic with the highest importance that constitutes category 1 traffic. Types 2-1 and 2-2 constitute category 2 that carries different types of traffic

Table 1 Statistical quantities for all types of the packets ${ }^{a, b}$

\begin{tabular}{ccccccc}
\hline Category & Type & M.R.R. & V.R.R. & M.Q.S. & V.Q.S. & R.O.R. \\
\hline \hline Category 1 & Type 1-1 & 1.00000 & 0.50000 & 1.00 & 0.50 & 0.21255 \\
\hline Category 2 & Type 2-1 & 3.04005 & 3.67368 & 0.25 & 0.10 & 0.21538 \\
& Type 2-2 & 3.04005 & 3.67368 & 0.50 & 0.20 & 0.21538 \\
\hline \multirow{2}{*}{ Category 3 } & Type 3-1 & 5.03456 & 9.77386 & 0.25 & 0.10 & 0.17835 \\
& Type 3-2 & 5.03456 & 9.77386 & 0.50 & 0.20 & 0.17835 \\
\hline
\end{tabular}

${ }^{a}$ M.R.R. $=$ Mean of Resource Requirement time; V.R.R. = Variance of Resource Requirement time; M.Q.S.= Mean Quantum (Weight) Size; V.Q.S.= Variance of Quantum Size; R.O.R.= (Relative) Resource Occupancy Ratio.

$b$ For simplicity, we first determine the service quanta per stage (M.Q.S.s and V.Q.S.s) and the feedback probabilities for all types, and then calculate the means and the variances of the resource requirement times (M.R.R.s and V.R.R.s). Type 2-1 and type 2-2 have the same resource requirements and assigned the different weights (quanta). The same explanation can be applied to type 3-1 and type 3-2. 


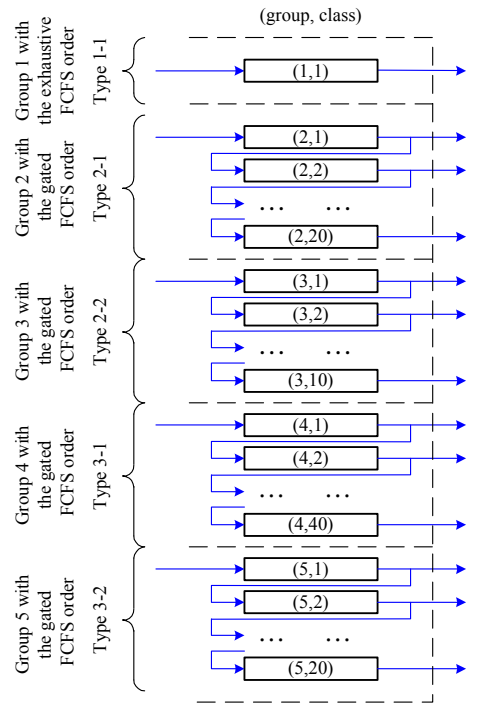

A-1, A-2 and A-4 scheduling algorithms

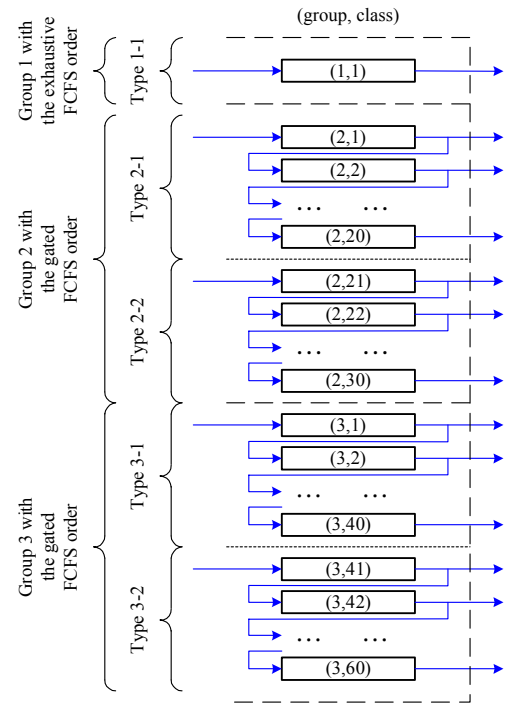

A-3 scheduling algorithm

Fig. 2 Diagrams of the Resource Requirement Paths in the scheduling algorithms

with medium importance. Types 3-1 and 3-2 constitute category 3 that carries different types of normal traffic. Their statistical quantities are listed in Table 1. Their arrival rates are varied in order to obtain the mean response times (i.e. sojourn times) for different values of the resource utilization $\rho$.

We assume that the resource requirement time (i.e. overall service time) of each packet may be divided into multiple quanta each of which can be allocated the resource individually. That is, we consider "positive quanta algorithms." In multimedia networks with various types of traffic, delay sensitive real time traffic or mission critical traffic is frequently necessary to be transmitted before the other normal data traffic. The important traffic can be preferentially treated by allocating the resource more often than the other traffic in the following manners.

- PT1: Allocate the resource more often by arranging the polling probabilities.

- PT2: Allocate the resource by the exhaustive rule.

- PT3: Assign the larger sizes of quanta.

We consider the following four scheduling algorithms (A-1, ., A-4). In the following diagrams, a value above an arrow is a polling probability that the server moves from a type (or a category) at the left of the arrow to a type (or a category) at its right. If no value is above an arrow, it is assumed to be 1 .

A-1. All types are allocated the resource in the following round robin (cyclic) fashion.

$$
\text { type } 1-1 \rightarrow \text { type } 2-1 \rightarrow \text { type } 2-2 \rightarrow \text { type } 3-1 \rightarrow \text { type } 3-2 \rightarrow \text { type } 1-1 \rightarrow \cdots
$$

A-2. Type 1-1 and the other types are alternately allocated the resource as follows.

$$
\text { type } 1-1 \rightarrow\left\{\begin{array}{l}
\stackrel{0.3}{\rightarrow} \text { type } 2-1 \rightarrow \\
\stackrel{0.3}{\longrightarrow} \text { type } 2-2 \rightarrow \\
\underset{0.2}{\longrightarrow} \text { type } 3-1 \rightarrow \\
\stackrel{0.2}{\longrightarrow} \text { type } 3-2 \rightarrow
\end{array}\right\} \rightarrow \text { type } 1-1 \rightarrow \cdots
$$



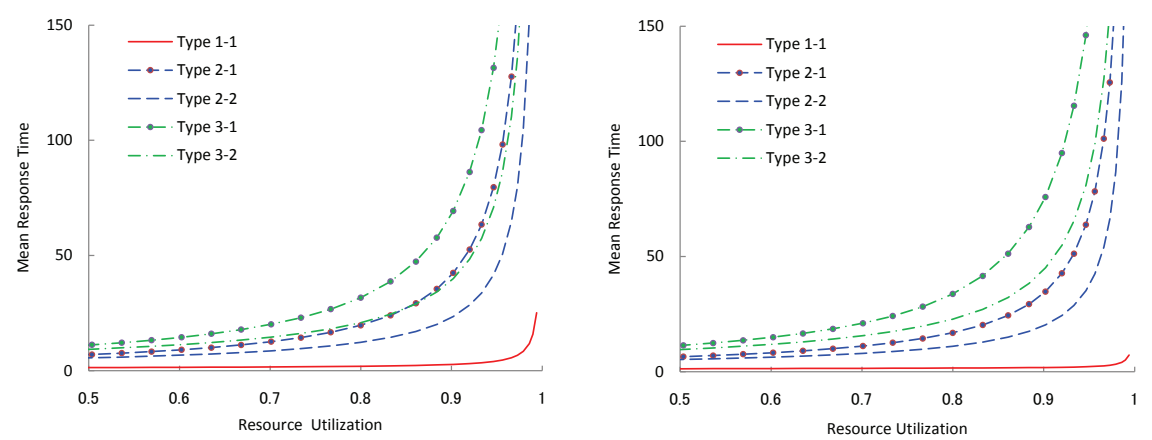

Fig. 3 MRTs for A-1 (left) and MRTs for A-2 (right)

A-3. Category 1 and the other categories are alternately allocated the resource as follows.

$$
\text { category } 1 \rightarrow\left\{\begin{array}{l}
\stackrel{0.6}{\rightarrow} \text { category } 2 \rightarrow \\
\stackrel{0.4}{\rightarrow} \text { category } 3 \rightarrow
\end{array}\right\} \rightarrow \text { category } 1 \rightarrow \cdots
$$

A-4. All types are allocated the resource in the following manner.

$$
\text { type } 1-1 \rightarrow\left\{\begin{array}{l}
\stackrel{0.5}{\longrightarrow} \text { type } 2-1 \rightarrow\left\{\begin{array}{l}
\frac{0.5}{\longrightarrow} \text { type } 3-1 \rightarrow \\
\stackrel{0.5}{\longrightarrow} \longrightarrow \rightarrow
\end{array}\right\} \rightarrow \\
\stackrel{0.5}{\longrightarrow} \text { type } 2-2 \rightarrow\left\{\begin{array}{l}
\frac{0.5}{\longrightarrow} \text { type } 3-2 \rightarrow \\
\stackrel{0.5}{\longrightarrow} \longrightarrow \longrightarrow
\end{array}\right\} \rightarrow
\end{array}\right\} \rightarrow \text { type } 1-1 \rightarrow \cdots
$$

In all of the scheduling algorithms, type 1-1 (category 1) has the exhaustive allocation rule, and all the other types (or categories in A-3) have the gated allocation rule. All quanta of packets in each type (or each category in A-3) are allocated the resource in the FCFS order inside the type (or, respectively, the category). The service order in every category in A-3 is also called the DRR (discriminatory round robin) (Hirayama, 2010).

The resource requirement paths of packets in the scheduling algorithms and their correspondences to the service stages in the queueing model defined in Section 2 are given in Fig.2. Each square box denotes a quantum of each packet that corresponds to a service in a service stage of each customer whose (group, class) is denoted by a pair of numbers in the box. Each arrow denotes a flow of each packet, and it may branch off at an end of a quantum, denoting that each packet receiving the quantum completes its overall resource requirements or requires more quanta probabilistically. For example, type 1-1 receives only one quantum that corresponds to a service in a service stage of class 1 in group 1, while type 3-2 receives at most 20 quanta that correspond to services in service stages of classes $1, \cdots, 20$ in group 5 for A-1, A-2 or A-4; or services in service stages of classes $41, \cdots, 60$ in group 3 for A-3.

In Figs. 3 and 4, the mean response times (MRTs) for all types of packets are plotted for every scheduling algorithm. We can see from these graphs that in all scheduling algorithms, the MRTs for type 1-1 are the best of all types. The reasons are that the M.R.R. (Mean Resource Requirement, or Mean Overall Service Time) for type 1-1 is smaller than the M.R.R.s for the other types (see Table 1) and that the preferential treatments listed as PT1, PT2, PT3 are applied to type 1-1, except for the following 

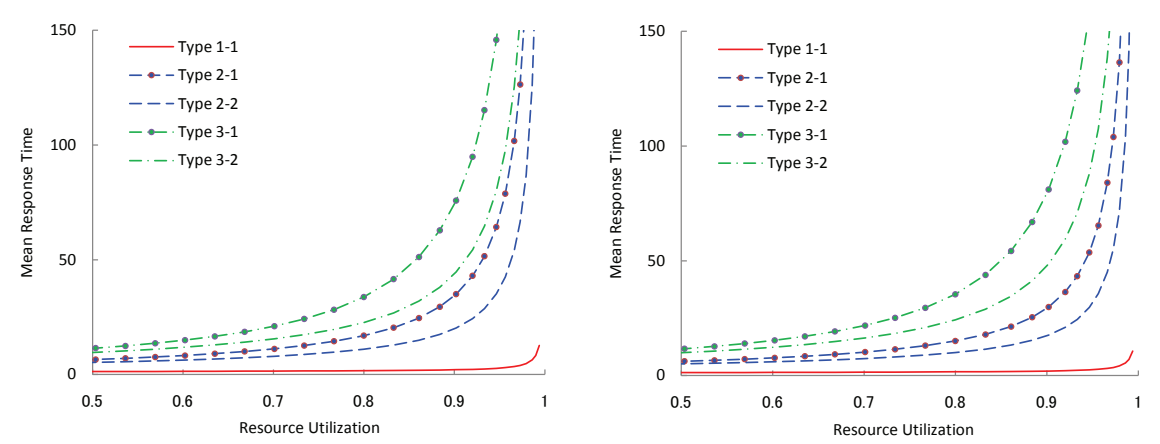

Fig. 4 MRTs for A-3 (left) and MRTs for A-4 (right)
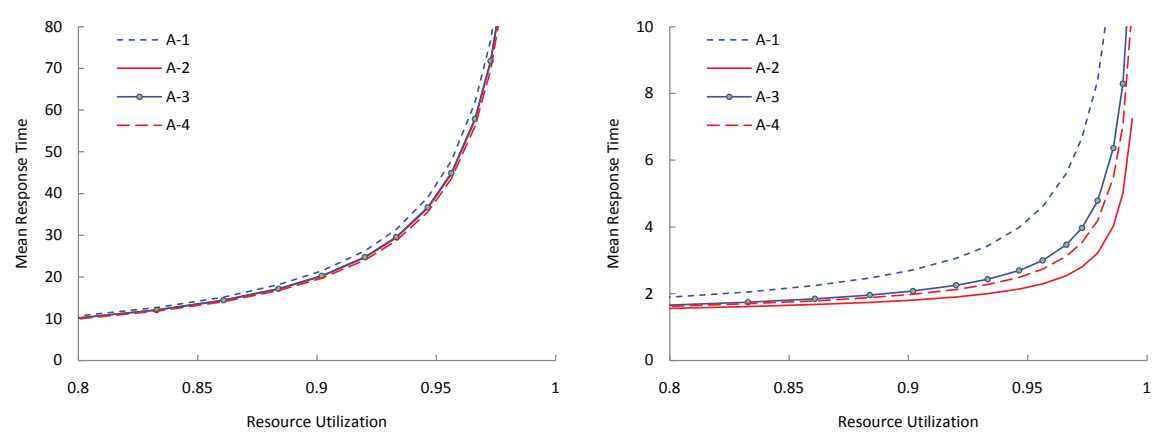

Fig. 5 MRTs averaged over all categories (left) and MRTs for category 1 (right)
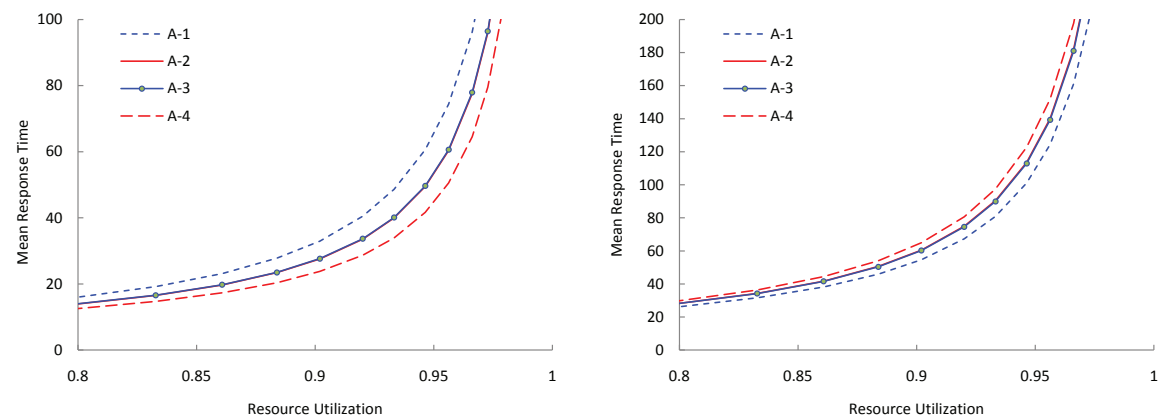

Fig. 6 MRTs for category 2 (left) and MRTs for category 3 (right)

case. Because PT1 is not applied to type 1-1 in algorithm A-1, the MRT for type 1-1 in A-1 is somewhat worse than its MRTs in the other algorithms. Further the MRTs for category 2 are almost better than those for category 3, because the M.R.R.s for category 2 are less than those for category 3 and PT1 is applied to category 2, except for the following case. Because PT1 is not applied to any type in A-1, the differences in the MRTs between categories 2 and 3 in A-1 are relatively small. Since the M.Q.S.s for type 2-1 and type 3-1 are equal, total number of the resource allocation (or service stages) for type 2-1 is less than that for type 3-1, and hence every MRT for type 2- 
1 is less than that for type 3-1 even if all the other conditions are equal. The same explanations are applicable to types 2-2 and 3-2.

In Figs. 5 and 6, the MRTs for all algorithms are plotted for every category and those averaged over all categories are plotted (the left graph in Fig. 5). From Fig. 5, we see that the overall MRTs for all algorithms are close, but for category 1 the MRT for A-2 is the best of those for all algorithms. The reason may be that between two consecutive allocations of the resource to type 1-1, only one other type is allocated the resource in algorithm A-2, whereas two or more other types are allocated the resource in the other algorithms. For categories 2 and 3, the MRTs in algorithms A-2 and A-3 are very close (i.e., the two curves for A-2 and A-3 in every graph in Fig. 6 are almost overlapped). The reason is in the similar resource allocation rules for categories 2 and 3 in these algorithms. However for category 1 we can see that the MRT in A-2 is better than the MRT in A-3. Hence we may conclude in this case that the algorithm A-2 is better than the algorithm A-3.

\section{Conclusions}

We have considered the multiclass feedback queues where the server visits the customer groups according to a Markov chain. There are multiple classes of customers in each group that are served in one of the following service orders: the gated FCFS, the gated priority, the exhaustive FCFS, and the exhaustive priority.

The functional computation method is used to analyze the mean sojourn times of all classes of customers spent at all stages of services. It can be shown that the expected values of the performance measures conditioned on the system state have the linear functional expressions. The first conception of the analysis of these queues was given in Hirayama (2009b). Although we have analyzed the model without switchover times, it may be possible to analyze the model with switchover times by a manner similar to that in Hirayama (2005) for the cyclic polling systems with switchover times.

Then our model is applied to the analysis of packet scheduling algorithms with five types (or three categories) of traffic. The important traffic can be preferentially treated by allocating the resource more often than the other traffic by arranging the polling probabilities etc. We consider four scheduling algorithms with different polling probabilities and service rules. The mean response times for all types of traffic in all scheduling algorithms are calculated and compared.

By using our queueing models, we can construct various types of composite scheduling algorithms that can approximate the actual network structures with many data sources, many traffic types and various QoS requirements. We can treat not only polling algorithms but also priority algorithms (Hirayama, 2003, 2010). Hence our methodology may enhance capabilities of the queueing models in performance evaluation of computer communication networks.

\section{Appendix: Detailed expressions for the coefficients in the propositions}

In this section, we give the detailed expressions for the coefficients in the propositions. Each subsection in this section corresponds to each proposition. Because the coefficients in each subsection (or proposition) use those in its previous subsections, we need to 
refer these subsections in order to calculate the coefficients. Further some elementary constants are quoted from the main sections.

We define the constant commonly used in this section. For $(j, \beta) \in \mathcal{S}$,

$$
\begin{aligned}
\mathbf{e}^{j, \beta} & =(0,0, \ldots, 0, \underbrace{1}_{(j, \beta)^{t h}}, 0,0, \ldots, 0) \in \mathcal{R}^{1 \times J_{c}}, \\
\overline{\mathbf{I}}^{j} & =\operatorname{diag}(1,1, \ldots, 1, \underbrace{0}_{(j, 1)^{t h}}, 0, \ldots, \underbrace{0}_{\left(j, L_{j}\right)^{t h}}, 1,1, \ldots, 1) \in \mathcal{R}^{J_{c} \times J_{c}} .
\end{aligned}
$$

9.1 The coefficients in Proposition 1

We define the following constants used in Proposition 1.

$$
\begin{aligned}
& \mathbf{h}_{10}^{0}(\cdot) \in \mathcal{R}^{2 J_{c} \times 1}, \varphi^{0}(\cdot) \in \mathcal{R}^{2 \times 1}, \mathbf{h}_{00}^{0}(\cdot) \in \mathcal{R}^{2 J_{c} \times 1} \\
& \mathbf{U}_{1}(\cdot) \in \mathcal{R}^{2 J_{c} \times 2 J_{c}}, \boldsymbol{v}_{0}(\cdot) \in \mathcal{R}^{2 \times 2 J_{c}}, \mathbf{U}_{0}(\cdot) \in \mathcal{R}^{2 J_{c} \times 2 J_{c}}, \mathbf{u}_{0}(\cdot) \in \mathcal{R}^{1 \times 2 J_{c}}
\end{aligned}
$$

We first show for reference the constants defined in the main sections. The following quantities are used to calculate the constants $\mathbf{h}_{10}^{0}(\cdot), \varphi^{0}(\cdot), \mathbf{h}_{00}^{0}(\cdot)$.

$$
\begin{aligned}
& \bar{T}_{\kappa_{0}, a_{0}}^{L_{\kappa_{0}}}(r)=r+\sum_{\beta=1}^{L_{\kappa_{0}}} p_{\kappa_{0}, a_{0}, \kappa_{0}, \beta} \bar{T}_{\kappa_{0}, \beta}^{L_{\kappa_{0}},}, \\
& \varrho_{\kappa_{0}, L_{\kappa_{0}}}^{+}=\sum_{\beta=1}^{L_{\kappa_{0}}} \lambda_{\kappa_{0}, \beta} \bar{T}_{\kappa_{0}, \beta}^{L_{\kappa_{0}},},
\end{aligned}
$$

where $\bar{T}_{\kappa_{0}, \beta}^{L_{\kappa_{0}}}$ is defined in (13). Then let

$$
\begin{array}{r}
\bar{N}_{i, k, \gamma}^{0}= \begin{cases}\lambda_{k, \gamma}, & i \in \mathcal{H}_{g}, \\
\lambda_{k, \gamma}+\sum_{\beta=1}^{L_{i}} \lambda_{i, \beta} \bar{N}_{i, \beta, k, \gamma}, & k \neq i \in \mathcal{H}_{e}, \\
0, & k=i \in \mathcal{H}_{e},\end{cases} \\
\bar{N}_{i, \alpha, k, \gamma}^{1}= \begin{cases}p_{i, \alpha, k, \gamma}, & i \in \mathcal{H}_{g}, \\
p_{i, \alpha, k, \gamma}+\sum_{\beta=1}^{L_{i}} p_{i, \alpha, i, \beta} \bar{N}_{i, \beta, k, \gamma}, & k \neq i \in \mathcal{H}_{e}, \\
0, & k=i \in \mathcal{H}_{e},\end{cases}
\end{array}
$$

where $\bar{N}_{i, \beta, k, \gamma}$ is defined in (14). Then the constant defined in (15) is given by

$$
\bar{N}_{\kappa_{0}, a_{0}, m, \gamma}(r)=r \bar{N}_{\kappa_{0}, m, \gamma}^{0}+\bar{N}_{\kappa_{0}, a_{0}, m, \gamma}^{1}
$$

which is used to calculate the constant $\boldsymbol{v}_{0}(\cdot)$.

Then we define

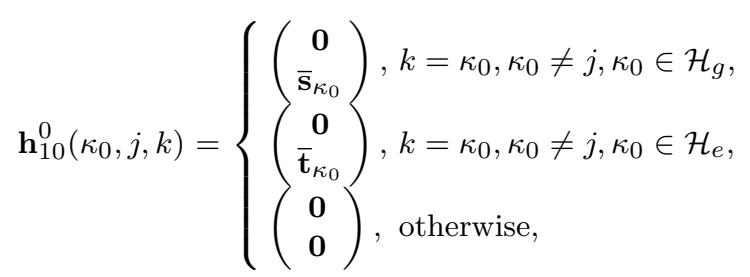




$$
\begin{aligned}
\varphi^{0}\left(\kappa_{0}, a_{0}, j, k\right)= \begin{cases}\left(\begin{array}{l}
1 \\
0
\end{array}\right), & k=\kappa_{0}, \kappa_{0} \in \mathcal{H}_{g}, \\
\frac{1}{1-\varrho_{\kappa_{0}, L_{\kappa_{0}}}^{+}}\left(\sum_{\beta=1}^{L_{\kappa_{0}} p_{\kappa_{0}, a_{0}, \kappa_{0}, \beta} \bar{T}_{\kappa_{0}, \beta}^{L_{\kappa_{0}}}}\right), \begin{array}{l}
k=\kappa_{0}, \quad \kappa_{0} \in \mathcal{H}_{e}, \\
\kappa_{0} \neq j,
\end{array} & \text { otherwise, } \\
\left(\begin{array}{c}
0 \\
0
\end{array}\right), & \left\{\begin{array}{l}
\left(\begin{array}{c}
\overline{\mathbf{s}}_{\kappa_{0}} \\
\mathbf{0}
\end{array}\right), k=\kappa_{0}, \kappa_{0} \in \mathcal{H}_{g}, \\
\mathbf{h}_{00}^{0}\left(\kappa_{0}, j, k\right)=\left\{\begin{array}{c}
\overline{\mathbf{t}}_{\kappa_{0}} \\
\mathbf{0}
\end{array}\right), k=\kappa_{0}, \kappa_{0} \neq j, \kappa_{0} \in \mathcal{H}_{e}, \\
\mathbf{0} \\
\mathbf{0}
\end{array}\right), \text { otherwise, }\end{cases}
\end{aligned}
$$

where $\mathbf{0} \in \mathcal{R}^{J_{c} \times 1}$ is a zero vector and where

$$
\begin{aligned}
& \overline{\mathbf{s}}_{\kappa_{0}}=\left(0, \ldots, 0, E\left[S_{\kappa_{0}, 1}\right], \ldots, E\left[S_{\kappa_{0}, L_{\kappa_{0}}}\right], 0, \ldots, 0\right)^{\prime} \in \mathcal{R}^{J_{c} \times 1}, \\
& \overline{\mathbf{t}}_{\kappa_{0}}=\frac{1}{1-\varrho_{\kappa_{0}, L_{\kappa_{0}}}^{+}}\left(0, \ldots, 0, \bar{T}_{\kappa_{0}, 1}^{\left.L_{\kappa_{0}}, \ldots, \bar{T}_{\kappa_{0}, L_{\kappa_{0}}}^{L_{\kappa_{0}}}, 0, \ldots, 0\right)^{\prime} \in \mathcal{R}^{J_{c} \times 1} .}\right.
\end{aligned}
$$

Further we define

$$
\begin{aligned}
& \mathbf{U}_{1}\left(\kappa_{0}\right)=\left(\begin{array}{lc}
\mathbf{O} & \mathbf{O} \\
\mathbf{O} & \mathbf{U}_{n}\left(\kappa_{0}\right)
\end{array}\right), \quad \boldsymbol{v}_{0}\left(\kappa_{0}, a_{0}\right)= \begin{cases}\left(\begin{array}{cc}
\mathbf{0} & \overline{\mathbf{N}}_{\kappa_{0}}^{0} \\
\mathbf{0} & \mathbf{N}_{\kappa_{0}, a_{0}}^{1}
\end{array}\right),\left(\left(\kappa_{0}, a_{0}\right) \in \mathcal{S}\right), \\
\mathbf{O}_{v}, & \left(\left(\kappa_{0}, a_{0}\right)=(0,0)\right),\end{cases} \\
& \mathbf{U}_{0}\left(\kappa_{0}\right)= \begin{cases}\left(\begin{array}{cc}
\mathbf{O} & \mathbf{U}_{g}\left(\kappa_{0}\right) \\
\mathbf{O} & \mathbf{I} \\
\mathbf{O} & \mathbf{U}_{g}\left(\kappa_{0}\right) \\
\mathbf{O} & \overline{\mathbf{I}}^{\kappa_{0}}
\end{array}\right), & \left(\kappa_{0} \in \mathcal{H}_{g}\right), \\
\mathbf{O}_{U}, & \left(\kappa_{0} \in \mathcal{H}_{e}\right), \\
\left(\kappa_{0}=0\right),\end{cases}
\end{aligned}
$$

$\mathbf{u}_{0}\left(\kappa_{0}, j, \beta\right)=\left(\mathbf{0}, u_{0 ; 1,1}\left(\kappa_{0}, j, \beta\right), \ldots, u_{0 ; J, L_{J}}\left(\kappa_{0}, j, \beta\right)\right)$,

where $\mathbf{I}, \mathbf{O} \in \mathcal{R}^{J_{c} \times J_{c}} ; \mathbf{O}_{v} \in \mathcal{R}^{2 \times 2 J_{c}} ; \mathbf{O}_{U} \in \mathcal{R}^{2 J_{c} \times 2 J_{c}} ; \mathbf{0} \in \mathcal{R}^{1 \times J_{c}}$ are an identity matrix and zero matrices, respectively, and where the constants $\mathbf{U}_{n}\left(\kappa_{0}\right), \mathbf{U}_{g}\left(\kappa_{0}\right) \in$ $\mathcal{R}^{J_{c} \times J_{c}} ; \overline{\mathbf{N}}_{\kappa_{0}, \alpha}, \overline{\mathbf{N}}_{\kappa_{0}}^{0}, \overline{\mathbf{N}}_{\kappa_{0}, a_{0}}^{1} \in \mathcal{R}^{1 \times J_{c}}$ and $u_{0 ; m, \gamma}(\cdot) \in \mathcal{R}$ are defined by

$$
\mathbf{U}_{n}\left(\kappa_{0}\right)=\left(\begin{array}{c}
\mathbf{e}^{1,1} \\
\vdots \\
\mathbf{e}^{\kappa_{0}-1, L_{\kappa_{0}-1}} \\
\overline{\mathbf{N}}_{\kappa_{0}, 1} \\
\vdots \\
\overline{\mathbf{N}}_{\kappa_{0}, L_{\kappa}} \\
\mathbf{e}^{\kappa_{0}+1,1} \\
\vdots \\
\mathbf{e}^{J, L_{J}}
\end{array}\right), \quad \mathbf{U}_{g}\left(\kappa_{0}\right)=\left(\begin{array}{c}
\mathbf{0} \\
\vdots \\
\mathbf{0} \\
\overline{\mathbf{N}}_{\kappa_{0}, 1} \\
\vdots \\
\overline{\mathbf{N}}_{\kappa_{0}, L_{\kappa_{0}}} \\
\mathbf{0} \\
\vdots \\
\mathbf{0}
\end{array}\right)
$$




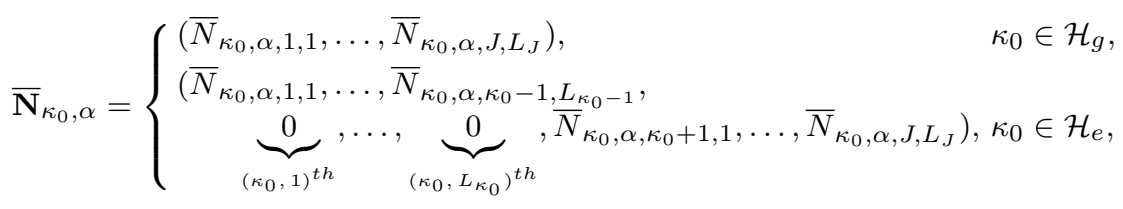

$$
\begin{aligned}
& \overline{\mathbf{N}}_{\kappa_{0}}^{0}= \begin{cases}\left(\bar{N}_{\kappa_{0}, 1,1}^{0}, \ldots, \bar{N}_{\kappa_{0}, J, L_{J}}^{0}\right), & \kappa_{0} \in \mathcal{H}_{g}, \\
\left(\bar{N}_{\kappa_{0}, 1,1}^{0}, \ldots, \bar{N}_{\kappa_{0}, \kappa_{0}-1, L_{\kappa_{0}-1}}^{0},\right. & \underbrace{0}_{\left(\kappa_{0}, 1\right)^{t h}}, \ldots, \underbrace{0}_{\left(\kappa_{0}, L_{\kappa_{0}}\right)^{t h}}, \bar{N}_{\kappa_{0}, \kappa_{0}+1,1}^{0}, \ldots, \bar{N}_{\kappa_{0}, J, L J}^{0}), \kappa_{0} \in \mathcal{H}_{e}\end{cases} \\
& \overline{\mathbf{N}}_{\kappa_{0}, a_{0}}^{1}= \begin{cases}\left(\bar{N}_{\kappa_{0}, a_{0}, 1,1}^{1}, \ldots, \bar{N}_{\kappa_{0}, a_{0}, J, L_{J}}^{1}\right), & \kappa_{0} \in \mathcal{H}_{g}, \\
(\bar{N}_{\kappa_{0}, a_{0}, 1,1}^{1}, \ldots, \bar{N}_{\kappa_{0}, a_{0}, \kappa_{0}-1, L_{\kappa_{0}-1},}^{1}, \underbrace{0}_{\left(\kappa_{0}, 1\right)^{t h}}, \ldots, \underbrace{0}_{\left.\kappa_{0}, L_{\kappa_{0}}\right)^{t h}}, \bar{N}_{\kappa_{0}, a_{0}, \kappa_{0}+1,1}^{1}, \ldots, \bar{N}_{\kappa_{0}, a_{0}, J, L J}^{1}), \kappa_{0} \in \mathcal{H}_{e}, & \end{cases} \\
& u_{0 ; m, \gamma}\left(\kappa_{0}, j, \beta\right)= \begin{cases}\mathbf{1}_{m, \gamma}(j, \beta), & \kappa_{0} \in \mathcal{H}_{g} \text { or } \kappa_{0}=0 \\
\mathbf{1}_{m, \gamma}(j, \beta)+\sum_{\alpha=1}^{L_{\kappa_{0}}} \mathbf{1}_{\kappa_{0}, \alpha}(j, \beta) \bar{N}_{\kappa_{0}, \alpha, m, \gamma}, & m \neq \kappa_{0}, \kappa_{0} \in \mathcal{H}_{e} \\
0, & m=\kappa_{0}, \kappa_{0} \in \mathcal{H}_{e}\end{cases}
\end{aligned}
$$

9.2 The coefficients in Proposition 2

We define the following constants used in Proposition 2.

$$
\mathbf{B}_{1}^{j}(\cdot) \in \mathcal{R}^{2 J_{c} \times J_{c}} ; \mathbf{c}^{j}(\cdot) \in \mathcal{R}^{2 \times J_{c}} ; \mathbf{B}_{0}^{j}(\cdot) \in \mathcal{R}^{2 J_{c} \times J_{c}} ; \mathbf{b}_{0}^{j, \beta}(\cdot) \in \mathcal{R}^{1 \times J_{c}} .
$$

Let us define the constant $\mathbf{B}_{1}^{j}(\cdot) \in \mathcal{R}^{2 J_{c} \times J_{c}}$ that satisfy the following linear equation.

$$
\mathbf{B}_{1}^{j}\left(\kappa_{0}\right)= \begin{cases}\left(\begin{array}{c}
\mathbf{O} \\
\mathbf{I}
\end{array}\right), & \kappa_{0}=j, \\
\mathbf{U}_{1}\left(\kappa_{0}\right)\left\{\sum_{\kappa_{1} \in \Pi \backslash\{j\}} \hat{p}_{\kappa_{0}, \kappa_{1}} \mathbf{B}_{1}^{j}\left(\kappa_{1}\right)+\hat{p}_{\kappa_{0}, j}\left(\begin{array}{c}
\mathbf{O} \\
\mathbf{I}
\end{array}\right)\right\}, \kappa_{0} \neq j,\end{cases}
$$

for $\kappa_{0}, j \in \Pi$ where $\mathbf{O}, \mathbf{I} \in \mathcal{R}^{J_{c} \times J_{c}}$ are a zero matrix and an identity matrix, respectively. Further we define the following constants $\mathbf{c}^{j}(\cdot) \in \mathcal{R}^{2 \times J_{c}}, \mathbf{B}_{0}^{j}(\cdot) \in \mathcal{R}^{2 J_{c} \times J_{c}}$ and $\mathbf{b}_{0}^{j, \beta}(\cdot) \in \mathcal{R}^{1 \times J_{c}}$.

$$
\begin{aligned}
& \mathbf{c}^{j}\left(\kappa_{0}, a_{0}\right)= \begin{cases}\sum_{\kappa_{1}=1}^{J} \hat{p}_{\kappa_{0}, \kappa_{1}} \boldsymbol{v}_{0}\left(\kappa_{0}, a_{0}\right) \mathbf{B}_{1}^{j}\left(\kappa_{1}\right), & \kappa_{0} \neq j \text { or } \kappa_{0}=j \in \mathcal{H}_{g}, \\
\mathbf{0}, & \kappa_{0}=j \in \mathcal{H}_{e},\end{cases} \\
& \mathbf{B}_{0}^{j}\left(\kappa_{0}\right)= \begin{cases}\sum_{\kappa_{1}=1}^{J} \hat{p}_{\kappa_{0}, \kappa_{1}} \mathbf{U}_{0}\left(\kappa_{0}\right) \mathbf{B}_{1}^{j}\left(\kappa_{1}\right), & \kappa_{0} \neq j \text { or } \kappa_{0}=j \in \mathcal{H}_{g}, \\
\left(\begin{array}{c}
\mathbf{O} \\
\mathbf{I}
\end{array}\right), & \kappa_{0}=j \in \mathcal{H}_{e},\end{cases} \\
& \mathbf{b}_{0}^{j, \beta}\left(\kappa_{0}\right)= \begin{cases}\sum_{\kappa_{1}=1}^{J} \hat{p}_{\kappa_{0}, \kappa_{1}} \mathbf{u}_{0}\left(\kappa_{0}, j, \beta\right) \mathbf{B}_{1}^{j}\left(\kappa_{1}\right), & \kappa_{0} \neq j \text { or } \kappa_{0}=j \in \mathcal{H}_{g}, \\
\mathbf{0}, & \kappa_{0}=j \in \mathcal{H}_{e},\end{cases}
\end{aligned}
$$

for $\left(\kappa_{0}, a_{0}\right),(j, \beta) \in \mathcal{S}$. For $\left(\kappa_{0}, a_{0}\right)=(0,0)$ and $(j, \beta) \in \mathcal{S}$, we define

$$
\mathbf{B}_{1}^{j}(0)=\mathbf{O}, \quad \mathbf{c}^{j}(0,0)=\mathbf{0}, \quad \mathbf{B}_{0}^{j}(0)=\mathbf{O}, \quad \mathbf{b}_{0}^{j, \beta}(0)=\mathbf{e}^{j, \beta} .
$$


9.3 The coefficients in Proposition 3

We define the following constants used in Proposition 3.

$$
\begin{aligned}
& \mathbf{h}_{10}^{j}(\cdot) \in \mathcal{R}^{2 J_{c} \times 1} ; \\
& \boldsymbol{\varphi}^{j}(\cdot), \boldsymbol{\eta}^{j, \beta}(\cdot) \in \mathcal{R}^{2 \times 1} ; \mathbf{h}_{00}^{j}(\cdot), \mathbf{f}^{j, \beta}(\cdot) \in \mathcal{R}^{2 J_{c} \times 1} ; h_{01}^{j, \beta}(\cdot), f^{j, \beta}(\cdot) \in \mathcal{R} .
\end{aligned}
$$

Let us define constants $\mathbf{h}_{10}^{j}\left(\kappa_{0}, k\right) \in \mathcal{R}^{2 J_{c} \times 1}$ that satisfy the following linear equation:

$$
\mathbf{h}_{10}^{j}\left(\kappa_{0}, k\right)=\left\{\begin{array}{cc}
\mathbf{h}_{10}^{0}\left(\kappa_{0}, j, k\right)+\mathbf{U}_{1}\left(\kappa_{0}\right) \sum_{\kappa_{1} \in \Pi \backslash\{j\}} \hat{p}_{\kappa_{0} \kappa_{1}} \mathbf{h}_{10}^{j}\left(\kappa_{1}, k\right), \\
\kappa_{0} \neq j, \kappa_{0} \in \Pi, \\
\mathbf{0}, \quad \kappa_{0}=j \text { or } \kappa_{0}=0,
\end{array}\right.
$$

for $\kappa_{0} \in \Pi \cup\{0\} ; j, k \in \Pi$. Further for $\left(\kappa_{0}, a_{0}\right) \in \mathcal{S} \cup\{0,0\},(j, \beta) \in \mathcal{S}$ and $k \in \Pi$, let

$$
\begin{aligned}
\boldsymbol{\varphi}^{j}\left(\kappa_{0}, a_{0}, k\right) & = \begin{cases}\boldsymbol{\varphi}^{0}\left(\kappa_{0}, a_{0}, j, k\right)+\boldsymbol{v}_{0}\left(\kappa_{0}, a_{0}\right) \sum_{\kappa_{1} \in \Pi \backslash\{j\}} \hat{p}_{\kappa_{0} \kappa_{1}} \mathbf{h}_{10}^{j}\left(\kappa_{1}, k\right), & \text { case 1, } \\
\mathbf{0}, & \text { case 2, }\end{cases} \\
\mathbf{h}_{00}^{j}\left(\kappa_{0}, k\right) & = \begin{cases}\mathbf{h}_{00}^{0}\left(\kappa_{0}, j, k\right)+\mathbf{U}_{0}\left(\kappa_{0}\right) \sum_{\kappa_{1} \in \Pi \backslash\{j\}} \hat{p}_{\kappa_{0} \kappa_{1}} \mathbf{h}_{10}^{j}\left(\kappa_{1}, k\right), & \text { case 1, } \\
\mathbf{0}, & \text { case 2, }\end{cases} \\
h_{01}^{j, \beta}\left(\kappa_{0}, k\right) & = \begin{cases}\mathbf{u}_{0}\left(\kappa_{0}, j, \beta\right) \sum_{\kappa_{1} \in \Pi \backslash\{j\}} \hat{p}_{\kappa_{0} \kappa_{1}} \mathbf{h}_{10}^{j}\left(\kappa_{1}, k\right), & \text { case 1, } \\
0, & \text { case 2, }\end{cases}
\end{aligned}
$$

where "case 1 " denotes $\left(\kappa_{0} \neq j, \kappa_{0} \in \Pi\right)$ or $\left(j \in \mathcal{H}_{g}\right)$; and "case 2 " denotes $\left(\kappa_{0}=j \in\right.$ $\left.\mathcal{H}_{e}\right)$ or $\left(\kappa_{0}=0\right)$.

Further for $\left(\kappa_{0}, a_{0}\right) \in \mathcal{S}$, we define

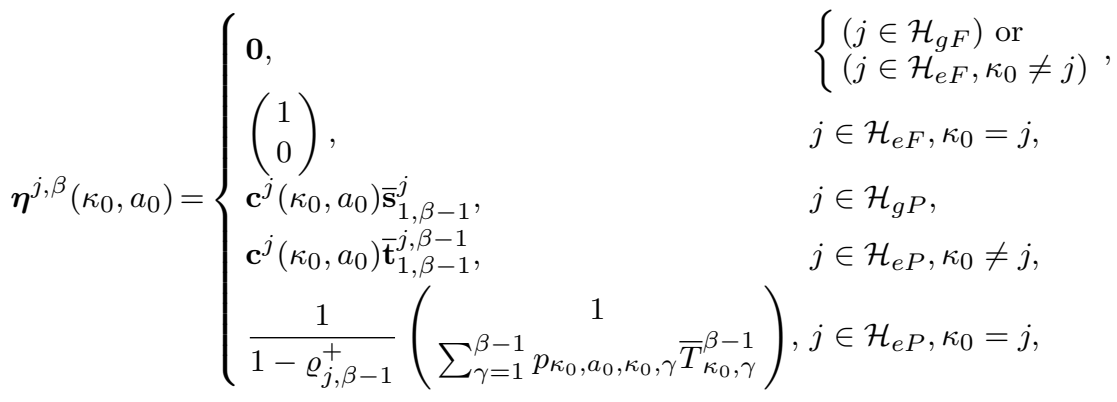

$$
\begin{aligned}
& \mathbf{f}^{j, \beta}\left(\kappa_{0}\right)= \begin{cases}\left(\begin{array}{c}
\mathbf{0} \\
\overline{\mathbf{s}}_{1, L_{j}}^{j}
\end{array}\right), & \left\{\begin{array}{l}
\left(j \in \mathcal{H}_{g F}\right) \text { or } \\
\left(j \in \mathcal{H}_{e F} \& \kappa_{0} \neq j\right)
\end{array}\right. \\
\left(\begin{array}{c}
\overline{\mathbf{s}}_{1, L_{j}}^{j} \\
\mathbf{0}
\end{array}\right), & j \in \mathcal{H}_{e F}, \kappa_{0}=j, \\
\mathbf{B}_{0}^{j}\left(\kappa_{0}\right) \overline{\mathbf{s}}_{1, \beta-1}^{j}+\left(\begin{array}{c}
\mathbf{0} \\
\overline{\mathbf{s}}_{\beta, \beta}^{j}
\end{array}\right), & j \in \mathcal{H}_{g P}, \\
\mathbf{B}_{0}^{j}\left(\kappa_{0}\right) \overline{\mathbf{t}}_{1, \beta-1}^{j, \beta-1}+\left(\begin{array}{c}
\mathbf{0} \\
\overline{\mathbf{t}}_{\beta, \beta}^{j, \beta}
\end{array}\right), & j \in \mathcal{H}_{e P}, \kappa_{0} \neq j, \\
\left(\begin{array}{c}
\overline{\mathbf{t}}_{1, \beta}^{j, \beta-1} \\
\mathbf{0}
\end{array}\right), & j \in \mathcal{H}_{e P}, \kappa_{0}=j,\end{cases}
\end{aligned}
$$




$$
f^{j, \beta}\left(\kappa_{0}\right)= \begin{cases}E\left[S_{j, \beta}\right], & \left\{\begin{array}{l}
\left(j \in \mathcal{H}_{g F}\right) \text { or } \\
\left(j \in \mathcal{H}_{e F}, \kappa_{0} \neq j\right)
\end{array}\right. \\
E\left[S_{j, \beta}\right], & j \in \mathcal{H}_{e F}, \kappa_{0}=j, \\
\mathbf{b}_{0}^{j, \beta}\left(\kappa_{0}\right) \overline{\mathbf{s}}_{1, \beta-1}^{j}+E\left[S_{j, \beta}\right], & j \in \mathcal{H}_{g P}, \\
\mathbf{b}_{0}^{j, \beta}\left(\kappa_{0}\right) \overline{\mathbf{t}}_{1, \beta-1}^{j, \beta-1}+E\left[S_{j, \beta}\right], & j \in \mathcal{H}_{e P}, \kappa_{0} \neq j, \\
E\left[S_{j, \beta}\right], & j \in \mathcal{H}_{e P}, \kappa_{0}=j,\end{cases}
$$

where we define vectors $\overline{\mathbf{s}}_{\alpha, \gamma}^{j}, \overline{\mathbf{t}}_{\alpha, \gamma}^{j, \beta-1} \in \mathcal{R}^{J_{c} \times 1}$ as

$$
\begin{aligned}
\overline{\mathbf{s}}_{\alpha, \gamma}^{j} & =(0, \ldots, 0, \underbrace{E\left[S_{j, \alpha}\right]}_{(j, \alpha)^{t h} \text { place }}, E\left[S_{j, \alpha+1}\right], \ldots, \underbrace{E\left[S_{j, \gamma}\right]}_{(j, \gamma)^{t h} \text { place }}, 0, \ldots, 0)^{\prime}, \\
\overline{\mathbf{t}}_{\alpha, \gamma}^{j, \beta-1} & =\frac{1}{1-\varrho_{j, \beta-1}^{+}}(0, \ldots, 0, \underbrace{\bar{T}_{j, \alpha}^{\beta-1}}_{(j, \alpha)^{t h} \text { place }}, \bar{T}_{j, \alpha+1}^{\beta-1}, \ldots, \underbrace{\bar{T}_{j, \gamma}^{\beta-1}}_{(j, \gamma)^{t h} \text { place }}, 0, \ldots, 0)^{\prime},
\end{aligned}
$$

for $j \in \Pi$ and $1 \leq \alpha, \beta, \gamma \leq L_{j},(\alpha \leq \gamma)$, and where $\bar{T}_{j, \alpha}^{\beta-1}, \varrho_{j, \beta-1}^{+}$are defined by (13) and the equation below it, respectively. For $\left(\kappa_{0}, a_{0}\right)=(0,0)$, we define

$$
\boldsymbol{\eta}^{j, \beta}(0,0)=\mathbf{0}, \quad \mathbf{f}^{j, \beta}(0)=\mathbf{0}, \quad f^{j, \beta}(0)=E\left[S_{j, \beta}\right] .
$$

9.4 The coefficients in Proposition 4

We define the following constants used in Proposition 4.

$$
\boldsymbol{v}^{j, \beta}(\cdot) \in \mathcal{R}^{2 \times 2 J_{c}} ; \mathbf{U}^{j, \beta}(\cdot) \in \mathcal{R}^{2 J_{c} \times 2 J_{c}} ; \mathbf{u}^{j, \beta}(\cdot) \in \mathcal{R}^{1 \times 2 J_{c}} .
$$

For simplicity, we divide each vector or matrix into two components with the same size as follows.

$$
\begin{aligned}
\boldsymbol{v}^{j, \beta}\left(\kappa_{0}, a_{0}\right) & =\left(\boldsymbol{v}_{g}^{j, \beta}\left(\kappa_{0}, a_{0}\right), \boldsymbol{v}_{n}^{j, \beta}\left(\kappa_{0}, a_{0}\right)\right), & & \left(\boldsymbol{v}_{g}^{j, \beta}(\cdot), \boldsymbol{v}_{n}^{j, \beta}(\cdot) \in \mathcal{R}^{2 \times J_{c}}\right), \\
\mathbf{U}^{j, \beta}\left(\kappa_{0}\right) & =\left(\mathbf{U}_{g}^{j, \beta}\left(\kappa_{0}\right), \mathbf{U}_{n}^{j, \beta}\left(\kappa_{0}\right)\right), & & \left(\mathbf{U}_{g}^{j, \beta}(\cdot), \mathbf{U}_{n}^{j, \beta}(\cdot) \in \mathcal{R}^{2 J_{c} \times J_{c}}\right), \\
\mathbf{u}^{j, \beta}\left(\kappa_{0}\right) & =\left(\mathbf{u}_{g}^{j, \beta}\left(\kappa_{0}\right), \mathbf{u}_{n}^{j, \beta}\left(\kappa_{0}\right)\right), & & \left(\mathbf{u}_{g}^{j, \beta}(\cdot), \mathbf{u}_{n}^{j, \beta}(\cdot) \in \mathcal{R}^{1 \times J_{c}}\right) .
\end{aligned}
$$

Let us define constants commonly used in the following expressions.

$$
\begin{aligned}
& \mathbf{I}_{\alpha, \gamma}^{j}=\operatorname{diag}(0, \ldots, 0, \underbrace{1}_{(j, \alpha)^{t h}}, 1, \ldots, \underbrace{1}_{(j, \gamma)^{t h}}, 0, \ldots, 0) \in \mathcal{R}^{J_{c} \times J_{c}}, \\
& \boldsymbol{\lambda}=\left(\lambda_{j, \beta}:(j, \beta) \in \mathcal{S}\right) \in \mathcal{R}^{1 \times J_{c}}, \quad \mathbf{p}_{j, a}=\left(p_{j, a, k, \gamma}:(k, \gamma) \in \mathcal{S}\right) \in \mathcal{R}^{1 \times J_{c}} .
\end{aligned}
$$

Gated group $j\left(j \in \mathcal{H}_{g}\right)$

For $\left(\kappa_{0}, a_{0}\right) \in \mathcal{S}$,

$$
\begin{aligned}
& \boldsymbol{v}_{g}^{j, \beta}\left(\kappa_{0}, a_{0}\right)= \begin{cases}\mathbf{c}^{j}\left(\kappa_{0}, a_{0}\right) \mathbf{I}_{1, L_{j}}^{j}, j \in \mathcal{H}_{g F}, \\
\mathbf{c}^{j}\left(\kappa_{0}, a_{0}\right) \mathbf{I}_{\beta, L_{j}}^{j}, j \in \mathcal{H}_{g P},\end{cases} \\
& \boldsymbol{v}_{n}^{j, \beta}\left(\kappa_{0}, a_{0}\right)= \begin{cases}\mathbf{c}^{j}\left(\kappa_{0}, a_{0}\right) \overline{\mathbf{I}}^{j}+\boldsymbol{\eta}^{j, \beta}\left(\kappa_{0}, a_{0}\right) \boldsymbol{\lambda}, \\
\mathbf{c}^{j}\left(\kappa_{0}, a_{0}\right)\left(\overline{\mathbf{I}}^{j}+\mathbf{I}_{1, \beta-1}^{j} \mathbf{P}\right)+\boldsymbol{\eta}^{j, \beta}\left(\kappa_{0}, a_{0}\right) \boldsymbol{\lambda}, j \in \mathcal{H}_{g P},\end{cases}
\end{aligned}
$$




$$
\begin{aligned}
& \mathbf{U}_{g}^{j, \beta}\left(\kappa_{0}\right)=\left\{\begin{array}{l}
\left\{\mathbf{B}_{0}^{j}\left(\kappa_{0}\right)-\left(\begin{array}{c}
\mathbf{O} \\
\mathbf{I}
\end{array}\right)\right\} \mathbf{I}_{1, L_{j}}^{j}, \quad j \in \mathcal{H}_{g F}, \\
\left\{\mathbf{B}_{0}^{j}\left(\kappa_{0}\right)-\left(\begin{array}{c}
\mathbf{O} \\
\mathbf{I}_{\beta, \beta}^{j}
\end{array}\right)\right\} \mathbf{I}_{\beta, L_{j}}^{j}, j \in \mathcal{H}_{g P},
\end{array}\right. \\
& \mathbf{U}_{n}^{j, \beta}\left(\kappa_{0}\right)= \begin{cases}\mathbf{B}_{0}^{j}\left(\kappa_{0}\right) \overline{\mathbf{I}}^{j}+\mathbf{f}^{j, \beta}\left(\kappa_{0}\right) \boldsymbol{\lambda}+\left(\begin{array}{c}
\mathbf{O} \\
\mathbf{I}_{1, L_{j}}^{j} \mathbf{P}
\end{array}\right), & j \in \mathcal{H}_{g F}, \\
\mathbf{B}_{0}^{j}\left(\kappa_{0}\right)\left(\overline{\mathbf{I}}^{j}+\mathbf{I}_{1, \beta-1}^{j} \mathbf{P}\right)+\mathbf{f}^{j, \beta}\left(\kappa_{0}\right) \boldsymbol{\lambda}+\left(\begin{array}{c}
\mathbf{O} \\
\mathbf{I}_{\beta, \beta}^{j} \mathbf{P}
\end{array}\right), j \in \mathcal{H}_{g P},\end{cases}
\end{aligned}
$$

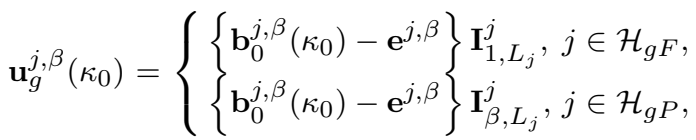

$$
\begin{aligned}
& \mathbf{u}_{n}^{j, \beta}\left(\kappa_{0}\right)=\left\{\begin{array}{lr}
\mathbf{b}_{0}^{j, \beta}\left(\kappa_{0}\right) \overline{\mathbf{I}}^{j}+f^{j, \beta}\left(\kappa_{0}\right) \boldsymbol{\lambda}, & j \in \mathcal{H}_{g F}, \\
\mathbf{b}_{0}^{j, \beta}\left(\kappa_{0}\right)\left(\overline{\mathbf{I}}^{j}+\mathbf{I}_{1, \beta-1}^{j} \mathbf{P}\right)+f^{j, \beta}\left(\kappa_{0}\right) \boldsymbol{\lambda}, & j \in \mathcal{H}_{g P} .
\end{array}\right.
\end{aligned}
$$

For $\left(\kappa_{0}, a_{0}\right)=(0,0)$,

$$
\begin{aligned}
& \boldsymbol{v}_{g}^{j, \beta}(0,0)=\mathbf{O}, \boldsymbol{v}_{n}^{j, \beta}(0,0)=\mathbf{O}, \mathbf{U}_{g}^{j, \beta}(0)=\mathbf{O}, \mathbf{U}_{n}^{j, \beta}(0)=\mathbf{O}, \\
& \mathbf{u}_{g}^{j, \beta}(0)=\mathbf{0}, \mathbf{u}_{n}^{j, \beta}(0)=E\left[S_{j, \beta}\right] \boldsymbol{\lambda} .
\end{aligned}
$$

Exhaustive FCFS group $j\left(j \in \mathcal{H}_{e F}\right)$

For $\left(\kappa_{0}, a_{0}\right) \in \mathcal{S}$,

$$
\begin{gathered}
\boldsymbol{v}_{g}^{j, \beta}\left(\kappa_{0}, a_{0}\right)=\left\{\begin{array}{l}
\left\{\boldsymbol{\eta}^{j, \beta}\left(\kappa_{0}, a_{0}\right) \boldsymbol{\lambda}+\left(\begin{array}{c}
\mathbf{0} \\
\mathbf{p}_{j, a_{0}}
\end{array}\right)\right\} \mathbf{I}_{1, L_{j}}^{j}, \kappa_{0}=j, \\
\left\{\mathbf{c}^{j}\left(\kappa_{0}, a_{0}\right)+\boldsymbol{\eta}^{j, \beta}\left(\kappa_{0}, a_{0}\right) \boldsymbol{\lambda}\right\} \mathbf{I}_{1, L_{j}}^{j}, \kappa_{0} \neq j,
\end{array}\right. \\
\boldsymbol{v}_{n}^{j, \beta}\left(\kappa_{0}, a_{0}\right)=\left\{\begin{array}{l}
\left\{\boldsymbol{\eta}^{j, \beta}\left(\kappa_{0}, a_{0}\right) \boldsymbol{\lambda}+\left(\begin{array}{c}
\mathbf{0} \\
\mathbf{p}_{j, a_{0}}
\end{array}\right)\right\} \mathbf{\mathbf { I }}^{j}, \kappa_{0}=j, \\
\left\{\mathbf{c}^{j}\left(\kappa_{0}, a_{0}\right)+\boldsymbol{\eta}^{j, \beta}\left(\kappa_{0}, a_{0}\right) \boldsymbol{\lambda}\right\} \overline{\mathbf{I}}^{j}, \kappa_{0} \neq j,
\end{array}\right. \\
\mathbf{U}_{g}^{j, \beta}\left(\kappa_{0}\right)=\left\{\begin{array}{l}
\left\{\mathbf{f}^{j, \beta}\left(\kappa_{0}\right) \boldsymbol{\lambda}+\left(\begin{array}{cc}
\mathbf{I}_{1, L_{j}}^{j} \mathbf{P} \\
\mathbf{O}
\end{array}\right)\right\} \mathbf{I}_{1, L_{j}}^{j}, \\
\left\{\mathbf{B}_{0}^{j}\left(\kappa_{0}\right)+\mathbf{f}^{j, \beta}\left(\kappa_{0}\right) \boldsymbol{\lambda}+\left(\begin{array}{cc}
\mathbf{O} \\
\mathbf{I}_{1, L_{j}}^{j} \mathbf{P}-\mathbf{I}
\end{array}\right)\right\} \mathbf{I}_{1, L_{j}}^{j}, \kappa_{0} \neq j,
\end{array}\right. \\
\mathbf{U}_{n}^{j, \beta}\left(\kappa_{0}\right)=\left\{\begin{array}{l}
\left\{\mathbf{f}^{j, \beta}\left(\kappa_{0}\right) \boldsymbol{\lambda}+\left(\begin{array}{c}
\mathbf{I}_{1, L_{j}}^{j} \mathbf{P} \\
\mathbf{I}
\end{array}\right)\right\} \overline{\mathbf{I}}^{j}, \\
\left\{\mathbf{B}_{0}^{j}\left(\kappa_{0}\right)+\mathbf{f}^{j, \beta}\left(\kappa_{0}\right) \boldsymbol{\lambda}+\left(\begin{array}{c}
\mathbf{O} \\
\mathbf{I}_{1, L_{j}}^{j} \mathbf{P}
\end{array}\right)\right\} \overline{\mathbf{I}}^{j}, \kappa_{0} \neq j,
\end{array}\right. \\
\mathbf{u}_{g}^{j, \beta}\left(\kappa_{0}\right)=\left\{\begin{array}{l}
\left\{f^{j, \beta}\left(\kappa_{0}\right) \boldsymbol{\lambda}\right\} \mathbf{I}_{1, L_{j}}^{j}, \\
\left\{\mathbf{b}_{0}^{j, \beta}\left(\kappa_{0}\right)-\mathbf{e}^{j, \beta}+f^{j, \beta}\left(\kappa_{0}\right) \boldsymbol{\lambda}\right\} \mathbf{I}_{1, L_{j}}^{j}, \kappa_{0} \neq j,
\end{array}\right. \\
\mathbf{u}_{n}^{j, \beta}\left(\kappa_{0}\right)=\left\{\begin{array}{l}
\left\{f^{j, \beta}\left(\kappa_{0}\right) \boldsymbol{\lambda}\right\} \overline{\mathbf{I}}^{j}, \\
\left\{\mathbf{b}_{0}^{j, \beta}\left(\kappa_{0}\right)+f^{j, \beta}\left(\kappa_{0}\right) \boldsymbol{\lambda}\right\} \overline{\mathbf{I}}^{j}, \kappa_{0} \neq j,
\end{array}\right.
\end{gathered}
$$


For $\left(\kappa_{0}, a_{0}\right)=(0,0)$,

$$
\begin{aligned}
& \boldsymbol{v}_{g}^{j, \beta}(0,0)=\mathbf{O}, \boldsymbol{v}_{n}^{j, \beta}(0,0)=\mathbf{O}, \mathbf{U}_{g}^{j, \beta}(0)=\mathbf{O}, \mathbf{U}_{n}^{j, \beta}(0)=\mathbf{O}, \\
& \mathbf{u}_{g}^{j, \beta}(0)=\left\{E\left[S_{j, \beta}\right] \boldsymbol{\lambda}\right\} \mathbf{I}_{1, L_{j}}^{j}, \mathbf{u}_{n}^{j, \beta}(0)=\left\{E\left[S_{j, \beta}\right] \boldsymbol{\lambda}\right\} \overline{\mathbf{I}}^{j} .
\end{aligned}
$$

Exhaustive priority group $j\left(j \in \mathcal{H}_{e P}\right)$

We define

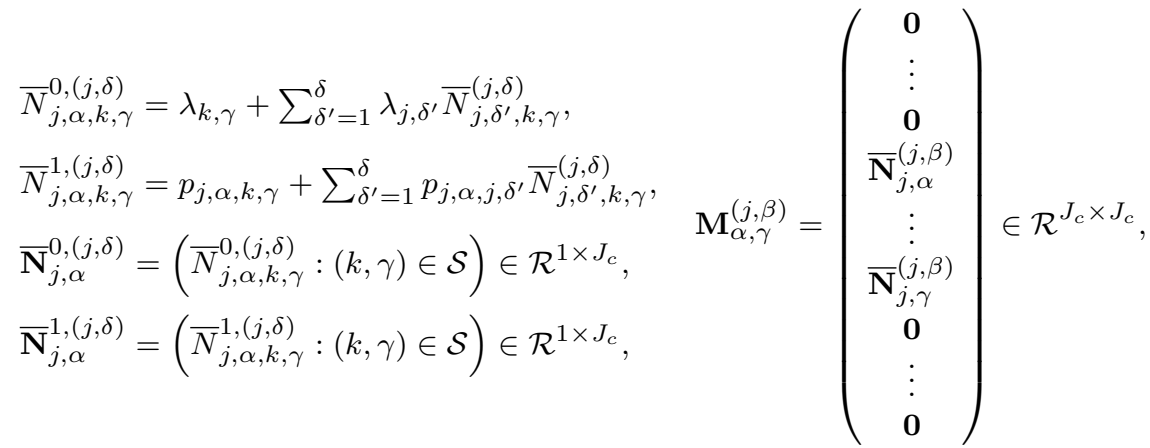

where $\mathbf{0} \in \mathcal{R}^{1 \times J_{c}}$ is a zero vector and $\overline{\mathbf{N}}_{j, \alpha}^{(j, \beta)}=\left(\bar{N}_{j, \alpha, k, \gamma}^{(j, \beta)}:(k, \gamma) \in \mathcal{S}\right) \in \mathcal{R}^{1 \times J_{c}}$ and $\bar{N}_{j, \alpha, k, \gamma}^{(j, \beta)}$ is defined in (33). Then the constant defined in (34) is given by

$$
\bar{N}_{j, \alpha, k, \gamma}^{(j, \delta)}(r)=r \bar{N}_{j, \alpha, k, \gamma}^{0,(j, \delta)}+\bar{N}_{j, \alpha, k, \gamma}^{1,(j, \delta)}
$$

which is used to calculate the constant $\boldsymbol{v}^{j, \beta}(\cdot)$.

For $\left(\kappa_{0}, a_{0}\right) \in \mathcal{S}$

$$
\begin{aligned}
& \boldsymbol{v}_{g}^{j, \beta}\left(\kappa_{0}, a_{0}\right)= \begin{cases}\left(\begin{array}{l}
\overline{\mathbf{N}}_{j, a_{0}}^{0,(j, \beta-1)} \\
\left.\overline{\mathbf{N}}_{j, a_{0}}^{1,(j, \beta-1)}\right) \mathbf{I}_{\beta, L_{j}}^{j},
\end{array}\right. & \kappa_{0}=j, \\
\mathbf{c}^{j}\left(\kappa_{0}, a_{0}\right)\left(\mathbf{I}+\mathbf{M}_{1, \beta-1}^{(j, \beta-1)}\right) \mathbf{I}_{\beta, L_{j}}^{j}, \kappa_{0} \neq j,\end{cases}
\end{aligned}
$$

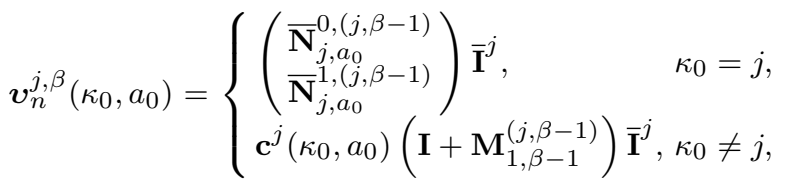

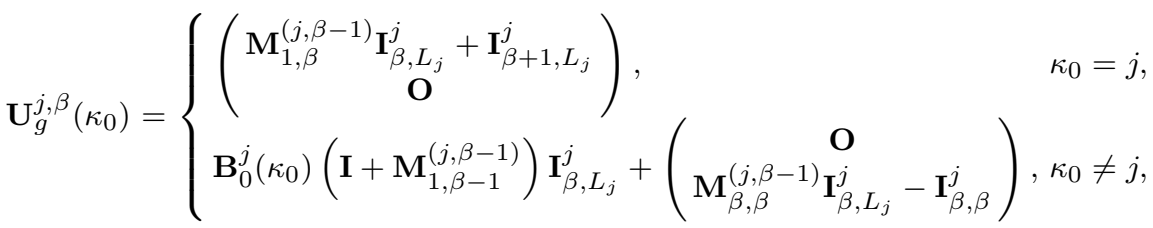

$$
\begin{aligned}
& \mathbf{U}_{n}^{j, \beta}\left(\kappa_{0}\right)= \begin{cases}\left(\begin{array}{c}
\mathbf{M}_{1, \beta}^{(j, \beta-1)} \\
\mathbf{I}
\end{array}\right) \overline{\mathbf{I}}^{j}, & \kappa_{0}=j, \\
\left\{\mathbf{B}_{0}^{j}\left(\kappa_{0}\right)\left(\mathbf{I}+\mathbf{M}_{1, \beta-1}^{(j, \beta-1)}\right)+\left(\begin{array}{c}
\mathbf{O} \\
\left.\left.\mathbf{M}_{\beta, \beta}^{(j, \beta-1)}\right)\right\} \overline{\mathbf{I}}^{j}, \kappa_{0} \neq j,
\end{array}\right.\right.\end{cases}
\end{aligned}
$$




$$
\begin{aligned}
& \mathbf{u}_{g}^{j, \beta}\left(\kappa_{0}\right)= \begin{cases}E\left[S_{j, \beta}\right] \mathbf{\lambda} \mathbf{I}_{1, L_{j}}^{j}, & \kappa_{0}=j, \\
\mathbf{b}_{0}^{j, \beta}\left(\kappa_{0}\right)\left(\mathbf{I}+\mathbf{M}_{1, \beta-1}^{(j, \beta-1)}\right) \mathbf{I}_{\beta, L_{j}}^{j}-\mathbf{e}^{j, \beta}+E\left[S_{j, \beta}\right] \boldsymbol{\lambda} \mathbf{I}_{1, L_{j}}^{j}, \kappa_{0} \neq j,\end{cases} \\
& \mathbf{u}_{n}^{j, \beta}\left(\kappa_{0}\right)= \begin{cases}\left\{E\left[S_{j, \beta}\right] \boldsymbol{\lambda}\right\} \overline{\mathbf{I}}^{j}, & \kappa_{0}=j, \\
\left\{\mathbf{b}_{0}^{j, \beta}\left(\kappa_{0}\right)\left(\mathbf{I}+\mathbf{M}_{1, \beta-1}^{(j, \beta-1)}\right)+E\left[S_{j, \beta}\right] \boldsymbol{\lambda}\right\} \overline{\mathbf{I}}^{j}, \kappa_{0} \neq j,\end{cases}
\end{aligned}
$$

For $\left(\kappa_{0}, a_{0}\right)=(0,0)$,

$$
\begin{aligned}
& \boldsymbol{v}_{g}^{j, \beta}(0,0)=\mathbf{O}, \boldsymbol{v}_{n}^{j, \beta}(0,0)=\mathbf{O}, \mathbf{U}_{g}^{j, \beta}(0)=\mathbf{O}, \mathbf{U}_{n}^{j, \beta}(0)=\mathbf{O}, \\
& \mathbf{u}_{g}^{j, \beta}(0)=E\left[S_{j, \beta}\right] \boldsymbol{\lambda} \mathbf{I}_{1, L_{j}}^{j}, \mathbf{u}_{n}^{j, \beta}(0)=\left\{E\left[S_{j, \beta}\right] \boldsymbol{\lambda}\right\} \overline{\mathbf{I}}^{j} .
\end{aligned}
$$

9.5 The coefficients in Proposition 5

We define the following constants used in Proposition 5.

$$
\boldsymbol{\varphi}_{i, \alpha}(\cdot), \boldsymbol{\eta}_{i, \alpha}(\cdot) \in \mathcal{R}^{2 \times 1} ; \mathbf{w}_{i, \alpha}(\cdot), \mathbf{f}_{i, \alpha}(\cdot) \in \mathcal{R}^{2 J_{c} \times 1} ; w_{i, \alpha}(\cdot), f_{i, \alpha}(\cdot) \in \mathcal{R} .
$$

Let $\hat{\mathbf{w}}_{i, \alpha}(j, \beta, k), \hat{\mathbf{f}}_{i, \alpha}(j, \beta) \in \mathcal{R}^{2 J_{c} \times 1}((i, \alpha),(j, \beta) \in \mathcal{S} ; k \in \Pi)$ be the solutions of the following set of linear equations:

$$
\begin{aligned}
\hat{\mathbf{w}}_{i, \alpha}(j, \beta, k) & =p_{j, \beta, i, \alpha} \mathbf{h}_{00}^{i}(j, k)+\sum_{m=1}^{J} \sum_{\delta=1}^{L_{m}} p_{j, \beta, m, \delta} \mathbf{U}^{m, \delta}(j) \hat{\mathbf{w}}_{i, \alpha}(m, \delta, k), \\
\hat{\mathbf{f}}_{i, \alpha}(j, \beta) & =p_{j, \beta, i, \alpha} \mathbf{f}^{i, \alpha}(j)+\sum_{m=1}^{J} \sum_{\delta=1}^{L_{m}} p_{j, \beta, m, \delta} \mathbf{U}^{m, \delta}(j) \hat{\mathbf{f}}_{i, \alpha}(m, \delta),
\end{aligned}
$$

where $\mathbf{h}_{00}^{i}(j, k), \mathbf{f}^{i, \alpha}(j)$ and $\mathbf{U}^{m, \delta}(j)$ are given in Subsections 9.3 and 9.4. Further let $\hat{w}_{i, \alpha}(j, \beta, k), \hat{f}_{i, \alpha}(j, \beta) \in \mathcal{R}((i, \alpha),(j, \beta) \in \mathcal{S} ; k \in \Pi)$ be the solutions of

$$
\begin{aligned}
\hat{w}_{i, \alpha}(j, \beta, k)= & \sum_{m=1}^{J} \sum_{\delta=1}^{L_{m}} p_{j, \beta, m, \delta} \hat{w}_{i, \alpha}(m, \delta, k) \\
& +\left\{p_{j, \beta, i, \alpha} h_{01}^{i, \alpha}(j, k)+\sum_{m=1}^{J} \sum_{\delta=1}^{L_{m}} p_{j, \beta, m, \delta} \mathbf{u}^{m, \delta}(j) \hat{\mathbf{w}}_{i, \alpha}(m, \delta, k)\right\}, \\
\hat{f}_{i, \alpha}(j, \beta)= & \sum_{m=1}^{J} \sum_{\delta=1}^{L_{m}} p_{j, \beta, m, \delta} \hat{f}_{i, \alpha}(m, \delta) \\
& +\left\{p_{j, \beta, i, \alpha} f^{i, \alpha}(j)+\sum_{m=1}^{J} \sum_{\delta=1}^{L_{m}} p_{j, \beta, m, \delta} \mathbf{u}^{m, \delta}(j) \hat{\mathbf{f}}_{i, \alpha}(m, \delta)\right\},
\end{aligned}
$$

where $h_{01}^{i, \alpha}(j, k), f^{i, \alpha}(j)$ and $\mathbf{u}^{m, \delta}(j)$ are given in Subsections 9.3 and 9.4. Then let us define the following constants used in Proposition 5.

$$
\begin{aligned}
\boldsymbol{\varphi}_{i, \alpha}\left(j, \beta, \kappa_{0}, a_{0}, k\right) & =\mathbf{1}_{i, \alpha}(j, \beta) \boldsymbol{\varphi}^{j}\left(\kappa_{0}, a_{0}, k\right)+\boldsymbol{v}^{j, \beta}\left(\kappa_{0}, a_{0}\right) \hat{\mathbf{w}}_{i, \alpha}(j, \beta, k), \\
\mathbf{w}_{i, \alpha}\left(j, \beta, \kappa_{0}, k\right) & =\mathbf{1}_{i, \alpha}(j, \beta) \mathbf{h}_{00}^{j}\left(\kappa_{0}, k\right)+\mathbf{U}^{j, \beta}\left(\kappa_{0}\right) \hat{\mathbf{w}}_{i, \alpha}(j, \beta, k), \\
w_{i, \alpha}\left(j, \beta, \kappa_{0}, k\right) & =\mathbf{1}_{i, \alpha}(j, \beta) h_{01}^{j, \beta}\left(\kappa_{0}, k\right)+\mathbf{u}^{j, \beta}\left(\kappa_{0}\right) \hat{\mathbf{w}}_{i, \alpha}(j, \beta, k)+\hat{w}_{i, \alpha}(j, \beta, k),
\end{aligned}
$$




$$
\begin{aligned}
\boldsymbol{\eta}_{i, \alpha}\left(j, \beta, \kappa_{0}, a_{0}\right) & =\mathbf{1}_{i, \alpha}(j, \beta) \boldsymbol{\eta}^{j, \beta}\left(\kappa_{0}, a_{0}\right)+\boldsymbol{v}^{j, \beta}\left(\kappa_{0}, a_{0}\right) \hat{\mathbf{f}}_{i, \alpha}(j, \beta), \\
\mathbf{f}_{i, \alpha}\left(j, \beta, \kappa_{0}\right) & =\mathbf{1}_{i, \alpha}(j, \beta) \mathbf{f}^{j, \beta}\left(\kappa_{0}\right)+\mathbf{U}^{j, \beta}\left(\kappa_{0}\right) \hat{\mathbf{f}}_{i, \alpha}(j, \beta), \\
f_{i, \alpha}\left(j, \beta, \kappa_{0}\right) & =\mathbf{1}_{i, \alpha}(j, \beta) f^{j, \beta}\left(\kappa_{0}\right)+\mathbf{u}^{j, \beta}\left(\kappa_{0}\right) \hat{\mathbf{f}}_{i, \alpha}(j, \beta)+\hat{f}_{i, \alpha}(j, \beta),
\end{aligned}
$$

for $(i, \alpha),(j, \beta) \in \mathcal{S} ; \quad\left(\kappa_{0}, a_{0}\right) \in \mathcal{S} \cup\{(0,0)\} ; k \in \Pi$.

9.6 The coefficients in Propositions 6 and 7

We define or calculate the following constants used in Propositions 6 and 7 .

$$
\tilde{q}^{\kappa_{0}, a_{0}}, \tilde{r}^{\kappa_{0}, a_{0}}, \tilde{\varphi}_{i, \alpha}(j, \beta, k), \tilde{\eta}_{i, \alpha}(j, \beta) \in \mathcal{R} .
$$

First we calculate the set of the composite arrival rates $\left\{\Lambda_{i, \alpha}\right\}$ by solving:

$$
\Lambda_{i, \alpha}=\lambda_{i, \alpha}+\sum_{(j, \beta) \in \mathcal{S}} \Lambda_{j, \beta} p_{j, \beta, i, \alpha}, \quad((i, \alpha) \in \mathcal{S}) .
$$

Then we can obtain the following explicit expressions for the time average values defined in (44) and (45).

$$
\begin{aligned}
& \tilde{q}^{\kappa_{0}, a_{0}}= \begin{cases}\Lambda_{\kappa_{0}, a_{0}} E\left[S_{\kappa_{0}, a_{0}}\right], & \left(\kappa_{0}, a_{0}\right) \in \mathcal{S}, \\
1-\rho, & \left(\kappa_{0}, a_{0}\right)=(0,0) .\end{cases} \\
& \tilde{r}^{\kappa_{0}, a_{0}}= \begin{cases}\Lambda_{\kappa_{0}, a_{0}} \overline{s_{\kappa_{0}, a_{0}}^{2}} / 2, & \left(\kappa_{0}, a_{0}\right) \in \mathcal{S}, \\
0, & \left(\kappa_{0}, a_{0}\right)=(0,0) .\end{cases}
\end{aligned}
$$

Further we define the constants which are used in the expressions (49) and (50).

$$
\begin{aligned}
\tilde{\varphi}_{i, \alpha}(j, \beta, k)= & \sum_{\left(\kappa_{0}, a_{0}\right) \in \mathcal{S}}\left(\tilde{r}^{\kappa_{0}, a_{0}}, \tilde{q}^{\kappa_{0}, a_{0}}\right) \boldsymbol{\varphi}_{i, \alpha}\left(j, \beta, \kappa_{0}, a_{0}, k\right) \\
& +\sum_{\left(\kappa_{0}, a_{0}\right) \in \mathcal{S}} \tilde{q}^{\kappa_{0}, a_{0}} w_{i, \alpha}\left(j, \beta, \kappa_{0}, k\right)+(1-\rho) w_{i, \alpha}(j, \beta, 0, k), \\
\tilde{\eta}_{i, \alpha}(j, \beta)= & \sum_{\left(\kappa_{0}, a_{0}\right) \in \mathcal{S}}\left(\tilde{r}^{\kappa_{0}, a_{0}}, \tilde{q}^{\kappa_{0}, a_{0}}\right) \boldsymbol{\eta}_{i, \alpha}\left(j, \beta, \kappa_{0}, a_{0}\right) \\
& +\sum_{\left(\kappa_{0}, a_{0}\right) \in \mathcal{S}} \tilde{q}^{\kappa_{0}, a_{0}} f_{i, \alpha}\left(j, \beta, \kappa_{0}\right)+(1-\rho) f_{i, \alpha}(j, \beta, 0),
\end{aligned}
$$

for $(i, \alpha),(j, \beta) \in \mathcal{S}$ and $k \in \Pi$.

10 Appendix: Algorithm for computing the steady state values and its computational complexity

10.1 The algorithm for computing the steady state values

1. Calculate the following constants used in Proposition 1:

$$
\begin{aligned}
& \mathbf{h}_{10}^{0}(\cdot) \in \mathcal{R}^{2 J_{c} \times 1} ; \boldsymbol{\varphi}^{0}(\cdot) \in \mathcal{R}^{2 \times 1} ; \mathbf{h}_{00}^{0}(\cdot) \in \mathcal{R}^{2 J_{c} \times 1} ; \\
& \mathbf{U}_{1}(\cdot) \in \mathcal{R}^{2 J_{c} \times 2 J_{c}} ; \boldsymbol{v}_{0}(\cdot) \in \mathcal{R}^{2 \times 2 J_{c}} ; \mathbf{U}_{0}(\cdot) \in \mathcal{R}^{2 J_{c} \times 2 J_{c}} ; \mathbf{u}_{0}(\cdot) \in \mathcal{R}^{1 \times 2 J_{c}} .
\end{aligned}
$$

Their detailed expressions are given in Sub-section 9.1. 
2. Calculate the following constants used in Proposition 2:

$$
\mathbf{B}_{1}^{j}(\cdot) \in \mathcal{R}^{2 J_{c} \times J_{c}} ; \mathbf{c}^{j}(\cdot) \in \mathcal{R}^{2 \times J_{c}} ; \mathbf{B}_{0}^{j}(\cdot) \in \mathcal{R}^{2 J_{c} \times J_{c}} ; \mathbf{b}_{0}^{j, \beta}(\cdot) \in \mathcal{R}^{1 \times J_{c}} .
$$

Their detailed expressions are given in Sub-section 9.2.

3. Calculate the following constants used in Proposition 3:

$$
\begin{aligned}
& \mathbf{h}_{10}^{j}(\cdot) \in \mathcal{R}^{2 J_{c} \times 1} ; \\
& \boldsymbol{\varphi}^{j}(\cdot), \boldsymbol{\eta}^{j, \beta}(\cdot) \in \mathcal{R}^{2 \times 1} ; \mathbf{h}_{00}^{j}(\cdot), \mathbf{f}^{j, \beta}(\cdot) \in \mathcal{R}^{2 J_{c} \times 1} ; h_{01}^{j, \beta}(\cdot), f^{j, \beta}(\cdot) \in \mathcal{R} .
\end{aligned}
$$

Their detailed expressions are given in Sub-section 9.3.

4. Calculate the following constants used in Proposition 4:

$$
\boldsymbol{v}^{j, \beta}(\cdot) \in \mathcal{R}^{2 \times 2 J_{c}} ; \mathbf{U}^{j, \beta}(\cdot) \in \mathcal{R}^{2 J_{c} \times 2 J_{c}} ; \mathbf{u}^{j, \beta}(\cdot) \in \mathcal{R}^{1 \times 2 J_{c}} .
$$

Their detailed expressions are given in Sub-section 9.4.

5. Calculate the following constants used in Proposition 5:

$$
\boldsymbol{\varphi}_{i, \alpha}(\cdot), \boldsymbol{\eta}_{i, \alpha}(\cdot) \in \mathcal{R}^{2 \times 1} ; \mathbf{w}_{i, \alpha}(\cdot), \mathbf{f}_{i, \alpha}(\cdot) \in \mathcal{R}^{2 J_{c} \times 1} ; w_{i, \alpha}(\cdot), f_{i, \alpha}(\cdot) \in \mathcal{R} .
$$

Their detailed expressions are given in Sub-section 9.5.

6. Calculate the following constants used in Propositions 6 and 7:

$$
\tilde{q}^{\kappa_{0}, a_{0}}, \tilde{r}^{\kappa_{0}, a_{0}}, \tilde{\varphi}_{i, \alpha}(j, \beta, k), \tilde{\eta}_{i, \alpha}(j, \beta) \in \mathcal{R} .
$$

Their detailed expressions are given in Sub-section 9.6. Then calculate the average numbers of customers:

$$
\tilde{\mathbf{g}}^{k}=\left(\tilde{g}_{i, \alpha}^{k}:(i, \alpha) \in \mathcal{S}\right), \tilde{\mathbf{n}}^{k}=\left(\tilde{n}_{i, \alpha}^{k}:(i, \alpha) \in \mathcal{S}\right) \in \mathcal{R}^{1 \times J_{c}}, \quad(k \in \Pi \cup\{0\})
$$

by solving the following linear equations (Propositions 6 and 7).

$$
\begin{aligned}
& \tilde{n}_{i, \alpha}^{k}=\sum_{(j, \beta) \in \mathcal{S}} \lambda_{j, \beta}\left\{\tilde{\varphi}_{i, \alpha}(j, \beta, k)+\sum_{\kappa_{0} \in \Pi}\left(\tilde{\mathbf{g}}^{\kappa_{0}}, \tilde{\mathbf{n}}^{\kappa_{0}}\right) \mathbf{w}_{i, \alpha}\left(j, \beta, \kappa_{0}, k\right)\right\},(k \in \Pi) ; \\
& \tilde{g}_{i, \alpha}^{i}=\sum_{(j, \beta) \in \mathcal{S}} \lambda_{j, \beta}\left\{\tilde{\eta}_{i, \alpha}(j, \beta)+\sum_{\kappa_{0} \in \Pi}\left(\tilde{\mathbf{g}}^{\kappa_{0}}, \tilde{\mathbf{n}}^{\kappa_{0}}\right) \mathbf{f}_{i, \alpha}\left(j, \beta, \kappa_{0}\right)\right\}-\tilde{q}^{i, \alpha} ; \\
& \tilde{g}_{i, \alpha}^{k}=0,(k \neq i, k \in \Pi) ; \quad \tilde{n}_{i, \alpha}^{0}=0 ; \quad \tilde{g}_{i, \alpha}^{0}=0 .
\end{aligned}
$$

7. Finally we can obtain the average sojourn times (in Proposition 8) by

$$
\begin{aligned}
\bar{w}_{i, \alpha}(j, \beta)= & \left\{\sum_{k \in \Pi} \tilde{\varphi}_{i, \alpha}(j, \beta, k)+\tilde{\eta}_{i, \alpha}(j, \beta)\right\} \\
& +\sum_{\kappa_{0} \in \Pi}\left(\tilde{\mathbf{g}}^{\kappa_{0}}, \tilde{\mathbf{n}}^{\kappa_{0}}\right)\left\{\sum_{k \in \Pi} \mathbf{w}_{i, \alpha}\left(j, \beta, \kappa_{0}, k\right)+\mathbf{f}_{i, \alpha}\left(j, \beta, \kappa_{0}\right)\right\}
\end{aligned}
$$

for $(i, \alpha),(j, \beta) \in \mathcal{S}$. 
10.2 Computational complexity for the steady state values

Let us evaluate the computational complexity for the steady state values. It can be accomplished by evaluating the computational complexity for calculating the constants used in all propositions whose expressions are given in Section 9. For simplicity, we assume that an $n \times n$ matrix can be inverted in $O\left(n^{3}\right)$ operations.

Evaluation of the computational complexity

- Proposition 1: The calculation of the constants in this proposition can be accomplished by simple arithmetic calculations and substitutions of the given constants and the constants related to $\left\{\bar{T}_{i, \alpha}^{\delta}\right\}$ and $\left\{\bar{N}_{i, \alpha, k, \gamma}\right\}$. Hence its computational complexity is at most $O\left(J_{c}^{4}\right)$.

- Proposition 2: The most efforts are required to calculate the set of the matrices $\left\{\mathbf{B}_{1}^{j}\left(\kappa_{0}\right)\right\}$. This calculation requires $J$ inversions of $\left(2 J_{c} J\right) \times\left(2 J_{c} J\right)$ matrices. Hence its computational complexity is at most $O\left(J_{c}^{3} J^{4}\right)$.

- Proposition 3: The most efforts are required to calculate the set of the matrices $\left\{\mathbf{h}_{10}^{j}\left(\kappa_{0}, k\right)\right\}$. This calculation requires $J$ inversions of $\left(2 J_{c} J\right) \times\left(2 J_{c} J\right)$ matrices. Hence its computational complexity is at most $O\left(J_{c}^{3} J^{4}\right)$.

- Proposition 4: The calculation of the constants in this proposition can be accomplished by simple arithmetic calculations, substitutions and multiplications of the matrices obtained previously. Hence its computational complexity is at most $O\left(J_{c}^{4} J\right)$.

- Proposition 5: The most efforts are required to calculate the set of the matrices $\left\{\hat{\mathbf{w}}_{i, \alpha}(j, \beta, k)\right\}$ and $\left\{\hat{\mathbf{f}}_{i, \alpha}(j, \beta)\right\}$. This calculation requires an inversion of $\left(2 J_{c}^{2}\right) \times$ $\left(2 J_{c}^{2}\right)$ matrix. Hence its computational complexity is at most $O\left(J_{c}^{6}\right)$.

- Propositions 6 and 7: The most efforts are required to calculate the set of the average numbers of customers $\left\{\left(\tilde{\mathbf{g}}^{k}, \tilde{\mathbf{n}}^{k}\right)\right\}$. This calculation requires an inversion of $\left(2 J_{c} J\right) \times\left(2 J_{c} J\right)$ matrix. Hence its computational complexity is at most $O\left(J_{c}^{3} J^{3}\right)$.

- Proposition 8: The calculation of the constants in this proposition can be accomplished by simple arithmetic calculations and substitutions of the constants obtained previously. Hence its computational complexity is at most $O\left(J_{c}^{3} J^{2}\right)$.

Hence the overall computational complexity for calculating the steady state values is at most $O\left(J_{c}^{3} J^{4}+J_{c}^{6}\right)$.

\section{Appendix: Polling equations}

In this appendix, we give the generalized definition of the polling equation and the uniqueness of its solution. Let $\mathcal{T}=\{(l, \ell): l=0,1,2, \ldots ; \ell=0,1,2, \ldots\}$ be the index set of pairs of the arrival and the polling instants, and let $\mathcal{V}^{\prime}$ be the set of real valued functions on $\mathcal{S} \times \mathcal{E}_{0} \times\{1,2, \ldots\} \times \mathcal{T}$ where $\mathcal{E}_{0}=\mathcal{E} \backslash\{(0,0,0, \mathbf{0}, \mathbf{0}, L)\}$. We generalize the polling equations (9) and (26).

Polling Equation. Let $f^{0} \in \mathcal{V}^{\prime}$ be any known function. We define a polling equation for an unknown function $f \in \mathcal{V}^{\prime}$ as follows.

$$
\begin{aligned}
f_{j, \beta}(\mathbf{Y}, e, l, \ell)= & f_{j, \beta}^{0}(\mathbf{Y}, e, l, \ell) \\
& +E\left[Z_{P, l, \ell}^{e} f_{j, \beta}\left(\mathbf{Y}\left(\tau_{l, \ell+1}^{e}\right), e, l, \ell+1\right) \mid(j, \beta, \mathbf{Y})_{l, \ell}^{e}, \tau_{l, \ell}^{e}<\tau_{l+1,0}^{e}\right]
\end{aligned}
$$


for $(j, \beta) \in \mathcal{S}, \mathbf{Y}=\left(\kappa_{0}, a_{0}, r, \mathbf{g}, \mathbf{n}, L\right) \in \mathcal{E}_{0}, e \in\{1,2, \ldots\}$ and $(l, \ell) \in \mathcal{T}$ where

$$
Z_{P, l, \ell}^{e}=\left\{\begin{array}{l}
0, M_{l}^{e} \leq \ell \\
1, M_{l}^{e}>\ell
\end{array}\right.
$$

Note. Because $f_{j, \beta}(\mathbf{Y}, e, l, \ell)=H_{j, \beta}^{1}(\mathbf{Y}, e, l, \ell, k)$ and $f_{j, \beta}(\mathbf{Y}, e, l, \ell)=\bar{\nu}_{k, \gamma}^{j, \beta}(\mathbf{Y}, e, l, \ell)$ have the simple explicit expressions for $(l, \ell)=(0,0)$ and $\kappa\left(\tau_{0,0}^{e}-\right)=0$, we have omitted this case from the above equation, that is, the state space is limited to $\mathcal{E}_{0}$.

Then let

$$
\mathcal{V}_{B}^{\prime}=\left\{\begin{array}{c}
\text { There exist nonnegative constants } \mathbf{c}_{f}^{0} \in \mathcal{R}^{2 \times 1}, \\
\mathbf{c}_{f} \in \mathcal{R}^{2 J_{c} \times 1}, c_{f}^{1} \in \mathcal{R} \text { such that } \\
f \in \mathcal{V}^{\prime}: \quad\left|f_{j, \beta}(\mathbf{Y}, e, l, \ell)\right| \leq(r, \mathbf{1}(r)) \mathbf{c}_{f}^{0}+(\mathbf{g}, \mathbf{n}) \mathbf{c}_{f}+c_{f}^{1} \\
\text { for }(j, \beta) \in \mathcal{S}, \mathbf{Y}=\left(\kappa_{0}, a_{0}, r, \mathbf{g}, \mathbf{n}, L\right) \in \mathcal{E}_{0}, \\
e \in\{1,2, \ldots\} \text { and }(l, \ell) \in \mathcal{T} .
\end{array}\right\} .
$$

The objective of this section is to prove the following theorems.

Theorem 1 For any given function $f^{0} \in \mathcal{V}_{B}^{\prime}$, the polling equation (56) has at most one solution on $\mathcal{V}_{B}^{\prime}$.

Theorem 2 The polling equation (26) solved in Proposition 2 has a unique solution for $\bar{\nu}_{k, \gamma}=\bar{\nu}_{k, \gamma}^{j, \beta}(\cdot)$ for all $(k, \gamma) \in \mathcal{S}$. And the polling equation (9) solved in Proposition 3 has a unique solution for $H^{1}(k)=H_{j, \beta}^{1}(\cdot, k)$ for all $k \in \Pi$.

The proof is executed step by step while proving some related lemmas. For convenience, we define

$$
\tilde{\tau}_{l, \ell}^{e}= \begin{cases}\tau_{l, 0}^{e}-, & \ell=0 \\ \tau_{l, \ell}^{e}, & \ell>0\end{cases}
$$

Lemma 1 There exist nonnegative constants $\mathbf{c}_{0 p}^{0} \in \mathcal{R}^{2 \times 2 J_{c}}, \mathbf{C}_{0 p} \in \mathcal{R}^{2 J_{c} \times 2 J_{c}}, \mathbf{c}_{0 p}^{1} \in$ $\mathcal{R}^{1 \times 2 J_{c}}$ such that

$$
E\left[\sum_{k=\ell}^{M_{l}^{e}}\left(\mathbf{g}\left(\tilde{\tau}_{l, k}^{e}\right), \mathbf{n}\left(\tilde{\tau}_{l, k}^{e}\right)\right) \mid(j, \beta, \mathbf{Y})_{l, \ell}^{e}, \tau_{l, \ell}^{e}<\tau_{l+1,0}^{e}\right] \leq(r, \mathbf{1}(r)) \mathbf{c}_{0 p}^{0}+(\mathbf{g}, \mathbf{n}) \mathbf{C}_{0 p}+\mathbf{c}_{0 p}^{1}
$$

for $(j, \beta) \in \mathcal{S}, \mathbf{Y}=\left(\kappa_{0}, a_{0}, r, \mathbf{g}, \mathbf{n}, L\right) \in \mathcal{E}_{0}, e \in\{1,2, \ldots\}$ and $(l, \ell) \in \mathcal{T}$.

Proof: We can show the following equation by using induction and Proposition 1.

$$
\begin{aligned}
& E\left[Z_{P, l, k}^{e}\left(\mathbf{g}\left(\tau_{l, k+1}^{e}\right), \mathbf{n}\left(\tau_{l, k+1}^{e}\right)\right) \mid(j, \beta, \mathbf{Y})_{l, \ell}^{e}, \tau_{l, \ell}^{e}<\tau_{l+1,0}^{e}\right] \\
& =\left\{\begin{array}{cc}
\zeta_{l, \ell}\left(j, \kappa_{0}\right)(\mathbf{g}, \mathbf{n}) \mathbf{R}_{1}\left(\kappa_{0}\right)[\tilde{\mathbf{U}}(j)]^{k-\ell} \mathbf{I}_{0}, & \ell>0, \\
\zeta_{l, \ell}\left(j, \kappa_{0}\right)\left\{(r, \mathbf{1}(r)) \mathbf{\Upsilon}_{0}\left(\kappa_{0}, a_{0}\right)+(\mathbf{g}, \mathbf{n}) \mathbf{R}_{0}\left(\kappa_{0}\right)\right. & \\
\left.+\mathbf{R}_{u}\left(\kappa_{0}, j, \beta\right)\right\}[\tilde{\mathbf{U}}(j)]^{k} \mathbf{I}_{0}, & \ell=0, j \in \mathcal{H}_{e}, \\
\zeta_{l, \ell}\left(j, \kappa_{0}\right)\left\{(r, \mathbf{1}(r)) \mathbf{\Upsilon}_{0}\left(\kappa_{0}, a_{0}\right)+(\mathbf{g}, \mathbf{n}) \mathbf{R}_{0}\left(\kappa_{0}\right)\right. & \\
\left.+\mathbf{R}_{u}\left(\kappa_{0}, j, \beta\right)\right\} \tilde{\mathbf{U}}_{g}(j)[\tilde{\mathbf{U}}(j)]^{k-1} \mathbf{I}_{0}, \ell=0, j \in \mathcal{H}_{g}, k>0, & \\
\zeta_{l, \ell}\left(j, \kappa_{0}\right)\{(r, & \mathbf{1}(r)) \mathbf{\Upsilon}_{0}\left(\kappa_{0}, a_{0}\right)+(\mathbf{g}, \mathbf{n}) \mathbf{R}_{0}\left(\kappa_{0}\right) \\
\left.+\mathbf{R}_{u}\left(\kappa_{0}, j, \beta\right)\right\} \mathbf{I}_{0}, & \ell=0, j \in \mathcal{H}_{g}, k=0,
\end{array}\right.
\end{aligned}
$$


for $k \geq \ell$, where

$$
\begin{aligned}
& \zeta_{l, \ell}\left(j, \kappa_{0}\right)=\left\{\begin{array}{lr}
\mathbf{1}\left\{\kappa_{0} \neq j\right\}, \\
\mathbf{1}\left\{\kappa_{0} \neq j \in \mathcal{H}_{e}\right\}+\mathbf{1}\left\{j \in \mathcal{H}_{g}\right\}, \ell=0
\end{array}\right. \\
& \tilde{\mathbf{U}}(j)=\left(\begin{array}{c}
\tilde{\mathbf{U}}(1, j) \\
\vdots \\
\tilde{\mathbf{U}}(j-1, j) \\
\tilde{\mathbf{O}} \\
\tilde{\mathbf{U}}(j+1, j) \\
\vdots \\
\tilde{\mathbf{U}}(J, j)
\end{array}\right), \quad \tilde{\mathbf{U}}_{g}(j)=\left(\begin{array}{c}
\tilde{\mathbf{U}}(1, j) \\
\vdots \\
\tilde{\mathbf{U}}(j-1, j) \\
\tilde{\mathbf{U}}(j, j) \\
\tilde{\mathbf{U}}(j+1, j) \\
\vdots \\
\tilde{\mathbf{U}}(J, j)
\end{array}\right), \quad \mathbf{I}_{0}=\left(\begin{array}{c}
\mathbf{I} \\
\mathbf{I} \\
\vdots \\
\mathbf{I}
\end{array}\right), \\
& {\left[\begin{array}{l}
\text { where } \tilde{\mathbf{U}}(j), \tilde{\mathbf{U}}_{g}(j) \in \mathcal{R}^{\left(2 J_{c} J\right) \times\left(2 J_{c} J\right)}, \text { and } \mathbf{I}_{0} \in \mathcal{R}^{\left(2 J_{c} J\right) \times\left(2 J_{c}\right)}, \text { and } \\
\tilde{\mathbf{U}}(i, j)=\left(\hat{p}_{i, 1} \mathbf{U}_{1}(1), \ldots, \hat{p}_{i, j-1} \mathbf{U}_{1}(j-1), \mathbf{O}, \hat{p}_{i, j+1} \mathbf{U}_{1}(j+1), \ldots, \hat{p}_{i, J} \mathbf{U}_{1}(J)\right) \\
\in \mathcal{R}^{\left(2 J_{c}\right) \times\left(2 J_{c} J\right)}, \quad(i=1, \ldots, J),
\end{array}\right],} \\
& \mathbf{R}_{1}\left(\kappa_{0}\right)=(\mathbf{O}, \cdots, \mathbf{O}, \underbrace{\mathbf{U}_{1}\left(\kappa_{0}\right)}_{\kappa_{0}^{\text {th }} \text { place }}, \mathbf{O}, \cdots, \mathbf{O}) \in \mathcal{R}^{\left(2 J_{c}\right) \times\left(2 J_{c} J\right)}, \\
& \boldsymbol{\Upsilon}_{0}\left(\kappa_{0}, a_{0}\right)=(\mathbf{0}_{2}, \cdots, \mathbf{0}_{2}, \underbrace{\boldsymbol{v}_{0}\left(\kappa_{0}, a_{0}\right)}_{\kappa_{0}^{\text {th }} \text { place }}, \mathbf{0}_{2}, \cdots, \mathbf{0}_{2}) \in \mathcal{R}^{2 \times\left(2 J_{c} J\right)}, \\
& \mathbf{R}_{0}\left(\kappa_{0}\right)=(\mathbf{O}, \cdots, \mathbf{O}, \underbrace{\mathbf{U}_{0}\left(\kappa_{0}\right)}_{\kappa_{0}^{\text {th }} \text { place }}, \mathbf{O}, \cdots, \mathbf{O}) \in \mathcal{R}^{\left(2 J_{c}\right) \times\left(2 J_{c} J\right)}, \\
& \mathbf{R}_{u}\left(\kappa_{0}, j, \beta\right)=(\mathbf{0}_{1}, \cdots, \mathbf{0}_{1}, \underbrace{\mathbf{u}_{0}\left(\kappa_{0}, j, \beta\right)}_{\kappa_{0}^{\text {th }} \text { place }}, \mathbf{0}_{1}, \cdots, \mathbf{0}_{1}) \in \mathcal{R}^{1 \times\left(2 J_{c} J\right)},
\end{aligned}
$$

where $\tilde{\mathbf{O}} \in \mathcal{R}^{\left(2 J_{c}\right) \times\left(2 J_{c} J\right)}, \mathbf{O} \in \mathcal{R}^{\left(2 J_{c}\right) \times\left(2 J_{c}\right)}, \mathbf{0}_{2} \in \mathcal{R}^{2 \times\left(2 J_{c}\right)}, \mathbf{0}_{1} \in \mathcal{R}^{1 \times\left(2 J_{c}\right)}$ are the zero matrices, and $\mathbf{I} \in \mathcal{R}^{\left(2 J_{c}\right) \times\left(2 J_{c}\right)}$ is the identity matrix.

Hence we have

$$
\begin{aligned}
E & {\left[\sum_{k=\ell+1}^{M_{l}^{e}}\left(\mathbf{g}\left(\tau_{l, k}^{e}\right), \mathbf{n}\left(\tau_{l, k}^{e}\right)\right) \mid \begin{array}{c}
(j, \beta, \mathbf{Y})_{l, \ell}^{e} \\
\tau_{l, \ell}^{e}<\tau_{l+1,0}^{e}
\end{array}\right] } \\
= & \sum_{k=\ell}^{\infty} E\left[Z_{P, l, k}^{e}\left(\mathbf{g}\left(\tau_{l, k+1}^{e}\right), \mathbf{n}\left(\tau_{l, k+1}^{e}\right)\right) \mid \begin{array}{c}
(j, \beta, \mathbf{Y})_{l, \ell}^{e} \\
\tau_{l, \ell}^{e}<\tau_{l+1,0}^{e}
\end{array}\right] \\
= & \left\{\begin{array}{cc}
\zeta_{l, \ell}\left(j, \kappa_{0}\right)(\mathbf{g}, \mathbf{n}) \mathbf{R}_{1}\left(\kappa_{0}\right)[\tilde{\mathbf{I}}-\tilde{\mathbf{U}}(j)]^{-1} \mathbf{I}_{0}, & \ell>0, \\
\zeta_{l, \ell}\left(j, \kappa_{0}\right)\left\{(r, \mathbf{1}(r)) \mathbf{\Upsilon}_{0}\left(\kappa_{0}, a_{0}\right)+(\mathbf{g}, \mathbf{n}) \mathbf{R}_{0}\left(\kappa_{0}\right)\right. & \ell=0, j \in \mathcal{H}_{e}, \\
\left.+\mathbf{R}_{u}\left(\kappa_{0}, j, \beta\right)\right\}[\tilde{\mathbf{I}}-\tilde{\mathbf{U}}(j)]^{-1} \mathbf{I}_{0}, & \\
\zeta_{l, \ell}\left(j, \kappa_{0}\right)\left\{(r, \mathbf{1}(r)) \mathbf{\Upsilon}_{0}\left(\kappa_{0}, a_{0}\right)+(\mathbf{g}, \mathbf{n}) \mathbf{R}_{0}\left(\kappa_{0}\right)\right. & \\
\left.+\mathbf{R}_{u}\left(\kappa_{0}, j, \beta\right)\right\}\left\{\tilde{\mathbf{I}}+\tilde{\mathbf{U}} g(j)[\tilde{\mathbf{I}}-\tilde{\mathbf{U}}(j)]^{-1}\right\} \mathbf{I}_{0}, \ell=0, j \in \mathcal{H}_{g},
\end{array}\right.
\end{aligned}
$$

where $\tilde{\mathbf{I}} \in \mathcal{R}^{\left(2 J_{c} J\right) \times\left(2 J_{c} J\right)}$ is the identity matrix. The last equality comes from the fact that $[\tilde{\mathbf{U}}(j)]^{k} \rightarrow \mathbf{O} \in \mathcal{R}^{\left(2 J_{c} J\right) \times\left(2 J_{c} J\right)}$ as $k \rightarrow \infty$. This completes the proof of this lemma. 
Lemma 2 Suppose that $f \in \mathcal{V}_{B}^{\prime}$ satisfies the following inequality:

$$
\left|f_{j, \beta}(\mathbf{Y}, e, l, \ell)\right| \leq E\left[Z_{P, l, \ell}^{e}\left|f_{j, \beta}\left(\mathbf{Y}\left(\tau_{l, \ell+1}^{e}\right), e, l, \ell+1\right)\right| \mid(j, \beta, \mathbf{Y})_{l, \ell}^{e}, \tau_{l, \ell}^{e}<\tau_{l+1,0}^{e}\right]
$$

for $(j, \beta) \in \mathcal{S}, \mathbf{Y}=\left(\kappa_{0}, a_{0}, r, \mathbf{g}, \mathbf{n}, L\right) \in \mathcal{E}_{0}, e \in\{1,2, \ldots\}$ and $(l, \ell) \in \mathcal{T}$. Then

$$
f=0 \text {. }
$$

Proof: By recursively applying the condition of this lemma, we have

$$
\begin{aligned}
& \left|f_{j, \beta}(\mathbf{Y}, e, l, \ell)\right| \\
& \quad \leq E\left[Z_{P, l, \ell+k}^{e}\left|f_{j, \beta}\left(\mathbf{Y}\left(\tau_{l, \ell+k+1}^{e}\right), e, l, \ell+k+1\right)\right| \mid(j, \beta, \mathbf{Y})_{l, \ell}^{e}, \tau_{l, \ell}^{e}<\tau_{l+1,0}^{e}\right]
\end{aligned}
$$

for $k=0,1,2, \ldots$. Because $f \in \mathcal{V}_{B}^{\prime}$, there exist nonnegative constants $\mathbf{c}_{f}^{0} \in \mathcal{R}^{2 \times 1}$, $\mathbf{c}_{f} \in \mathcal{R}^{2 J_{c} \times 1}, c_{f}^{1} \in \mathcal{R}$ such that

$$
\begin{aligned}
\left|f_{j, \beta}(\mathbf{Y}, e, l, \ell)\right| \leq E\left[Z _ { P , l , \ell + k } ^ { e } \left\{\left(r\left(\tau_{l, \ell+k+1}^{e}\right), \mathbf{1}\left(r\left(\tau_{l, \ell+k+1}^{e}\right)\right)\right) \mathbf{c}_{f}^{0}\right.\right. \\
\left.\left.\quad+\left(\mathbf{g}\left(\tau_{l, \ell+k+1}^{e}\right), \mathbf{n}\left(\tau_{l, \ell+k+1}^{e}\right)\right) \mathbf{c}_{f}+c_{f}^{1}\right\} \mid(j, \beta, \mathbf{Y})_{l, \ell}^{e}, \tau_{l, \ell}^{e}<\tau_{l+1,0}^{e}\right] \\
=E\left[Z_{P, l, \ell+k}^{e}\left(\mathbf{g}\left(\tau_{l, \ell+k+1}^{e}\right), \mathbf{n}\left(\tau_{l, \ell+k+1}^{e}\right)\right) \mid(j, \beta, \mathbf{Y})_{l, \ell}^{e}, \tau_{l, \ell}^{e}<\tau_{l+1,0}^{e}\right] \mathbf{c}_{f} \\
\quad \quad+E\left[Z_{P, l, \ell+k}^{e} \mid(j, \beta, \mathbf{Y})_{l, \ell}^{e}, \tau_{l, \ell}^{e}<\tau_{l+1,0}^{e}\right] c_{f}^{1} \\
\rightarrow 0, \quad(k \rightarrow \infty) .
\end{aligned}
$$

This expression converges to 0 because

$$
\begin{aligned}
& \sum_{k=\ell}^{\infty} E\left[Z_{P, l, k}^{e}\left(\mathbf{g}\left(\tilde{\tau}_{l, k+1}^{e}\right), \mathbf{n}\left(\tilde{\tau}_{l, k+1}^{e}\right)\right) \mid(j, \beta, \mathbf{Y})_{l, \ell}^{e}, \tau_{l, \ell}^{e}<\tau_{l+1,0}^{e}\right] \\
& =E\left[\sum_{k=\ell}^{M_{l}^{e}-1}\left(\mathbf{g}\left(\tilde{\tau}_{l, k+1}^{e}\right), \mathbf{n}\left(\tilde{\tau}_{l, k+1}^{e}\right)\right) \mid(j, \beta, \mathbf{Y})_{l, \ell}^{e}, \tau_{l, \ell}^{e}<\tau_{l+1,0}^{e}\right]<\infty, \\
& E\left[Z_{P, l, \ell+k}^{e} \mid(j, \beta, \mathbf{Y})_{l, \ell}^{e}, \tau_{l, \ell}^{e}<\tau_{l+1,0}^{e}\right]=P\left\{M_{l}^{e}>\ell+k \mid(j, \beta, \mathbf{Y})_{l, \ell}^{e}, \tau_{l, \ell}^{e}<\tau_{l+1,0}^{e}\right\} \\
& =\left\{\begin{array}{lr}
P\left\{\kappa\left(\tau_{l, \ell+1}^{e}\right) \neq j, \ldots, \kappa\left(\tau_{l, \ell+k}^{e}\right) \neq j \mid \kappa\left(\tilde{\tau}_{l, \ell}^{e}\right)=\kappa_{0}\right\}, & \zeta_{l, \ell}\left(j, \kappa_{0}\right)=1, \\
0, & \zeta_{l, \ell}\left(j, \kappa_{0}\right)=0
\end{array}\right. \\
& \rightarrow 0, \quad(k \rightarrow \infty) \text {. }
\end{aligned}
$$

The last expression comes from the fact that the (finite state) Markov chain generated by the transition probability matrix $\hat{\mathbf{P}}$ is irreducible.

Proof of Theorem 1: Let $f \in \mathcal{V}_{B}^{\prime}$ and $\hat{f} \in \mathcal{V}_{B}^{\prime}$ be any two solutions that satisfy the equation (56) for the given $f^{0}$. Then

$$
\begin{aligned}
& \left|f_{j, \beta}(\mathbf{Y}, e, l, \ell)-\hat{f}_{j, \beta}(\mathbf{Y}, e, l, \ell)\right| \\
& \leq E\left[Z_{P, l, \ell}^{e}\left|f_{j, \beta}\left(\mathbf{Y}\left(\tau_{l, \ell+1}^{e}\right), e, l, \ell+1\right)-\hat{f}_{j, \beta}\left(\mathbf{Y}\left(\tau_{l, \ell+1}^{e}\right), e, l, \ell+1\right)\right| \mid \begin{array}{c}
(j, \beta, \mathbf{Y})_{l, \ell}^{e} \\
\tau_{l, \ell}^{e}<\tau_{l+1,0}^{e}
\end{array}\right]
\end{aligned}
$$

for $(j, \beta) \in \mathcal{S}, \mathbf{Y} \in \mathcal{E}_{0}, e \in\{1,2, \ldots\}$ and $(l, \ell) \in \mathcal{T}$. Since $f-\hat{f} \in \mathcal{V}_{B}^{\prime}$, we have from Lemma $2, f=\hat{f}$.

We next show that the quantities $\bar{\nu}_{k, \gamma}=\bar{\nu}_{k, \gamma}^{j, \beta}(\cdot)$ for all $(k, \gamma) \in \mathcal{S}$ and $H^{1}(k)=$ $H_{j, \beta}^{1}(\cdot, k)$ for all $k \in \Pi$ respectively defined in Sections 2 and 4 are indeed the functions in $\mathcal{V}_{B}^{\prime}$. 
Lemma 3 Let $\bar{\nu}_{k, \gamma}=\bar{\nu}_{k, \gamma}^{j, \beta}(\cdot)$ be the number of $(k, \gamma)$-customers at time $\tau_{l, M_{l}^{e}}^{e}$ defined in (23), and let $H^{1}(k)=H_{j, \beta}^{1}(\cdot, k)$ be the performance measures defined in (6). Then, $\bar{\nu}_{k, \gamma} \in \mathcal{V}_{B}^{\prime}$ for all $(k, \gamma) \in \mathcal{S}$, and $H^{1}(k) \in \mathcal{V}_{B}^{\prime}$ for all $k \in \Pi$.

Proof: $\bar{\nu}_{k, \gamma} \in \mathcal{V}_{B}^{\prime}$ can be easily shown by Lemma 1 .

Now we consider $H^{1}(k) \geq 0$.

$$
\begin{aligned}
& H_{j, \beta}^{1}(\mathbf{Y}, e, l, \ell, k)=E\left[\int_{\tau_{l, \ell}^{e}}^{\tau_{l+1,0}^{e}} C_{W j, \beta}^{e}(t) \mathbf{1}\{\kappa(t)=k\} d t \mid \begin{array}{l}
(j, \beta, \mathbf{Y})_{l, \ell}^{e} \\
\tau_{l, \ell}^{e}<\tau_{l+1,0}^{e}
\end{array}\right]
\end{aligned}
$$

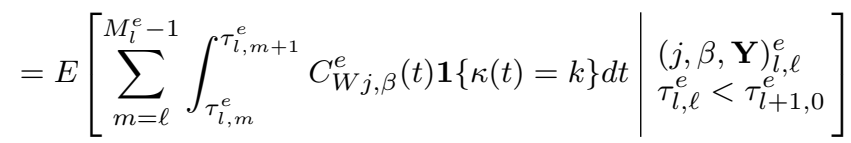

$$
\begin{aligned}
& =\sum_{m=\ell}^{\infty} E\left[Z_{P, l, m}^{e} \int_{\tau_{l, m}^{e}}^{\tau_{l, m+1}^{e}} C_{W j, \beta}^{e}(t) \mathbf{1}\{\kappa(t)=k\} d t \mid \begin{array}{c}
(j, \beta, \mathbf{Y})_{l, \ell}^{e} \\
\tau_{l, \ell}^{e}<\tau_{l+1,0}^{e}
\end{array}\right] \\
& =\sum_{m=\ell}^{\infty} E\left[\begin{array}{l|l}
Z_{P, l, m}^{e} H_{j, \beta}^{0}\left(\mathbf{Y}\left(\tilde{\tau}_{l, m}^{e}\right), e, l, m, k\right) & \begin{array}{l}
(j, \beta, \mathbf{Y})_{l, \ell}^{e} \\
\tau_{l, \ell}^{e}<\tau_{l+1,0}^{e}
\end{array}
\end{array}\right] \\
& \leq \sum_{m=\ell}^{\infty} E\left[Z_{P, l, m}^{e}\left\{\left(r\left(\tilde{\tau}_{l, m}^{e}\right), \mathbf{1}\left(r\left(\tilde{\tau}_{l, m}^{e}\right)\right)\right) \boldsymbol{\varphi}^{0}+\left(\mathbf{g}\left(\tilde{\tau}_{l, m}^{e}\right), \mathbf{n}\left(\tilde{\tau}_{l, m}^{e}\right)\right) \mathbf{h}^{0}\right\} \mid \begin{array}{l}
(j, \beta, \mathbf{Y})_{l, \ell}^{e} \\
\tau_{l, \ell}^{e}<\tau_{l+1,0}^{e}
\end{array}\right] \\
& =E\left[\sum_{m=\ell}^{M_{l}^{e}-1}\left\{\left(r\left(\tilde{\tau}_{l, m}^{e}\right), \mathbf{1}\left(r\left(\tilde{\tau}_{l, m}^{e}\right)\right)\right) \varphi^{0}+\left(\mathbf{g}\left(\tilde{\tau}_{l, m}^{e}\right), \mathbf{n}\left(\tilde{\tau}_{l, m}^{e}\right)\right) \mathbf{h}^{0}\right\} \mid \begin{array}{l}
(j, \beta, \mathbf{Y})_{l, \ell}^{e} \\
\tau_{l, \ell}^{e}<\tau_{l+1,0}^{e}
\end{array}\right] \\
& \leq(r, \mathbf{1}(r)) \boldsymbol{\varphi}^{0}+\left\{(r, \mathbf{1}(r)) \mathbf{c}_{0 p}^{0}+(\mathbf{g}, \mathbf{n}) \mathbf{C}_{0 p}+\mathbf{c}_{0 p}^{1}\right\} \mathbf{h}^{0}
\end{aligned}
$$

where $\varphi^{0}$ and $\mathbf{h}^{0}$ are the constants that respectively satisfy

$$
\varphi^{0}\left(\kappa_{0}, a_{0}, j, k\right) \leq \varphi^{0} \text { and } \mathbf{h}_{10}^{0}\left(\kappa_{0}, j, k\right), \mathbf{h}_{00}^{0}\left(\kappa_{0}, j, k\right) \leq \mathbf{h}^{0}
$$

The first inequality comes from Proposition 1 and the second inequality comes from Lemma 1.

Proof of Theorem 2: The function $\bar{\nu}_{k, \gamma}=\bar{\nu}_{k, \gamma}^{j, \beta}(\cdot)$ defined in (23) is an element of $\mathcal{V}_{B}^{\prime}$ for any $(k, \gamma) \in \mathcal{S}$ from Lemma 3 and satisfies the polling equation given given by (26). Hence from Theorem $1 \bar{\nu}_{k, \gamma}$ given by (25) is the unique solution of the polling equation. (The function $f^{0}$ related to $\bar{\nu}_{k, \gamma}$ is obviously in $\mathcal{V}_{B}^{\prime}$.)

The function $H^{1}(k)=H_{j, \beta}^{1}(\cdot, k)$ defined in (6) is an element of $\mathcal{V}_{B}^{\prime}$ for any $k \in \Pi$ from Lemma 3 and satisfies the polling equation given by (9). Hence from Theorem $1 H^{1}(k)$ given by $(27)$ is the unique solution of the polling equation. (The function $f^{0}=H_{j, \beta}^{0}(\cdot, k)$ defined in $(7)$ is in $\mathcal{V}_{B}^{\prime}$ from Proposition 1.)

Acknowledgements The author would thank the reviewers for their valuable comments which helped to improve the paper. 


\section{References}

Aalto, S., Ayesta, U., Borst, S., Misra, V. \& Núñez-Queija, R. (2007). Beyond processor sharing. ACM SIGMETRICS Performance Evaluation Review, 34, 36-43.

Altman, E., Avrachenkov, K. \& Ayesta, U. (2006). A survey on discriminatory processor sharing. Queueing Systems, 53, 53-63.

Baker, J. E., \& Rubin, I. (1987). Polling with a general-service order table. IEEE Transactions on Communications, 35, 283-288.

Boon, M. A. A., Adan, I. J. B. F. \& Boxma, O. J. (2010). A polling model with multiple priority levels. Performance Evaluation, 67, 468-484.

Boxma, O. J., Bruin, J. \& Fralix, B. (2009). Sojourn times in polling systems with various service disciplines. Performance Evaluation, 66, 621-639.

Boxma, O. J., Levy, H. \& Yechiali, U. (1992). Cyclic reservation schemes for efficient operation of multiple-queue single-server systems. Annals of Operations Research, 35, 187-208.

Choi, B. D., Kim, B., \& Choi, S. H. (2000). On the M/G/1 Bernoulli feedback queue with multi-class customers. Computers \& Operations Research, 27, 269-286.

Cooper, R. B. (1970). Queues served in cyclic order: Waiting times. The Bell System Technical Journal, 49, 399-413.

Cooper, R. B., \& Murray, G. (1969). Queues served in cyclic order. The Bell System Technical Journal, 48, 675-689.

Doshi, B. T., \& Kaufman, J. S. (1988). Sojourn time in an M/G/1 queue with Bernoulli feedback. In O. J. Boxma, \& R. Syski (Eds.), Queueing theory and its applications (pp. 207-233). Amsterdam: North-Holland.

Eisenberg, M. (1972). Queues with periodic service and changeover time. Operations Research, 20, 440-451.

Ferguson, M. J., \& Aminetzah, Y. J. (1985). Exact results for nonsymmetric token ring systems IEEE Transactions on Communications, 33, 223-231.

Hirayama, T. (2003). Mean sojourn times in multiclass feedback queues with gated disciplines. Naval Research Logistics, 50, 719-741.

Hirayama, T. (2005). Multiclass polling systems with Markovian feedback: Mean sojourn times in gated and exhaustive systems with local priority and FCFS service orders. Journal of the Operations Research Society of Japan, 48, 226-255.

Hirayama, T. (2009a). Markovian polling systems: Functional computation for mean waiting times and its computational complexity. In W. Yue, Y. Takahashi, \& H. Takagi (Eds.), Advances in queueing theory and network applications (pp. 119-146). New York: Springer.

Hirayama, T. (2009b). Multi-layered scheduling in a Markovian polling system. In Proceedings of the queueing symposium: Stochastic models and their applications, Matsumoto, Japan 21-23, January 2009, (pp. 258-267).

Hirayama, T. (2010). Analysis of multiclass feedback queues and its application to a packet scheduling problem. Journal of Industrial and Management Optimization, 6, 541-568.

Hirayama, T., Hong, S. J., \& Krunz, M. M. (2004). A new approach to analysis of polling systems. Queueing Systems, 48, 135-158.

Jewkes, E. M., \& Buzacott, J. A. (1991). Flow time distributions in a $K$ class M/G/1 priority feedback queue. Queueing Systems, 8, 183-202.

Katayama, T. (1992). Performance analysis and optimization of a cyclic-service tandem queueing system with multi-class customers. Computers 8 Mathematics with Applications, 24, $25-33$

Kleinrock, L. (1976). Queueing systems, volume II: Computer applications. New York: Wiley.

Kleinrock, L., \& Levy, H. (1988). The analysis of random polling systems. Operations Research, $36,716-732$

Konheim, A. G., Levy, H., \& Srinivasan, M. M. (1994). Descendant set: An efficient approach for the analysis of polling systems. IEEE Transactions on Communications, 42, 1245-1253.

Levy, H. (1991). Binomial-gated service: A method for effective operation and optimization of polling systems. IEEE Transactions on Communications, 39, 1341-1350.

Levy, H., \& Sidi, M. (1990). Polling systems: Applications, modeling, and optimization. IEEE Transactions on Communications, 38, 1750-1760.

Paterok, M., Fischer, O., \& Opta, L. (1989). Feedback queues with preemption-distance priorities. Performance Evaluation Review, 17, 136-145.

Resing, J, A. C. (1993). Polling systems and multitype branching processes. Queueing Systems, 13, 409-426. 
Sarkar, D. \& Zangwill, W. I. (1989). Expected waiting time for nonsymmetric cyclic queueing systems-Exact results and applications. Management Science, 35, 1463-1474.

Sidi, M., Levy, H., \& Fuhrmann, S. W. (1992). A queueing network with a single cyclically roving server. Queueing Systems, 11, 121-144.

Simon, B. (1984). Priority queues with feedback. Journal of the ACM, 31, 134-149.

Srinivasan, M. M. (1991). Nondeterministic polling systems. Management Science, 37, 667681.

Takagi, H. (1986). Analysis of polling systems. Cambridge: The MIT Press.

Takine, T., Takagi, H., \& Hasegawa, T. (1991). Sojourn times in vacation and polling systems with Bernoulli feedback. Journal of Applied Probability, 28, 422-432.

Van den Berg, J. L., Boxma, O. J., \& Groenendijk, W. P. (1989). Sojourn times in the M/G/1 queue with deterministic feedback. Commun. Statist.-Stochastic Models, 5(1), 115-129.

Van der Mei, R. D. (2002). Waiting-time distributions in polling systems with simultaneous batch arrivals. Annals of Operations Research, 113, 155-173.

Wierman, A., Winands, E. M. M., \& Boxma, O. J. (2007). Scheduling in polling systems. Performance Evaluation, 64, 1009-1028.

Winands, E. M. M., Adan, I. J. B. F., \& Van Houtum, G. J. (2006). Mean value analysis for polling systems. Queueing Systems, 54, 35-44. 